MANN SB 411

N27 N3 FIONAL 1921 E SOCIETY.

\title{
Select List
}

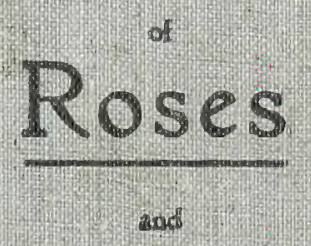

Instructions

for Pruning. 


\section{ALBERT R. MANN}

LIBRARY

AT

CORNELL UNIVERSITY

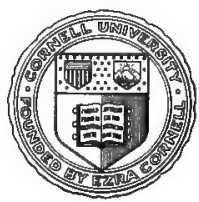

THE GIFT OF

Prof. Carl F. Gortzig

Floriculture \& Ornamental Horticulture 


\section{Cornell University Library}

The original of this book is in the Cornell University Library.

There are no known copyright restrictions in the United States on the use of the text.

http://www.archive.org/details/cu31924089568020 




\section{PUBLICATIONS}

\section{of $\mathrm{Tux}$ \\ National Rose Society.}

Issued only to Members.

"Select List of Roses and Instructions for Pruning."

New and revised edition. Illustrated and bound in cloth.

This list contains descriptions of the best Roses in cultivation, and also the purposes for which each variety is best suited, together with instructions for pruning.

"The Enemies of the Rose." Illustrated in colours and bound in cloth. This handbook on the Fungoid and Insect Pests of the Rose supplies a want long felt by all classes of Rosarians.

"Hints on Planting Roses." In this edition is inserted a selection of 40 choice "Exhibition" Roses, and 80 Non-exhibition or "Decorative" Roses, suitable for beginning a collection.

"The Rose Annual." This is issued early in each year, and contains articles and illustrations of interest to all classes of Rosarians.

Copies of the above Publications can be obtained direct from-

COURTNEY PAGE, Hon. Secretary,

25, Victoria StrRet, Westminster, S.W.

Telephone: Victoria 959.

National Rose Sucietr.-All interested in Rose Culture are invited to join this Society. There is no ballot for membership. The Annual Subscription is $£$ I Is. or 10/6. All the above Publications are siupplied to Nere Members free of charge. Application for membership should be made to the Hon. Secretary. 25. Victoria Street, Westminster, S.W. New Members also receive tickets for the Royal Botanic and Autumn Shows. 


\title{
The National Rose Society's
}

\author{
SELECT
}

$$
\text { LIST OF ROSES, }
$$

AND

\section{INSTRUCTIONS FOR}

\section{PRUNING.}

$* 2$

Compiled by the Publications Committee of the Society.

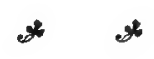
$-1921$.

[Copyright by Courtiey Page, Hon. Secretary, 25, Victoria Street, London, S.W.? 



\section{PREFACE.}

THIS edition of the Select List of Roses and Instructions for Pruning has been prepared by the Publications Committee of the N.R.S., generally on the lines of the previous edition of 1917 .

The List of Roses is a selection and not a catalogue. The number of varieties of Roses increases rapidly every year, and the average rose lover cannot grow more than a very small proportion of the thousands of varieties available.

'The Publications Committee have included in this volume those varieties which, as the result of experience in many parts of the country, they consider may, with fair treatment and under average conditions, be relied on for the purposes for which they are recommended. The List is still a long one. Many Roses have been removed from the previous List and new ones have been added, to bring the List up to date. Each excision and addition has been carefully considered by the Publications Committee and determined by a majority of votes.

The Committee do not suggest that Rose growers of experience or special local knowledge should restrict themselves to the Roses included in this volume. There are many varieties not mentioned here, which, under special local conditions, in soils particularly adapted to their growth, and under other favourable circumstances, will prove useful in the garden, and if any Rose grower finds that some varisty not included 
gives satisfactory results under his particular conditions, he should be slow to discard it in favour of any other variety, however desirable, which has been included.

No Rose has been included in the general list which has not been grown by some member of the Committee, but information as to the newer Roses is of necessity imperfect, and judgment as to these must be considered provisional.

Roses of I920 which appear promising have been included in a Special List, but the Committee do not profess to pass any final opinion upon them.

The section as to pruning has been revised in the light of the further experience gained since the publication of the last edition. The descriptions appearing in the Select List from pages 29 to 71 end with a reference to a figure, e.g., "Anna Olivier-prune 4 or 5 ," and this indication is intended to refer to the relative number of the instruction contained in the pruning section on pages 83 to 121 .

In the case of some of the more vigorous dwarf Roses these pruning instructions may, under suitable circumstances, be wholly or partially disregarded. Where ample space can be allowed, and under congenial conditions, many of these Roses will grow naturally into large bushes, and under such conditions they will thrive best with very little pruning beyond the removal of old wood, and will produce in their season a wealth of bloom which cannot be equalled by plants of the same variety which have been pruned in the more orthodox fashion. 


\section{TABLE OF CONTENTS.}

\section{OFFICIAL LIST OF ROSES.}

$\begin{array}{llllllll} & & & & & & & \\ \text { PREFACE } & \cdots & \cdots & \ldots & \ldots & \ldots & \ldots & 3\end{array}$

$\begin{array}{lllllllll}\text { INDEX.. } & \ldots & \ldots & \ldots & \ldots & \ldots & \ldots & 7\end{array}$

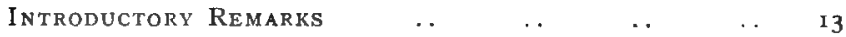

Selection of Roses for the Various Purposes, viz:-

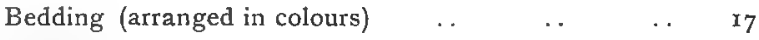

$\begin{array}{lllllll}\text { Standards } & \ldots & \ldots & \ldots & \ldots & \ldots & \text { I9 }\end{array}$

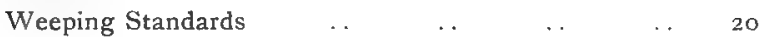

$\begin{array}{llllllll}\text { Walls } & . & & \ldots & \ldots & \ldots & \ldots & \end{array}$

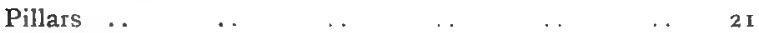

$\begin{array}{lllllll}\text { Covering Banks .. } & \ldots & \ldots & \ldots & \ldots & 22\end{array}$

$\begin{array}{lllllll}\text { Pegging Down } & \ldots & \ldots & \ldots & \ldots & & \\ 22\end{array}$

$\begin{array}{llllll}\text { Forming a Hedge } & \ldots & \ldots & \ldots & \ldots & \mathbf{2 2}\end{array}$

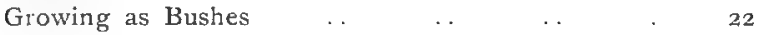

$\begin{array}{llllllll}\text { Towns } & . . & \ldots & \ldots & \ldots & \ldots & \ldots & 23\end{array}$

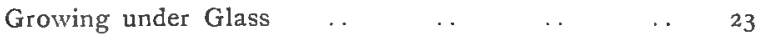

$\begin{array}{lllllll}\text { Fragrant Roses .. } & \ldots & \ldots & \ldots & \ldots & 24\end{array}$

$\begin{array}{llllll}\text { Single-flowered Roses } & \ldots & \ldots & \ldots & \ldots & 25\end{array}$

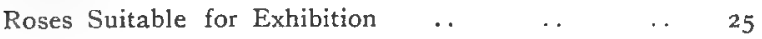

Roses Suitable for Exhibition as Decoratives $\quad . \quad 26$

$\begin{array}{llllll}\text { Penzance Briars .. } & \ldots & \ldots & \ldots & \ldots & 27\end{array}$

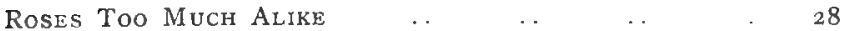

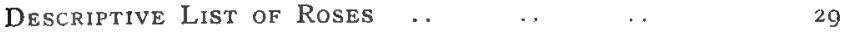

A Descriptive List of Some Recent Roses .. . . 70

Roses in Their Respective Classes .. 
TABLE OF CONTENTS (continued)-

INSTRUCTIONS FOR PRUNING.

Climbing Roses under Glass

Early Autumn Thinning

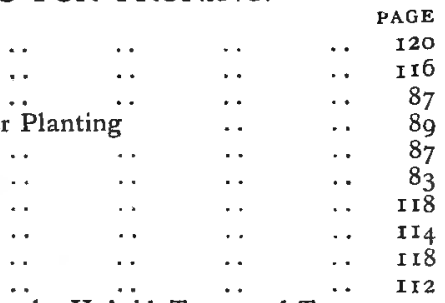

$\begin{array}{llll}\text { How to use the Instructions } & & \ldots & \\ \text { How to Prune the first time after Planting } & \ldots & \ldots & 87\end{array}$

Instruments for Pruning

Pegged-down Roses

Roses for Pergolas and Piliars

Roses in Pots under Glass

Standard Roses

I I 2

Time of Pruning Hybrid Perpetuals, Hybrid Teas, and Teas and Noisettes

$\because$

PRUNING ROSES FOR EXHIBITION PURPOSES.

Hybrid Perpetuals', Hybrid Teas, and Teas and Noisettes, 9 I, 93, 95, 97 PRUNING ROSES FOR GARDEN PURPOSES.

Alba ..

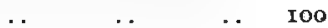

Austrian Briar ..

Ayrshire

Banksian

Bourbon

Boursault

China

Dijon Tea

French and Damask

.. $\quad$.. IOI

Hybrid Bourbon

Hybrid Bracteata

Hybrid China ..

Hybrid Musk ..

Hybrid Noisette

Hybrid Perpetual

Hybrid Tea

Hybrids of Species

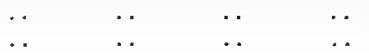

- IOI

Miniature Provence

$\begin{array}{lll}\cdots & \cdots & 103 \\ \cdots & \cdots & 104 \\ \cdots & \cdots & 108\end{array}$

Moss ..

Multiflora rambler

Noisette

Pernetiana or Austrian Hybrid

Perpetual Scotch

Polyantha (Pompon)

Provence

Rugosa

Scotch Briar

Sempervirens

Species

Sweet Briar and its Hybrids

Tea

Wichuraiana Rambler

Wichuraiana Pompon

$\cdots$

.

$\begin{array}{lll}. & \ldots & 108\end{array}$

$\begin{array}{lll}\cdots & \cdots & \text { 108 } \\ \cdots & \cdots & \text { 1 } 10\end{array}$

.. $\quad$.. 100

. $\quad . \quad$ IOI

$\because \quad \ldots$ I I I

$\cdots \quad \cdots \quad$ 100

$\begin{array}{lll}\cdots & \cdots & 100 \\ \cdots & \cdots & \text { roI }\end{array}$

.. $\quad$.. $\quad$ IOI

.. $\quad 90,92,94$

.. $90,92,94,96$

.. $\quad$.. In

. $\quad$.. 99

$\begin{array}{lll}\cdots & . & 99 \\ \ldots & . . & 99\end{array}$

$\begin{array}{llr}\ldots & \ldots & 99 \\ . & . . & \text { ro4 }\end{array}$

$\begin{array}{lll}\cdots & \ldots & 104 \\ . & \ldots & \text { 109 }\end{array}$

$\begin{array}{lll}\ldots & \ldots & \text { rog } \\ \ldots & . . & \text { ro2 }\end{array}$

$\begin{array}{lll}\cdots & \ldots & \text { IOZ } \\ \ldots & . . & \text { III }\end{array}$

$\begin{array}{lll}\cdots & \cdots & \text { II } \\ \cdots & \ldots & \text { I }\end{array}$

. $\quad$. $\quad 99$

.. $\quad$. $\quad$ I IO

$\begin{array}{lll}. & \ldots & 1102\end{array}$

. $\quad$. $\quad$ ro3

$\begin{array}{lll}\ldots & \ldots & 103\end{array}$

.. $\quad$.. IO3

.. $90,92,94,96$

.. $\quad . . \quad$ I I I

$\begin{array}{llllll}\text {. } & \text {. } & \ldots & \ldots & \ldots & \text { II } 2\end{array}$ 


\section{INDEX.}

PAGE

PAGE

Adimiral Ward ........... 18, 29, 74

Ennchen Müller .............. 29, 77

Aglaia ......................... 29, 76

Aimée Vibert, Climbing ... 29, 76

Albérie Barbier 20, 21, 22, 26, 29, 78

A.lexander Hrill Gray ... 19, 23,

$25,30,78$

Alexander Elmslie ........... 29, 74

Alfred Colomb ...................... 28

Alister Stella Gray 19, 21, 30, 76 alpina .......................... 30, 7

alpina pyrenaica ............. 30, it

altaica $\ldots . . . . . . . \ldots . . .22,25,30$, it

American Pillar 21, 24, 25, 26, 30, 78 Amy Robsart 27,30 , 78 Andersoni $25,30,7 i$ Anna Olivier .......... 19, 30, is Anne of Geierstein 25, 27, 31, 78 Antoine Rivoire ............. 31, 74 Archie Gray ................. 70, i4 Ards Rover ...............21, 31, is Arthur R. Goodwin ........ 31, 76 Aschenbrödel .............. 1 $1 ;, 31,7$ Augustine Guinoissea u ... 19, 24, $28,31,74$

Augustus Hartmann ... 18, 23, $25,31,74$ Austrian Copper ........ 25, 31, 72 Austrian Yellow ........ 25, 31, 72 Aviateur Bleriot ............. 32, 78 Avaca $\ldots 19,21,22,24,25,32,74$ Banksia alba ................ 32, 72

Banksia lutea ........... 21, 32, 72 Benedicte Sequin ............. 32, 74 Bennett's Seedling 21, 28, 32, 72

Bessie Brown $25,32,74$ Betty $18,26,32,74$ Blanc double de C'oubert 22, 32, 7 i Blanche Moreau 33,76 Blush Rambler ..... 24, 26, 33, 76 Bouquet d'Or ............. 21, 33, 78 Canarienvogel $18,33,77$ Candeur Lyonnaise ...... 25, 33, 73 Captain Georges Dessirier ... 70, 74 Captain Hayward ........... 33, 73 Carmine Pillar ... 21, 25, 33, 76 Caroline Testout 17, 19, 23, 25, 33, 74 Caroline Testout, Climbing 21,

$24,33,74$

Cecile Brunner ................ 34, 77

Celestial ....................... 34, 72

Charles E. Shea ..... 1i, 23, 34, 74 Charles K. Douglas ........... 34, 74 Charlotte Klemm ............. 34, 72 Château de Clos Vougeot 18, 19

$24,34,74$
Chatillon Rambler ..... $21,34,78$ Cheerful ...................... 34, 74 Cherry Page ........ 17, 26, 34, 74 China, Common ............ 34, 72 Chrissie Mackellar ..... 18, 34, 74 Christine $\quad \ldots \ldots \ldots . .18,26,35,76$ Christine Wright ....... 21, 35, 78 Clarice Goodacre ............. 35, 74 Clytemnestra ....................36, 73 Colonel Oswald Fitzgerald 18, 36, 74 Commandant Beaurepaire ... 35, 72 Commandant. Félix Faure 24, 35, 73 Common China ............. 34, 72 Common Moss 56,76 
Common Provence $25,28,77$

Common Sweetbriar 66,78 Comtesse du Cayla $18,20,35,72$ Conrad F. Meyer $21,22,23$, $24,36,77$

Constance $26,36,76$

Coral Cluster 70,77 Corallina 36,78

Coronation $23,25,36,73$

Coronation 36,78

Countess of Shaftesbury 25, 36, 74 Countess of Warwick 36,74 Covent Garden 36,74 Cramoisie Supérieure, Climbing 36,72

Crested: Moss 36,76

Crimson China 37

Crimson Rambler 37,76 Cupid $26,37,74$

C. V. Haworth 37,74

Cynthia Forde $19,37,74$ Danäe $19,22,26,37,73$ Dean Hole $23,25,37,74$ Débutante $20,37,78$

Diabolo $21,25,37,79$

Donald McDonald ..... 18, 37, 74 Dorothy Page-Roberts ... 17, 38, 74 Dorothy Perkins ... 20, 22, 24, $26,28,38,79$

Dowager Countes.5 of Roden 38, 74 Dr. Joseph Drew ... 24, 25, 38, 74 Dr. Van Fleet ............... 38, 79 Duchess of Wellington 24, 26, 38, 74 Duchess of Westminster ... 38, 74 Dundee Rambler ........ 21, 38, 72 Ecarlate .......... 18, 19, 26, 38, 74 Edgar M. Burnett ..... 25, 39, 74 Edel ........................ 23, 39, 74 Edith Cavell 39,74 E. Godfrey Brown 39, 74
Elegante 39,74 Elisa Robichon ........... '20, 39, 79 Ellen Poulsen 18, 20, 24, 26, 39, 77 Emily Gray ............... 21, 39, 76 Emma Wright .......... 18, 39, 74 Ethel ............................ 39, 79 Eugénie Lamesch ........ 24, 39, 77 Evangeline ..... 20, 24, 25, 39, 79 Excelsa ..... 20, 21, 24, 26, 39, 79 Fabvier .................... 18, 40, 72 Félicité-et-Perpétue ..... 21, 40, 77 Fellenberg .................... 22, 40, 72 Flame of Fire ........... 18, 40, 74 Flora McIvor .............. 27, 40, 78 Florence Forrester 23, 25, 40, 74 Florence H. Veitch 19, 21, 22,

$24,41,74$

Florence Pembertion ... 25, 41, 74 . Florinda Norman Thompson 70, 74 Fartune's Yellow ........ 24, 41, 76 Frances Gaunt ................ 41, 74 Francklin ...................... 70, 76 François Crousse ............. 41, 74 Francois Guillot ............. 41, 79 François Juranvilie 20, 24, 41, 79 Frau Karl Druschki 17, 19, 22, $23,25,41,73$ Gardenia ............ 22, 26, 41, 79 G. C. Waud ............ 18, 42, 74 Général Jacqueminot $19,24,42,73$ Gemeral McArthur 18, 19, 23, $24,26,42,74$ George Dickson ... 19, 23, 26, 42, 74 George Elgar ..................... 42, 77 Gerbe Rose $21,42,79$ Gladys Holland 42,74 Gloire de Chédane-Guinoisseau $26,42,73$ Gloire de Dijon $21,42,78$ Glowworm $25,70,76$ 
G. Nabonnand $17,43,78$

Joseph Lamy 47,79 Golden Emblem $18,19,43,76$ Julia Mannering $27,47,78$ Golden Ophelia … 18, 43,74 Golden Spray 43,74 Goldfinch 43,76 Gorgeous $18,19,23,26,43,74$ Gruss an Teplitz 19, 21, 22, 23, 43, 74 Gustar Grünerwald 17, 19, 23,

$24,43,74$

Gustave Régis $19,22,26,43,74$, Hadley $18,44,74$

Harrisonii 44,72

I. D. M. Barton 44,74

Hebe's Lip 44,77

Helen Chamberlain 44,74 Henrietta $18,26,44,74$

Hiawatha $20,24,25,26,44,79$ Hoosier Beauty ......... 18, 23, 44, 74 Horace Vernet ............. 26, 44, 73 F. P. Pinkerton ............... 44, 74 IIugh Dickson 19, 21, 22, 23, 24,

$26,45,73$

Hugonis $22,25,45,77$

H. $\nabla$. Machin $23,26,45,74$

Independence Day 45, 76

Irish Afterglow $\overline{45}, 74$ Irish Elegance ... 18, 22, 25, 26, 45, 74 Irish Fireflame ... 18, 25, 26, 45, 74 Irish Fireflame, Olimbing ... 45, 74 Isobel ............. 18, 19, 25, 26, 45, 76 Janet's Pride 45,78

J. B. Clark ... 19, 21, 22, 23, 26, 46, 75 Jean Guichard 46,79 Jeanie Deans $27,46,78$

Jersey Beauty $22,25,26,46,79$ Jessie $18,20,24,26,46,77$ Joanna Bridge 46,75 Jonkheer J. L. Mock ... 24, 26, 46, 75 Joseph Billard $20,25,46,79$ Joseph Hill $18,19,26,47,75$ $\mathrm{K}$. of $\mathrm{K} \ldots \ldots \ldots \ldots .18,19,26,47,75$ Kootenay ........................ 47, 75 Kosters Orleans ................ 70, 77 Lady Alice Stanley ... 18, 24, 47, 75 Lady Ashtown ...... 17, 19, 26, 47, 75 Lady Ashtown, (limbing 21, 24,

47,74

Lady Curzon ........ 22, 25, 27, 48, 72 Lady Gay ........ 20, 22, 24, 27, 48, 79 Lady Godiva ... 20, 22, 24, 27, 48, 79 Lady Greenall .................. 47, 75 Lady Fillingdon 18, 19, 23, 27, 48, '18 Lady Hillingdon, Climbing 21 $24,48,78$ Lady Maureen Stewart ..... 70, 75 Lady Penzance ...... 22, 25, 27, 48, 78 Lady Pirrie ........ 18, 19, 23, 48, 75 Lady Plymouth ........ 23, 26, 49, 78 Lady Roberts ...... 18, 19, 23, 49, 78 Lady Waterlow ... 19, 21, 22, 49, 75 La France ............... 19, 24, 49, 75 Lamia 49,75

La Tosca ... 17, 19, 22, 23, 26, 49, 75 Laurent Carle ... 18, 19, 20, 24, 49, 75 Laurette Messimy ........ 17, 49, 72 Lemon Pillar ................ 21, 49, 73 Léonie Lamesch ....... 20, 22, 49, 77 Léontine Gervais ..... 24, 27, 50, 79 Liberty ...................... 24, 50,75 Lieutenant Chauré 18, 23, 24, 50, 75 Lilian Moore .................... 50, 75 Little Meg .................. 17, 50, 77 Lord Penzance ............. 27, 50, 78 Los Angeles $18,27,50,75$

Louise Baldwin 50,75 Louise Catherine Breslau 18, 19 , $27,50,76$ 
Louise Crette $19,23,26,50,73$

May Marriott 54,76

lucida 51,77

lucida plena 51,77 Medea $26,54,78$

Lulu 70,75

Lyon Rose $19,26,51,76$

Mabel Drew $26,51,75$ macrantha $22,25,51,77$

Mme. Abel Chatenay 17, 19, 23, $24,27,51,75$

Mme. Abel Chatenay, Climbing

$$
21,51,74
$$

Mme. Alfred Carrière 21, 24, 51, 73

Mme. Antoine Mari ... 17, 27, 52, 78 Mme. C. Martel ................ 52, 75 Mme. Constant Soupert ... 26, 52, 78 Mme. d'Arblay 52,73

Mme. Edouard Herriot 18, 19, 23, $27,52,76$

Mme. Eugéne Resal ... 18, 20, 52, 72 Mme. Jean Dupuy ........ 19, 52, 78 Mme. Jules Bouché ........ 17, 52, 75 Mme. Jules Gouchault ........ 52, 77 Mme. Jules Gravereaux 22, 26,

Mme. Jules Grolez ............ 53, 75 Mme. Léon Pain ... 17, 23, 27, 53, 75 Mme. Ravary ... 18, 19, 23, 27, 53, 75 Mme. Segond-Weber ... 17, 19, 53, 75 Maiden's Blush ............... 53, 72 Maman Cochet ........ 19, 26, 53, 78 Maman Turbat .............. 17, 53, 77 Manifesto ........................ 70,75 Marcella ............................ 53, 75 Marchioness of Ormonde ..... 53, 75 Maréchal Niel ..... 21, 24, 26, 53, 76 Margaret ......................... 54, 75 Margaret Dickson Hamill 18, 19,

Marie Pavie 54,77 Marie van Houtte $18,19,54,78$ $27,54,78$ Mélanie Soupert 18, 19, 23, 26, 54, 75 Mélanie Soupert, Climbing 21,

$24,54,74$ Melody ....................... 23, 55. 75 Mermaid ... 19, 21, 22, 25, 27, 55, 73 Mildred Grant .............. 26, 55, 75 Minnehaha ........ 20, 24, 27, 55, 79 Miriam ............................. 55, 75 Miss Willmott ................. 55, 75 Modesty $19,26,55,75$ Molly Bligh 55,75 Molly Sharman-Crawford 17, 19, $23,26,55,78$

Moonlight $19,22,27,55,73$ moschata $22,56,77$ moschata alba $25,56,77$ moschata fl. pl 56,77 moschata himalayica ........ 56, 77 Moss, Common ................... 56, 76 Moss, Crested ................... 56, 76 Moss, Perpetual White ..... 56, 76 Moyesii ............ 22, 25, 27, 56, 77 Mrs. Aaron Ward ............. 56, 75 Mrs. A. E. Coxhead 19, 24, 26, 56, 75 Mrs. Alfred Tate ..... 17, 27, 57, 75 Mrs. Ambrose Ricardo ........ 57, 76 Mrs. Arthur Johnson ........ 70, 75 Mrs. Bertram J. Walker ..... 57, 75 Mrs, Bryce Allan ........ 24, 57, 75 Mrs, Campibell Hall ........... 57, 78 Mrs. Charles Lamplough ... 70, 75 Mrs. Curnock Sawday ........ 70, 75 Mrs. C. Russell ........... 26, 57, 75 Mrs. C. V. Haworth ........ 57, 75 Mrs. Dunbar Buller ........ 57, 75 Mrs. Dunlop Best .......... 57, 75 Mrs. E. G. Hill ..... 18, 27, 57, 75 
Mrs. E. Powell 18, 19, 23, 27, 58, 75 Mrs. Elisha J. Hicks ..... 58, 75 Mrs. Farmer .................. 58, 76 Mrs. Holey Hobibs 20, 23, 24, $26,58,78$ Mrs. Franklin Dennison 26, 58, 75 Mrs, F. W. Flight ............... 24. Mrs. G. Marriott ............. 58, 75 Mrs. George Norwood 24, 26, 58, 75 IIrs. George Shawyer 18, 20, $23,58,75$ Mrs. H. D. Greene ..... 27, 58, 75 Mrs. H. R. Darlington ...... 70, 75 Mrs. Henry Morse ........... 58, 75 Mrs. Herbert Stevens 17, 20, $23,27,59,78$ Mrs. J. H. Welch ..... 26, 59, 75 Mrs. John Laing 17, 20, 23, $24,26,59,73$

Mrs. Mackellar 59,75

Mrs. Mona Hunting 59,75

IIrs. O. G. Orpen 59,72

Mrs. R. D. MIeClure ... 26, 59, 75 Mrs. Redford 59,75

Mrs. Rosalie Wrinch ..... 59, 75 Mrs. Theodore Roosevelt 26, 59, 75 Mr's. Wakefield Christie-Miller $17,60,75$

Mrs. Wemyss Quin 18, 20, 27, 60, 76 Mrs. W. H. Cutbush 17, 20, 24 $27,28,60,77$

Mrs. W. H. Cutbush, Climbing 60,77

Mrs. W. J. Grant, Climbing 24, 74 Mrs. W. Lindsay ........... 70, 75 multiflora ........................... multiflora grandiflora ........ 25, 60 Nellie Parker ........... 17, 60, 75 Niphetos, Climbing ... 24, 61, 78 Noblesse 61,75 Nova Zembla.
Old Crimson China 61,72 Old Golk ....... 18, 20, 27, 61, 75 Oplelia ... 17, 20, 23, 24, 27, 61, 75 Orleans Rose 18, 20, 24, 27, 61, 77 Orleans Rose, Climbing ... 61, 75 Papa Gontier, Climbing 21, 61, 74 Paul Lédé, Climbing 21, 24, 61, 74 Pax ............ 19, 22, 27, 62, 73 Perle d'Or ........... 18, 20, 62, 77 Persian Yellow ................ 62, 72 Petit Constant ................ 62, 77 Plharisäer ... 17, 20, 23, 24, 62, 75 pimpinellifolia $\ldots . . . . . . . .662,77$ Pissardii ...................... 62, 77 polyantha grandiflora ..... 62, 77 polyantha simplex .......... 62, 77 pomifera $\ldots . . . \ldots \ldots \ldots \ldots \ldots \ldots . . . \ldots 62,78$ President Bouché ............. 63, 76 President Wilison ............. 63, 75 Prima Donna ..................6 63, 75 Prince Charming ............ 63, 76 Prince de Bulgarie 17, 20, 23, 63, 76 Princess Mary ........... 25, 63, 76 Provence, Common ........ 24, 63 Provence, White ................. 63 Purity $20,22,76$ Queen Mab .................... 63, 72 Queen of Musk ............... 64, 73 $R$. arvensis .................... 64, 72 Raymond ........................ 64, 76 Red Cross .................... 64, 76 Red Letter Day ........ 27, 64, 76 Refulgence ......................64, 64, 78 Reine OLga de IVurtemberg 64, 76 René André ............. 20, 64, 79 Rêve d' $\mathrm{O}_{\mathrm{r}} \ldots \ldots \ldots \ldots \ldots \ldots . . . .21,64,76$ Richmond ,.. 18, 23, 24, 27, 64, 76 Richmond, Climbing .......... 24. Rödhätte .................. 18, 65, 77 Rasa Mundi ................ 27, 65, 73 Rubella 65,78 
rubrifolia 65,78

rugosa alba $25,65,77$ rugosa subra $20,24,65,79$ Sanders White Sarah Bernhardt ... 21, 22, 65, 76
Scarlet Climber (Paul's) 19, 21, $24,27,65,76$

Seagull 65,76 sericea 66,78 sericea, pteracantha 65,78 Severine 66,76

Shower of Gold $21,66,79$ sinica Anemone $21,25,66,78$ Source d'Or 66,79

Stanwell Perpetual 22, 24, 66, 77 Sunburst $23,66,76$ Sunburst, Climbing $21,66,74$ Sweet Briar, Common ...... 66, 78 Tausendschön $24,66,76$ Tea Rambler 67, 76

T. F. Crozier 67,76

The Garland 67,73

The Queen Alexandra Rose 24, $27,67,76$

Thoreshyana 67

Toreador 67, 76

Trier
Triomphe d'Orleanaise 67, 77 Ulrich Brunner 23, 24, 26, 67, 73 Una .................... 25, 27, 67, 73

Victor Hugo .................. 67, 73

$\nabla$ iscountess Folkestone 17, 20, 68,76

Victory ........................ 70, 76

W. C. Clark ................... 68, 76

W. C. Gaunt ................. 68, 76

White Dorothy ,.... 21, 24, 68, 83 White Maman Cochet 20, 26, 68,78

White Provence .............68, 68, 77 White Rambler (Pemberton's)

68,73 wichuraiana ............ 25, 68, 79 William Allen Richardson 20, $21,22,24,68,76$ William Shean ..... 23, 26, 69, 76 W. R. Smith ........ 20, 26, 69, 78 xanthina $\quad \ldots \ldots \ldots \ldots \ldots \ldots .25,69,78$ York and Lancaster ........ 69, 72 Yvonne Rabier ..... 17, 22, 69, 77 Yvonne Vacherot ............. 69, 76 Zéphirine Drouhin 21, 22, 23, $24,69,73$ 


\section{The National Rose Society's \\ OFFICIAL LIST OF ROSES.}

\section{INTRODUCTORY REMARKS.}

A FEW explanations may be advisable as to the terms employed in the descriptions of the different varieties included in this List. In the first place, after the name of each Rose is given (1) the class to which it belongs ; (2) the raiser's or introducer's name and the date of introduction; (3) the colour of the flower; (4) the character of the growth, whether vigorous, moderate, or otherwise: (5) the purposes for which it is best suited; (6) general remarks and pruning references.

\section{1.-The Class to which it belongs}

The following abbreviations are used: Ayr., Ayr. shire. B.,Bourbon. C., China. D., Damask. H.B., Hybrid Bourbon. H. Brac., Hybrid Bracteata. H. Briar, Hybrid Briar. H.N., Hybrid Noisette. H.P., Hybrid Perpetual. H.T., Hybrid Tea. mult. ramb., multiflora ramblers (climbing polyantha). Pernet., Pernetiana or Old Austrian Hybrid. N., Noisette. poly. pom., polyantha pompon. Prov., Prowence. rug., rugosa. S. Briar, Sreet Briar and Hybrids. 'T., Tea. wich. ramb., wichuraiana Rambler. wich. pom., richuraiana pompon.

Single=flowered Roses.

By "single-flowered" is meant a Rose having only one row of peta!s, by "semi-single" two rows of petals, and by "semi-double" n110re than two rows of petals but not altogether full. 


\section{4.-The Character of the Growth :}

The terms adopted range from weak to very rampant growth and are arranged as follows : Weak, moderate, medium, moderately vigorous, robust, vigorous, and very vigorous. Where the growth is sturdy but short it is described as " robust." In the case of climbing Roses those suitable for a dwarf pillar are diescribed as " semi-climbers" ; those suitable for a tall pillar or pergola as " very vigorous climbers."

It must be borne in mind that these terms are used in relation to the class to which the Rose belongs. For instance, a Tea Rose may rightly be described as vigorous, whereas the same habit in a Hybrid Perpetual or Hybrid Tea would be described as moderately vigorous.

\section{5.-The purpose for which the Rose is best suited:}

Arch.-That it is suitable for growing over an arch.

Bedding.-That it is a good variety for massing together in a single bed.

Bush.-That when planted by itself and allowed to develop it makes a bold and shapely bush.

Creeping. - That is is well adapted for trailing over a bank. 
Decorative. - That it is specially useful for Vase and other cut flower purposes.

Exhibition.-By this is meant a variety which is of too poor growth, or is otherwise unsuited, for general garden cultivation, although in the hands of an experienced exhibitor it may be made to produce fine exhibition blooms.

Garden.-That the variety is recommended for general garden cultivation.

Glass.-That it is well suited for growing under glass, either in pots or planted out.

Hedge.- That it is well suited for making a hedge.

Pergola. - That it is well adapted for training over a pergola.

Pillar.-That it is excellent for training up posts or pillars of varying height.

Screen.-That it is suitable for training up trellis work or other framework used as a screen.

Standard.-That it makes a good standard.

Town.-That it is one of the best Roses for growing in a town or suburban garden.

Wall.-That it is suitable for training up the face of a wall or the side of a house.

Weeping Standard.-That it makes an excellent weeping standard. 


\section{6.-Summer=flowering Roses :}

By summer-flowering is meant those Roses which flower only once in the year, even though flowering late.

\section{7.-Climbing Sports from Dwarf Roses :}

These climbing sports are not always dependable, as one plant of the same variety will make strong climbing shoots, while another will revert in growth to the variety from whicb it sported.

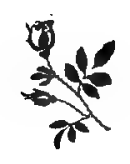




\section{SELECTIONS OF ROSES}

For various purposes mentioned in List.

\section{Selection 1.}

\section{BEDDING ROSES.}

\section{ARRANGED APPROXIMATELY BY COLOUR.}

NoTE.-It is recommended that beds be made of one variety only.

\section{White and Cream.}

Frau Karl Druschki (H.P.)

Katharine Zeimet (poly. pom.)

Little Meg (poly. pom.)

Mme. Jules Bouché (H.T.)
Molly Sharman-Crawford (T.)

Mrs. Herbert Stevens (T.)

Yronne Rabier (poly. pom.)

\section{Blush and Flesh.}

G. Nabonnand (T.)

La Tosca (H.T.)

Mme. Antoine Mari (T.)

Nellie Parker (H.T.)
Ophelia (H.T.)

Pharisäer (H.T.)

Prince de Bulgarie (H.T.)

Viscountess Folkestone (H.T.) -

\section{Pink and Salmon Pink.}

Aschenbrödel (poly. pom.)

Caroline Testout (H.T.)

Charles E. Shea (H.T.)

Cherry Page (H.T.) (semi-double) Dorothy Page-Roberts (H.T.)

Gustav Grünerwald (H.T.)

Lady Ashtown (H.T.)

Laurette Messimy (C.)
Maman Turbat (poly. pom.)

Mme. Abel Chatenay (H.T.) -

, Segond-Weber (H.T.)

, Léon Pain (H.T.)

Mrs. Alfred Tate (H.T.)

, W. Christie-Miller (H.T.)

"W. H. Cutbush (poly. pom.) 


\section{Rose.}

Donald McDonald (H.T.)

Ellen Poulsen (poly. pom.)

-Lady Alice Stanley (H.T.)

Mme. E. Resal (U.)
Mrs. E. G. Hill (H.T.)

George Shawyer (H.T.) -

Orleans Rose (poly. pom.)

\section{Light Crimson.}

Augustus Hartmann (H.T.)

Ecarlate (H.T.)

"General MeArthur (H.T.)

G. C. Waud (H.T.)

Jessie (poly. pom.)
Laurent Carle (H.T.)

Mrs. I. Powell (H.T.)

Richmond (H.T.)

Rödhätte (poly. pom.)

\section{Dark Crimson.}

Admiral Ward (H.T.)

Château de Clos Vougeot (H.T.)

Colonel Oswald Fitzgerald (H.T.)

Fabvier (C.)

Hadley (H.T.)
Hoosier Beauty (H.T.)

K. of K. (H.T.) (semi-single)

Lieut. Chauré (H.T.)

Red Letter Day (II.T.) (semi-do

\section{Yellow.}

Canarienvogel (poly. pom.)

Christine (Pernetiana)

Golden Emblem (Pernetiana)

Golden Ophelia (H.T.) "

Lady Hillingdon (T.)
Mme. Ravary (H.T.)

Margaret Dickson Hamill (H.T. Mrs. Wemyss Quin (Pernetian: Mélanie Soupert (H.T.)

Perle d'Or (poly. pom.)

\section{Copper Shaded.}

Betty (H.T.)

Chrissie Mackellar (H.T.) (semidouble)

Comtesse du Cayla (C.)

Emma Wright (II.T.)

Flame of Fire (H.T.)

Gorgeous (H.T.)

Henrietta (H.T.) (semi-double)

Irish Elegance (H.T.) (single)

, Firetlame (H.T.) (single)
Isobel (Pernetiana) (single)

Joseph IIill (H.T.)

Lady Pirrie (H.T.)

Roberts (T.)

Los Angeles (H.T.)

Louise Catherine Breslan (Pernetiana)

Mme. E. Herriot (Pernetiana)

Old Gold (H.T.) 


\section{Tall Roses for Beds.}

Alister Stella Gray (N.)

Caroline Testout (H.T.)

Danäe (Hybrid Musk)

Florence H. Veitch (H.T.)

,Frau Karl Druschki (H.P.)

Gruss an Teplitz (H.T.)

Gustave Régis (II.T.)

Hugh Dickson (H.P.)

J. B. Clark (H.T.)
La Tosca (H.T.)

Lady Waterlow (H.T.)

Mermaid (H. Brac.) (single)

Moonlight (Hybrid Musk)

Pax (H, Musk)

Scarlet Climber (Paul's) (H. wich.)

Trier (Hybrid Musk)

Zéphirine Drouhin (H.B.)

\section{Selection 2.}

\section{ROSES FOR STANDARDS.}

A. Hill Gray (T.)

Aruna Olivier (T.)

Augusline Guinoisseau (H.T.)

Avoca (H.T.)

Caroline Testout (H.T.)

Château de Clos Vougeot (H.T.)

Cynthia Forde (H.T.)

Ecarlate (II.T.)

Florence H. Veitch (H.T.)

Frau Käl Druschki (H.P.)

Général Jacqueminot (H.P.)

General McArthur (H.T.)

George Dickson (H.T.)

Golden Emblem (Pernetiana)

Gorgeous (H.T.)

Gruss an 'l'eplitz (H.T.)

Gustav Grünerwald (H.T.)

Gustave Régis (HI.T.)

Hugh Dickson (II.P.)

Isobel (Pernetiana) (single)

J. B. Clark (H.T.)

Joseph Hill (H.T.)

K. of K. (H.T.) (semi-single)

Lady Ashtown (H.T.)
Lady Hillinglon (T.)

, Pirrie (H.'I'.)

, Roberts (T.)

, Waterlow (H.T.)

La France (H.T.)

La Tosea (H.T.)

Laurent Carle (H.T.)

Louise Catherine Breslau

(Pernetiana)

Louise Cretté (H.P.)

Lyon Rose (Pernetiana)

Maman Cochet (T.)

Marie van Houtte (T.)

Margaret Dickson Hamill (H.'T.)

Mélanie Soupert (H.T.)

Mme. Abel Chatenay (H.T.)

, E. Herriot (Pernetiana)

, Jean Dupuy (T.)

, Ravary (I.T.)

, Segond-IVeber (H.T.)

Modesty (H.T.)

Molly Sharman-Crawford (T.)

Mrs. A. E. Coxhead (H.T.)

, E. Powell (H.T.) 


\section{ROSES FOR STANDARDS-contiuued.}

Mrs. Foley Hobbs (T.)

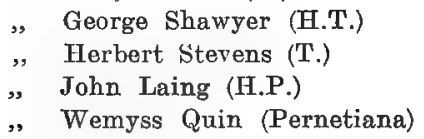

Old Gold (H.T.)

Ophelia (H.T.)
Pharisaer (H.T.)

Prince de Bulgarie (H.'T.)

Vissountess Folkestone (H.T.)

White Maman Cochet (T.)

W. R. Smith (T.)

William Allen Richardson (N.)

\section{Polyantha Pompon.}

(This class may also be grown as Dwarf Standards.)

Ellen Poulsen

Jessie

Katharine Zeimet

Léonie Lamesch
Mrs. W. H. Cutbush

Orleans Rose

Perle d'Or

\section{China.}

Comtesse du Cayla

Mme. E. Resal

Laurette Messimy

\section{Selection 3.}

\section{ROSES FOR WEEPING STANDARDS.}

Albéric Barbier (wich.)

Débutante (wich.)

Dorothy Perkins (wich.)

Elisa Robichon (wich.)

Evangeline (wich.)

Excelsa (wich.)

François Juranville (wich.)

Hiawatha (wich.)
Joseph Billard (wich.) (single)

Lady Gay (wich.)

" Godiva (wich.)

Minnehaha (wich.)

Purity (H. wich.)

Réné André (wich.)

Sanders White (wich.)

And most of the other wichuraiana Ramblers. 


\section{Selection 4.}

\section{ROSES FOR WALLS.}

\section{For Walls facing North.}

Albéric Barbier (wich.)

Bennett's Seedling (Ayr.)

Bouquet d'Or (Dijon T.)

Conrad F. Meyer (rug.)

Dundee Rambler (Ayr.)
Félicité-et-Perpétue

(Sempervirens)

Gloire de Dijon (T.)

Mermaid (H. Brac.)

\section{For Walls facing East.}

Ards Rover (H.P.)

Caroline Testout (climbing) (H.T.)

Florence H. Veitch (H.T.)

Gloire de Dijon (T.)
Gruss an Teplitz (H.T.)

Lady Waterlow (H.T.)

Mme. Alfred Carrière (H.N.)

Mermaid (H. Brac.)

\section{For Walls facing South or East.}

Alister Stella Gray (N.)

Banksia Yellow

Lady Ashtown (climbing) (H.T.)

Lady Hillingdon (climbing) (T.)

Maréchal Niel (N.)

Mélanie Soupert (climbing) (H.T.)

Mermaid (H. Brac.)
Papa Gontier (climbing) (H.T.)

Paul Lédé (climbing) (H.T.)

Rêve d'Or (N.)

sinica Anemone (H. of Species)

Sunburst (climbing) (H.T.)

W. A. Richardson (N.)

Zéplirine Drouhin (H.B.)

\section{Selection 5.}

\section{ROSES FOR PILLARS.}

(The varieties marked with an * are perpetual flowering.)

American Pillar (wich.)

*Ards Rover (H.P.)

*A voca (H.T.)

Carmine Pillar (mult. ramb.)

Chatillon Rambler (wich.)

*Christine Wright (wich.)

Conrad F. Meyer (rug.)

Diabolo (wich. ramb.)

Emily Gray (H. wich.)

Excelsa (wich.)

*Florence H. Veitch (H.T.)
Gerbe Rose (wich.)

*Gruss an Teplitz (H.T.)

*Hugh Dickson (H.P.)

*J. B. Clark (H.T.)

*Lady Waterlow (H.T.)

Lemon Pillar (H.N.)

*Mme Abel Chatenay (climbing)

*Sarah Bernhardt (H.T.)

*Scarlet Climher (Paul's) (H. wich.)

Shower of Gold (wich.)

*Zéphirine Drouhin (H.B.) 


\section{Selection 6.}

\section{ROSES FOR COVERING BANKS.}

Albéric Barbier (wich.)

Dorothy Perkins (wich.)

Gardenia (wich.)
Jersey Beauty (wich.) (single)

Lady Gay (wich.)

Lady Godiva (wich.)

And most of the wichuraian as including the Species.

\section{Selection 7.}

\section{ROSES FOR PEGGING DOWN.}

Avoca (H.T.)

Florence U. Veitch (IT.T.)

Frau Karl Druschiki (H.P.)

Hugh Dickson (H.P.)
J. B. Clark (H.T.)

Lady Waterlow (H.T.)

Mme. Jules Graverean ('T.)

W. A. Richardson (N.)

\section{Selection 8.}

\section{ROSES FOR FORMING A HEDGE.}

altaica (Species) (single)

Danäe (Hybrid Musk)

Fellenberg (C.)

Gruss an Teplitz (II.T.)

Lady Curzon (Damask) (single)

Moschata (Species) (single)

Moonlight (Hybrid Musk)
Penzance Briars

Purity (H. wich.)

Rugrosa Roses and their Hybrids

Scotch Ruses

Stanwell Perpetual (Perpetual Scotch)

Zéphirine Drouhin (H.B.)

\section{Selection 9. \\ ROSES FOR GROWING AS BUSHES}

altaica (Species) (single)

Blanc double de Coubert (rug.) (semi-double)

Conrad F. Meyer (rug.)

Danäe (Hybrid Musk)

Fellenberg (C.)

Florence II. Veitch (H.T.)

Gruss an Teplitz (П.T.)

Gustave Régis (H.T.)

Hugonis (Species) (single)

Trish Elegance (H.T.) (single)

La Tosca (H.T.)

Lady Penzance (S. Briar) (single)
Lady Waterlow. (H.T.)

Léonie Lamesch (poly. pom.)

macrantha (Hybrid of Species)

(single)

Mermaid (H. Brac.) (single)

Moonlight (Hybrid Musk)

Moyesii (Species) (single)

Nova Zembla (rug.)

Pax (H. Musk)

Sarah Bernhardt (H.T.)

Trier (Hybrid Musk)

Yvonne Rabier (poly. pom.) 


\section{Selection 10.}

\section{ROSES FOR GROWING IN TOWNS.}

Caroline Testout (II.T.)

Conrad F. Meyer (rug.)

Frau Karl Druschli (E.P.)

General MeArthur (H.T.)

Gruss an Teplitz (H.T.)

Gustav Grünerwald (H.T.)

Hurh Dickson (H.P.)

J. B. Clark (II.T.)

La Tosca (H.T.)
Mme Edouard Herriot (Pernetianap

n Léon Pain (H.'Г.)

" Ravary (H.T.)

Mrs. E Powell (IL.T.)

Mrs. John Laing (HI.P.)

Prince de Bulgarie (H.T.)

Ulrich Binner (IL.P.)

Zéphirine Drouhin (II.B.)

\section{Selection 11.}

\section{ROSES FOR GROWING UNDER GLASS.}

\section{Cut Flowers.}

Charles E. Shea (H.T.)

Frau Karl Druschki (H.P.)

Hoasier Beauty (H.T.)

Lady Hillingrdon (T.)

, Pirrie (II.T.)

,. Roberts (T.)

Liberty (II.T.)

Mme. Abel Chatenay (H.T.)

E. Herriot (Pernetiana)
Melody (II.'I.)

Molly Sharman-Crawford ('T.)

Mrs. George Shawyer (H.'I.)

, Herbert Stevens (T.)

,J John I.aing (II.P.)

Ophelia (H.T.)

Richmond (II.T.)

Sunburst (H.T.)

\section{Exhibițion Blooms.}

Alexander Hill Gray (T.)

Augustus Hartmann (H.T.)

Caroline Testout (II.T.)

Coronation (H.P.)

Dean Ilole (H.T.)

Edel (HI.T.)

Florence Forrester (H.T.)

Frau F̆arl Druschki (II.P.)

George Dickson (H.T.)

Gargeous (H.T.)
H. V. Machin (H.T.)

Larly Plymouth (T.)

Lieut. Chauré (II.T.)

Louise Cretté (H.P.)

Mélanie Soupert (H.T.)

Molly Sharman-Crawford (T.)

Mrs. Fuley Hobbs

, John Laing (H.P.)

Pharisäer (II.T.)

William Shean (H.T.) 


\section{Specimen Plants for Indoor Decoration.}

American Pillar (wich.) (single)

Blush Rambler (muit. ramb.)

Durothy Perkins (wich.)

Ellen Poulsen (poly. pom.)

Excelsa (wich.)

Hiawatha (wich.) (single)

Jessie (poly. pom.)

Lady Godiva (wich.)
Lady Gay (wich.)

Minnehaha (wich.)

Mrs. F. W. Flight (mult. ramb.)

W. II. Cutbush (poly. pom.)

Orleans Rose (poly. pom.)

Sanders White (wich.)

Scarlet Climber (Paul's) (H. wich.)

Tausendschön (mult. ramb.)

\section{Roses for Greehouse Climbers.}

Caroline Testout (climbing) (H.T.) Fortune's Yollow (N.)

Lady Ashtown (climbing) (H.T.)

Lady Hillingdon (climbing) (H.T.)

Maréchal Niel (N.)

Mélanie Soupert (climbing) (H.T.'
Mrs. W. J. Grant (climbing) (H.T.)

Niphetos (climbing) (T.)

Paul Lédé (climbing) (H.T.)

Richmond (climbing) (H.T.)

William Allen Richardson (N.)

\section{Selection 12.}

\section{VERY FRAGRANT ROSES.}

Augustine Guinoisseau (H.T.)

Avoca (H.T.)

Château de Clos Vougeot (H.T.)

Commandant Félix Faure (H.P.)

Conrad F. Meyer (rug.)

Dr. Joseph Drew (H.T.)

Duchess of Wellington (H.T.)

Eugénie Lamesch (poly. pom.)

Evangeline (wich.)

Florence H. Veitch (H.T.)

François Juranville (wich.)

Général Jacqueminot (H.P.)

McArthur (H.T.)

George Dickson (H.T.)

Gustav Grünerwald (H.T.)

Hugh Dickson (H.P.)

Jonkheer J. L. Mock (H.T.)

Lady Alice Stanley (H.T.)

La France (H.T.)

Laurent Carle (H.T.)
Léontine Gervais (wich.)

Liberty (H.T.)

Lieut. Chauré (H.T.)

Maréchal Niel (N.)

Mme. Abel Chatenay (H.T.)

Alfred Carrière (H.N.)

Mrs. A. E. Coxhead (H.T.)

, Bryce Allan (H.T.)

, Foley Hobbs (T.)

, George Norwood (H.T.)

, John Laing (H.P.)

Ophelia (H.T.)

Pharisäer (H.T.)

Provence Common (Prov.)

Richmond (H.T.)

Stanwell Perpetual (Perpetual Scotch)

The Queen Alexandra Rose (H.T.)

Ulrich Brunner (H.P.)

Zéphirine Drouhin (H.B.) 


\section{Selection 13.}

\section{SINGLE=FLOWERED ROSES.}

(The varieties marked with an * are perpetual flowering.)

altaica (Species)

Andersoni (Hybrid of Species)

Anne of Geierstein (S. Briar)

Austrian Copper (Austrian Briar)

Yellow (Austrian (Briar)

Glow Worm (Pernetiana)

Hugonis (Species)

*Irish Elegance (H.T.)

*Irish Fireflame (H.'I.)
Isobel (Pernetiana)

Lady Curzon (Damask) , Penzance (S. Briar)

Lord Penzance (S. Briar)

Moyesii (Species)

*Princess Mary (H.T.)

rugosa alba (rug.)

xanthina (Species)

\section{Single=flowered Climbing Roses.}

American Pillar (wich.)

Carmine Pillar (mult. ramb.)

Diabolo (wich.) (semi-single)

Evangeline (wich.)

Hiawatha (wich.)

Jersey Beauty (wich.)

Joseph Billard (wich.) macrantha (Hybrid of Species) Mermaid (II. Brac.) moschata alba (Hybrid of Species) multiflora grandiflora (H. of Sp.) sinica Anemone (H. of Species). Una (H. Briar) (semi-single) wichuraiana

\section{Selection 14.}

\section{ROSES SUITABLE FOR EXHIBITION.}

(The varieties marked with an $*$ are best as maidens.)

Alexander Hill Gray (T.)

Argustus Hartmann (II.T.)

Avoca (H.T.)

Bessie Brown (H.T.)

*Candeur Lyonnaise (H.P.)

Caroline Testout (H.T.)

*Coronation (H.P.)
Countess of Shaftesbury (H.T.)

Dean Hole (H.T.)

Dr. Joseph Drew (H.T.)

Edgar M. Burnett (H.T.)

"Florence Forrester (H.T.)

Pemberton (H.T.)

Frau Karl Druschki (H.P.) 


\section{ROSES SUITABLE FOR EXHIBITION-cont inued.}

Gerge Dickson (H.T.)

Gloire de Chédane-Guinoisseau (H.P.)

Gorgeous (H.T.)

*Horace Vernet (H.P.)

IIugh Dickson (H.P.)

*H. V. Machin (H.T.)

J. B. Clark (H.T.)

Jonkheer J. L. Mock (H.T.)

Joseph Hill (H.T.)

Lady Ashtown (H.T.)

Plymouth (T.)

Louiso Cretté (II.P.)

*Lyon Rose (Pernetiana)

Mabel Drew (H.T.)

Maman Cochet (T.)

Biaréchal Niel (N.)

-Medea (T.)

Melanie Soupert (H.T.)
* Mildred Grant (H.T.)

Mme. Constant Soupert (T.)

Jules Gravereaux (T.)

Modesty (H.T.)

Molly Sharman-Crawford ('L.)

Mrs. A. E. Coxhead (H.'T.)

, Charles Russell (H.T.)

, Foley Hobbs (T.)

, Franlilin Dennison (H.T.)

, John Laing (H.P.)

" George Norwood (H.T.)

, J. H. Welch (H.T.)

, R. D. MeClure (H.T.)

T. Roosevelt (H.T.)

Ulrich Brunner (H.P.)

White Maman Cochet (T.)

*Tilliam Shean (H.T.)

W. R. Smith (T.)

\section{Selection 15.}

\section{ROSES SUITABLE FOR EXHIBITION IN DECORATIVE CLASSES.}

Albéric Barbier (wich.)

American Pillar (wich.)

Betty (II.T.)

Blush Rambler (mult. ramb.)

Cherry Page (II.T.) (semi-double)

Christine (Pernetiana)

Constance (Pernetiana)

Cupid (II.T.) (single)

Danäe (H. Musk.)

Dorothy Perkins (wich.)

Duehess of Wellington (H.T.)

Ecarlate (H.T.)

Ellen Poulsen (poly. pom.)

Excelsa (wich.)
Gardenia (wich.)

General McArthur (H.T.)

Gustave Réris (П.T.)

Itenrietta (H.T.) (semi-double)

Hiawatha (wich.) (single)

Irish Elegance (H.T.) (single)

Fireflame (H.T.) (sıngle)

Isobel (Pernetiana) (single)

Jersey Reauty (wich.)

Jessie (poly. pom.)

Katharine Zeimet (poly. pom.)

K. of K. (T.T.) (semi single)

La Tosca (H.T.) 


\section{ROSES SUITABLE FOR EXHIBITION IN DECORATIVE} CLASSES-continued.

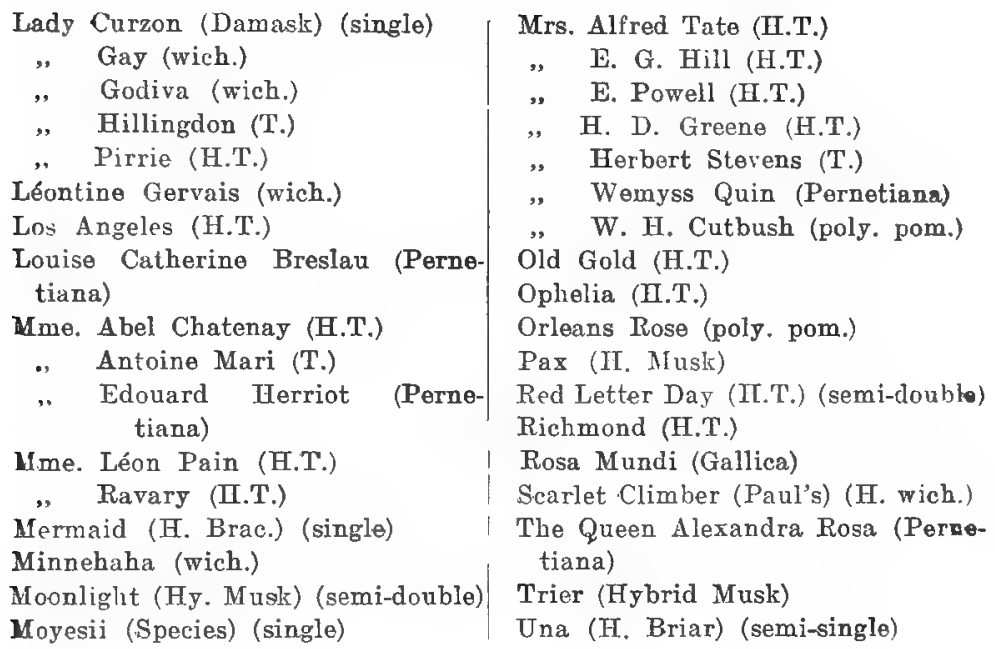
tiana)

Mme. Abel Chatenay (H.T.)

., Antoine Mari (T.)

, Edouard Herriot (Pernetiana)

Ime. Léon Pain (H.T.) Ravary (H.T.)

Mermaid (H. Brac.) (single)

Minnehaha (wich.)

Moonlight (Hy. Musk) (semi-double)

Moyesii (Species) (single)

Mrs. Alfred Tate (II.T.)

, E. G. Hill (H.T.)

, E. Powell (H.T.)

, H. D. Greene (H.T.)

, Herbert Stevens (T.)

"Wemyss Quin (Pernetiana)

, W. H. Cutbush (poly. pom.)

Old Gold (H.T.)

Ophelia (II.T.)

Orleans Rose (poly. pom.)

Pax (II. Musk)

Red Letter Day (IT.T.) (semi-double) Richmond (H.T.)

Rosa Mundi (Gallica)

Scarlet Climber (Paul's) (H. wich.)

The Queen Alexandra Rosa (Percetiana)

Trier (Hybrid Musk)

Una (H. Briar) (semi-single)

\section{Selection 16.}

\section{PENZANCE BRIARS.}

Amy Robsart

Anne of Geierstein

Flora McIvor

Jeanie Deans
Julia Mannering

Lady Penzance

Lord Penzance

Meg Merrilies 


\title{
ROSES TOO MUCH ALIKE
}

\section{For Exhibition Purposes.}

\begin{abstract}
Alfred Colomb (H.P.) .. $\ldots\left\{\begin{array}{l}\text { Marshall P. Wilder. } \\ \text { Wilhelm Koelle. }\end{array}\right.$
\end{abstract}

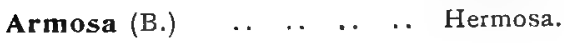

Augustine Guinoisseau (H.T.) White La France.

Bennett's Seedling (Ayr.) .. Thoresbyana.

Charles Lefèbvre (H.P.) . . $\left\{\begin{array}{l}\text { Marguerite Brassac. } \\ \text { Paul Janain. }\end{array}\right.$

Common China (C.) .. .. Old Monthly.

Common Provence (Prov.) .. The Old Cabbage Rose.

Dorothy Perkins (wich.) .. Lady Gay.

Duke of Wellington (H.P.).. Rosiériste Jacobs.

Fortune's Vellow (N.) .. Beauty of Glazenwood.

Inermis morletii (Boursault).. Mme. Sancy de Parabère.

Lady Faire (H.T.) .. .. Joseph Lowe.

Lady Godiva (wich.) . . . $\left\{\begin{array}{l}\text { Dorothy Dennison. } \\ \text { Christian Curle. }\end{array}\right.$

lucida plena (Species).. . . Rose Button.

Madame Wagram (H.T.) .. Comtesse de Turenne.

Maman,Levavasseur (poly.pom.) Baby Dorothy.

Marie Baumann (H.P.) ․ Madame A. Lavallée.

Mrs. W. H. Cutbush (poly.pom.) Mrs. Taft.

Mrs. W. J. Grant (H.T.) .. Belle Siebrecht.

pimpinellifolia (Species) .. The Burnet Rose.

Prince Camille de Rohan(H.T.) La Rosière.

setigera (Species) _. . . The Prairie Rose.

Souvenir de S. A. Prince (T.) The Queen.

Splendens (Ayr.) _. .. Myrrh Scented. 


\section{A DESCRIPTIVE LIST OF ROSES.}

N.B.-The Roses of recent introduction will be found at page 70.

Instructions for pruning are indicated by numbers

thus: Prune 4 or 5 means prune according to Instructions 4 or 5 .

For explanations of the terms used in this List see Introductory Remarks-Pages 13-16.

Admiral Ward (H.T.), Pernet-Ducher, 1915.Crimson red. Vigorous. Bedding. Prune 4.

Ennchen Müller (poly. pom.), J. C. Schmidt, 1907.-Bright pink. Medium. Garden, beđding. Petals reflexed. Large showy clusters. Subject to mildew. Fragrant. Prune 34.

Aglaia (mult. ramb.), Lambert, 1896.-Pale yellow. Very vigorous climber. Arch, pergola. Only flowers freely on established plants. Summer-flowering. Prune 27 or 39.

Aimée Vibert, Climbing (N.), Curtis, 1841.White. Vigorous climber. Pillar, pergola, wall. Almost evergreen. Late flowering, good in autumis. Prune 10, 32 (b) or 39.

Albéric Barbier (wich. ramb.), Barbier, 1900.Yellow buds, changing to creamy white. Vigorous climber. Pillar, arch, pergola, creeping, weeping standard, screen, N. wall. Early summer-flowering. Prune 37 or 39 .

Alexander Emslie (H.T.), A. Dickson \& Sons, 1918.-Velvety crimson. Moderately vigorous. Garden. Fragrant. Prune 4. 
Alexander Hill Gray ('T.), A. Dickson \& Sons, 1911.-Deep lemon yellow. Vigorous. Exhibition, garden, standard, glass. A good Tea. Fragrant. Prune 4,5 or 42 .

Alister Stella Gray (N.), (A. Hill Gray), Paul and Son, 1894.-Pale yellow, deeper centre. Vigorous climber. Standard, pillar, arch, pergola, S. or W. wall. Perpetual flowering in clusters. Fragrant. Small flower. Prune 32 (b) or 39.

alpina (Species), 1753.-Deep rose. Vigorous. Bush. Thornless, very early flowering. Singleflowered. Summer-flowering. Prune 28.

alpina pyrenaica (Species)._Bright rose. Moderate. Garden. A dwarf variety of " alpina." Very early. Single-flowered. Summer-flowering. Prune 28.

altaica (Species).-Pale lemon-white. Vigorous. Bush, hedge. Very early. Single flowered. Prune 28.

American Pillar (wich. ramb.), Conard \& Jones Company, 1909.-Bright rose, flesh centre. Vigorous climber. Pillar, arch, pergola. Single-flowered. Bold, glossy foliage. A fine variety. Summer-flowering. Prune 27 or 39.

Amy Robsart (S. Briar) (Lord Penzance), Keynes, Williams \& Co., 1894.-Deep rose. Very vigorous. Bush, hedge. Summer-flowering. Prune 22.

Andersoni (Hybrid of Species).--Rose pink. Vigorous. Bush. Single-flowered. Summer-flowering. Prune 29.

Anna Olivier (T.), Ducher, 1872.-Pale rosy flesh and buff. Vigorous. Exhibition, garden, standard. Fragrant. Prune 4 or 5. 
Anne of Geierstein (S. Briar) (Lord Penzance), Keynes, Williams and Co., 1894.-Deep crimson-rose. Very vigorous. Bush, hedge. Single-flowered. Summer-flowering. Prune 22 (b).

Antoine Rivoire (H.T.), Pernet-Ducher, 1896.Pale cream, with deeper centre. Moderately vigorous. Garden, standard, bedding. Fine early in the season. Good in autumn. Prune 4.

Ards Rover (H.P.), A. Dickson \& Sons, 1894.Dark crimson. Vigorous climber. Pillar, E. wall, trellis. A good crimson climber. Prune 10 or 39.

Arthur R. Goodwin (Pernet), Pernet-Ducher, 1910.-Coppery orange, passing to flesh. Moderately vigorous. Garden. Pretty in the bud. Prune 20.

Aschenbrödel (poly. pom.), Lambert, 1902.-Pale peach, deeper centre. Dwarf. Garden, bedding, edging, glass. Prune 34 or 42 .

Augustine Guinoisseau (H.T.), Guinoisseau, 1889. -White, tinted blush. Vigorous. Garden, standard, bedding. Free flowering and very fragrant. Sport from "La France." Prune 6.

Augustus Hartmann (H.T.), B. R. Cant \& Sons, 1914.-Brilliant metallic red. Vigorous. Exhibition, bedlding, glass. Prune 2 or 3 .

Austrian Copper (Austrian Briar), grown in England in 1596 by John Gerard. Nasturtium red, reverse of petals yellow. Vigorous. Garden. Single-flowered. Early summer-flowering. Prune 19.

Austrian Yellow (Austrian Briar), grown in England in 1596 by John Gerard. Clear yellow. Vigorous. Garden. Single-flowered. Early summer-flowering. Prune 19. 
Aviateur Bleriot (wich. ramb.), Fauque et Fils, 1910.-Golden yellow. Vigorous climber. Pillar, arch, pergola. One of the best wichuraianas. Prune 37.

Avoca (H.T.), A. Dickson \& Sons, 1907.-Rich crimson. Very vigorous. Exhibition, garden, standard, pillar, pegging-down. Very fragrant. Prune 6 or 7 .

Banksia alba (Banksia), Kerr, 1807.-White, Very vigotous climber. South or West wall. Does not flower freely until well established. Small double white flowers. Knowi as the White Banksian Rose. Summer-flowering. Fragrant. Prune 26.

Banksia lutea (Banksia), Royal Horticultural Society, 1824.-Yellow. Very vigorous. South or West wall. Does not flower freely until well established. Small double yellow flowers. Known as the Yellow Banksian Rose. Summer-flowering. Prune 26.

Benedicte Sequin (H.'T.), Pernet-Ducher, 1919.Coppery orange. Vigorous. Garden, bedding. Prune 4.

Bennett's Seedling (Ayr.), Bennett, 1840.-White. Vigorous climber. Arch, pergola, hedge, N. wall. A very free-flowering summer Rose. Known also as "Thoresbyana." Fragrant. Prune 23 or 39.

Bessie Brown (H.T.), A. Dickson \& Sons, 1899.Creamy white Moderately vigorous. Exhibition. Prune 5.

Betty (H T .), A. Dickson \& Sons, 1905.-Coppery rose, shaded yellow. Vigorous. Grarden, bedding. Very Jong petals. Fragrant. Prune 4.

Blanc double de Coubert (rug.), Cochet-Cochet, 1892.-Pure white. Very vigorous. Bush, hedge, town. Semi-double. Fragrant. Prune 35. 
Blanche Moreau (Moss), Moreau-Robért, 1880.Pure white. Vigorous. Bush. The best white moss. Summer-flowering. Fragrant. Prune 12 (a).

Blush Rambler (mult. ramb.), B. R. Cant \& Sons, 1908.-Blush rose. Very vigorous climber. Pillar, arch, pergola, hedge. One of the best of the ramblers. Flowers in large clusters. Summer-flowering. Best on chalk soil. Prune 27 or 39.

Bouquet d'Or (Dijon Tea), Ducher, 1872._-Buff yellow. Vigorous climber. Standard, pergola, N. wall. Fragrant. Prune 33 or 39.

Canarienvogel (poly. pom.), Welter, 1904.-_Deep golden yellow, shaded crimson. Dwarf. Bedding, edging. The brightest yellow poly. pom. Prune 34 .

Candeur Lyonnaise (H.P.), Croibier, 1913.-Pure white, base of petals sulphur. Very vigorous. Exhibition. Prune 3.

Captain Hayward (H.P.), Bennett, 1893.-Light scarlet crimson. Vigorous. Exhibition, garden, standard. Best in a cool season. Fragrant. Prune 4 or 5.

Carmine Pillar (mult. ramb.), Paul \& Son, 1895.Bright scarlet-lake. Very vigorous. Garden, pillar, arch, pergola. Very early summer-flowering. Singleflowered. Prune 27 or 39.

Caroline Testout (H.T.), Pernet fils-Ducher, 1890. -Bright warm pink. Vigorous. Exhibition, garden, standard, bedding, glass, town. One of the very best for all purposes. Prune 5, 6, or 42.

Caroline Testout, Climbing (H.T.), Chauvry, 1902.-Pillar, arch, pergola, E. wall. A reliable climbing sport from "Caroline Testout." Prune 10 or 39 . 
Cecile Brunner (poly. pom.), Ducher, 1880.Blush white, shaded pale rose. Dwarf. Garden, bedding, edging. One of the best of its class. Prune 34.

Celestial (Alba.), Blush.-Very vigorous. Bush. Glaucous foliage. Summer-flowering. Prune 15.

Charles E. Shea (H.T.), Elisha J. Hicks, 1916.Rich salmon pink. Vigorous. Exhibition, garden, bedding, glass. Very fine. Prune 4 or 42 .

Charles K. Douglas (H.T.), Hugh Dickson, 1919. -Crimson scarlet. Vigorous. Garden, bedding. Promising. Prune 4.

Charlotte Klemm (C.), Turke, 1905.-Fiery red. Moderately vigorous. Garden. A fine China. Prune 31 .

Château de Clos Vougeot (H.T.), Pernet-Ducher, 1908.-Dark crimson, shaded scarlet. Moderately vigorous. Garden, standard, bedding. One of the darkest Roses. Very fragrant. Prune 2.

Chațillon Rambler (wich. ramb.), Nonin, 1913,Delicate pink. Very vigorous. Pillar, arch, pergola. Prune 37.

Cheerful (H.T.), S. McGredy \& Son, 1915.-Pink terra-cotta. Garden. Distinct colour. Prune 4.

Cherry Page (H.T.), W. Easlea, 1914.-Brilliant cherry pink. Very vigorous. Gardien, bedding, deconative. Semi-double. Prune 4.

China, Common (C.), Parsons, 1796.-China pink. Vigorous. Garden, bedding, hedge. Continuous blooming. Known as the "Old Monthly." Prune 31.

Chrissie Mackellar (H.T.), A. Dickson \& Sons, 1913.-Orange pink. Vigorous. Bedding. Very free-flowering, lovely foliage. Semi-double. Prune 4. 
Christine (Pernet), S. McGredy \& Son, 1918.Deep golden yellow. Vigorous, branching. Bedding. Unusually free and keeps its colour. Prune 4.

Christine Wright (wich. ramb.), Hoopes and Thomas, 1903.-Bright pink. Very vigorous. Pillar, arch, pergola. Early. An effective variety. Upright growth. Prune 37.

Clarice Goodacre (H.T.), A. Dickson \& Sons, 1916. - Chrome on ivory white. Vigorous. Exhibition, garden. Good in autumn. Prune 4 or 5.

Clytemnestra (Hybrid Musk), Rev. J. H. Pemberton, 1915.-Salmon-pink. Vigorous. Bedding, bush. Prune 18.

Colonel Oswald Fitzgerald (H.T), A. Dickson and Sons, 1917.-Blood-red velvety crimson. Vigorous. Bedding, glass. Rich colour. Prune 4.

Commandant Beaurepaire (D.), Moreau-Robért, 1875.-Bright rose, striped white. Vigorous. Garden, shrubbery. Prune 14 (a).

Commandant Félix Faure (H.P.), Boutigny, 1902. -Dark crimson, flushed vermilion. Vigorous. Garden, standard, bedding. The colour does not fade. Very fragrant. Prune 6.

Common China.-See "China, Common."

Common Moss.-See " Moss, Common."

Common Provence.-See " Provence, Common."

Common Sweetbriar.-See "Sweetbriar, Common."

Comtesse du Cayla (C.), Guillot, 1902.-Nasturtium red. Vigorous. Garden, standard, bedding, bush. A distinct shade in the China roses. Variable in colour. Prune 31. 
Conrad F. Meyer (rug.), Frobel, 1900.-Clear silvery rose. Very vigorous. Garden, bush, pillar, pergola, hedge, N. wall, town. Flowers early in the summer and again in the autumn. Very fragrant. Prune 35 (33) or 39.

Constance (Pernet), Pernet-Ducher, 1915.-Bright golden and canary yellow. Vigorous. Garden, standard, bedding. Prune 20.

Corallina (T.), W. Paul \& Son, 1900.-Deep coral red. Vigorous. Garden, standard, bedding. Good in autumn. Prune 4.

Coronation (H.P.), Hugh Dickson, 1913.-Pale rose-pink. Vigorous. Exhibition. Large globular bloom. Prune 2 or 3.

Coronation (wich. ramb.), Turner, 1912.-Scarlet crimson, splashed white streaks. Vigorous climber. Pergola. Prune 37 or 39.

Countess of Shaftesbury ( $\mathrm{H} . \mathrm{T}^{\mathrm{T}}$.), Hugh Dickson, 1909.-Silvery carmine, with picotee edging. Moderately vigorous. Exhibition, garden, glass. Prune 4, 5 , or 42 .

Countess of Warwick (H.T.), W. Easlea, 1919.Creamy yellow, edged pink. Vigorous. Garden, bedding. Prune 4.

Covent Garden (H.T.), B. R. Cant \& Sons, 1919. -Deep crimson. Vigorous. Garden, bedding, glass. Good in Autumn. Prune 4 or 42.

Cramoisie Supérieure, Climbing (C.).--Pillar. A climbing sport from " Cramoisie Supérieure." Prune 10 or 31 .

Crested Moss (Moss), Vibert, 1827.-Rosy pink. Vigorous. Bush. Heavily mossed. Summer-flowering. Prune 12. 
Crimson China.-See " Old Ci:mson China."

Crimson Rambler (mult. ramb.), Turner, 1893.Crimson. Vigorous climber. Pillar, arch, pergola. A well-known climber. Late summer-flowering. Rather subject to mildew. Prune 27 or 39.

Cupid (H.T.), B. R. Cant \& Sons, 1915.-Pale peach. Very vigorous. Bush, pillar. Single-flowered. Large bush. Prune 39.

C. V. Haworth (H.T.), A. Dickson \& Sons, 1917. -Scarlet crimson. Moderate. Exhibition, bedding. Fine colour. Prune 4.

Cynthia Forde (H.T.), Hugh Dickson, 1909.Brilliant rose-pink. Vigorous. Exhibition, garden, standard, bedding. Free and branching. A beautiful pink rose. Prune 4 or 5.

Danäe (Hybrid Musk), Rev. J. H. Pemberton, 1913. Soft yellow. Vigorous. Garden, bush, hedge. Perpetual flowering. Flowers in clusters. Semidouble. Prune 18.

Dean Hole (H.T.), A. Dickson \& Sons, 1904-Pale silvery rose, deeper shaded. Vigorous. Exhibition. Fragrant. Prune 5.

Débutante (wich. ramb.), M. H. Walsh, 1902.Light pink. Vigorous climber. Pillar, arch, pergola, weeping standard. Late summer-flowering. Fragrant. Prune 37.

Diabolo (wich. ramb.), Fauque et fils, 1908.Dark glowing crimson. Very vigorous. Pillar, arch, pergola. Large almost single flowers with golden stamens. Upright growth. Summer-flowering. Prune 37.

Donald McDonald (H.T.), A. Dickson \& Sons, 1916.-Orange carmine. Vigorous. Bedding. Prune 6. 
Dorothy Page=Roberts (H.T.), A. Dickson \& Sons, 1907.-Coppery pink. Vigorous. Garden, bedding. A beautiful shade of pink. Fragrant. Prune 4 or 5 .

Dorothy Perkins (wich. ramb.), Jackson \& Perkins, 1901.-Rose pink. Very vigorous climber. Pillar, arch, pergola, creeping, weeping standard, hedge, screen. Almost evergreen. One of the best. Late flowering. Prune 37 or 39.

Dowager Countess of Roden (H.T.), W. Paul \& Son, 1919. - Silvery pink. Moderate. Garden. Prune 4.

Dr. Joseph Drew (H.T.) (Courtney Page), W. Easlea, 1918.- - Yellow, shaded pink. Very vigorous. Exhibition, garden, decorative. Very fragrant. Prune 4 or 5 .

Dr. Van Fleet (wich, ramb.), Van Fleet, 1910.Soft blush. Very vigorous. Pillar, arch, pergola. Flowers in Autumn. Prune 27 or 39.

Duchess of Wellington (H.T.), A. Dickson and Sons, 1909.-Deep saffron yellow, outside petals orange. Vigorous. Garden. Best in dry season. Very fragrant. Prune 4.

Duchess of Westminster (H.T.), A. Dickson and Sons, 1911.-Rose madder. Vigorous. Exhibition, garden, glass. Prune 4, 5, or 42 .

Dundee Rambler (Ayr.), Martin.-White, pink edges. Very vigorous climber. Arch, pergola, hedge, $\mathrm{N}$. wall, town, very exposed gardens. Summerflowering. Prune 23 or 39.

Ecarlate (H.T.), Boytard, 1907.--Light scarlet. Moderately vigorous. Garden, standard, bedding. Very bright and free flowering. Semi-double. Prune 4 . 
Edel (H:T.), S. McGredy \& Son, 1919.-White. Vigorous. Exhibition, garden, glass. Mildew-proof. Prune 4 or 42.

Edgar M. Burnett (H.T.), S. McGredy \& Son, 1914.-Flesh, tinted rose. Moderately vigorous. Exhibition, glass. An exhibitor's Rose. Prune 4, 5, or 42 .

Edith Cavell (H.T.), Chaplin Bros., 1919.Creamy white. Vigorous. Garden, standard, bedding, glass. Prune 4 or 42 .

E. Godfrey Brown (H.T.), Hugh Dickson, 1919.Crimson. Vigorous. Exhibition. Prune 3.

Elegante (H.T.), Pernet-Ducher, 1919.-Orange yellow. Vigorous. Grarden, bedding, glass. Prune 4 or 42 .

Elisa Robichon (wich. ramb.), Barbier, 1901.Rose, shaded pale yellow. Vigorous climber. Pillar, arch, pergola, creeping, weeping standard, hedge. Summer-flowering. Prune 37.

Ellen Poulsen (poly. pom.), Poulsen, 1912.Bright cherry rose. Moderately vigorous. Garden, standard, bedding, glass, decorative. Prune 34 or 42.

Emily Gray (H. wich.) (Dr. Williams), B. R. Cant and Sons, 1916.-Golden yellow. Very vigorous. Pillar, arch, pergola, wall. Prune 37 or 39.

Emma Wright (H.T.), S. McGredy \& Son, 1917.Pure orange. Vigorous. Bedding. Fine colour. Semi-double. Prune 4.

Ethel (wich. ramb.), Turner, 1912._Clear flesh pink. Vigorous climber. Weeping standard. Semidouble. Prune 37. 
Eugénie Lamesch (poly. pom.), P. Lambert, 1899. - Clear yellow, buds edged carmine. Dwarf. Garden, bedding, edging. Distinct violet scent. Prune 34.

Evangeline (wich. ramb.), M. H. Walsh, 1906.Lavender pink with white centre--Very vigorous climber. Arch, pergola, weeping standard. Midseason. Single-flowered. Summer-flowering. Very fragrant. Prune 37 or 39.

Excelsa (wich. ramb.), M. H. Walsh, 1909.Bright rosy crimson. Vigorous climber. Pillar, arch, pergola, creeping, weeping standard. Late summerflowering. Prune 37.

Fabvier (C.), Laffay, 1832.-Dazzling crimson, with white centre. Dwarf: Bedding, edging. One of the best crimson Chinas. Prune 31 .

Fellenberg (C.), Fellenberg, 1857.--Rosy crimson. Vigorous. Garden, bush, hedge, dwarf-wall. Fragrant. Prune 31 (a) or 39.

Félicité $=$ et $=$ Perpétue (Sempervirens), Jacques, 1828. - Creamy white. Very vigorous climber. Arch, pergola, hedge, N. wall, very exposed gardens. A good climbing rose. Early summer-flowering. Prune 24 or 39 .

Flame of Fire (H.T.), S. McGredy \& Son, 1916. -Orange flame. Vigorous. Bedding. Prune 6 or 42.

Flora McIvor (S. Briar) (Lord Penzance), Keynes, Williams \& Co., 1894.-Blush white, tinted rose. Very vigorous. Bush, hedge. Single-flowered. Early summer-flowering. Prune 22 (b).

Florence Forrester (H.T.), S. McGredy \& Son, 1914.-Pure white. Robust. Exhibition. Prune 2 or 3 . 
Florence·H. Veitch (H.T.), W. Paul \& Son, 1911. -Bright scarlet crimson. Vigorous. Semi-climber. Standard, bush, pillar, hedge, E. wall, pegging-down. Very free. Very fragrant. Prune 10 or 39.

Florence Pemberton (H.T.), A. Dickson \& Sons, 1902.-Creamy white, edged blush. Vigorous. Exhibition, standard. Free flowering. Prune 7.

Fortune's Yellow (N.), Fortune, 1845.-Orange yellow, shaded and flaked metallic red. Vigorous climber. South or west wall. Best under glass. Also known as " Beauty of Glazenwood." Summer-flowering. Prune 32.

Frances Gaunt (H.T.), A. Dickson \& Sons, 1918. -Fawn apricot. Vigorous. Bedding, decorative. Prune 4.

François Crousse (H.T.), Guillot, 1900.-Crimson. Vigorous. Pillar, wall. A fine crimson pillar rose. Good early and late. Prune 10 or 39.

François Guillot (wich. ramb.), Barbier, 1905.Yellow, white, and pink. Vigorous. Pillar, arch, pergola. Prune 37.

François Juranville (wich. ramb.), Barbier, 1906. -Deep fawn pink. Very vigorous. Arch, pergola, weeping standard, hedge. Very fragrant. Early summer-flowering. Prune 37 or 39.

Frau Karl Druschki (H.P.), P. Lambert, 1900.Pure white. Very vigorous. Exhibition, garden, standard, bedding, pillar, glass, town, pegging down. The best white rose for all purposes, but lacking in fragrance. It may be grown as a semi-climber. Prune $6,7,41$, or 42 .

Gardenia (wich. ramb.), Manda, 1899.-Bright yellow, changing to cream. Very vigorous climber. Pillar, arch, pergola, creeping, screen. One of the best yellow wichuraianas. Early summer-flowering. Prune 37 or 39. 
G. C. Waud (H.T.), A. Dickson \& Sons, 1908.Rose, suffused orange and scarlet. Moderately vigorous. Exhibition, garden, bedding. A most distinct shade of colour. Tea scented. Prune 2 or 3.

Général Jacqueminot (H.P.), Roussel, 1853.Bright scarlet crimson. Vigorous. Garden, standard. Free flowering. Very fragrant. Prune 4.

General McArthur (H.T.), The E. G. Hill Co., 1905.-Bright scarlet crimson. Vigorous. Garden, standard, bedding, town. A fine bedding Rose. Very fragrant. Prune 4.

George Dickson (H.T.), A. Dickson \& Sons, 1912. - Deep velvety crimson, heavily veined. Vigorous. Exhibition, standard. A grand dark crimson. Very fragrant. Prune 4 or 5.

George Elgar (poly. pom.), E. Turbat, 1912.-Coppery yellow, passing to clear yellow. Dwarf. Bedding. Prune 34.

Gerbe Rose (wich. ramb.), Barbier, 1905.-Deep pink. Very vigorous. Pillar, pergola. Large bush. Iovely foliage. Fragrant. Prune 37 or 39.

Gladys Holland (H.T.), S. McGredy \& Son, 1916. -Buff yellow and apricot. Moderate. Exhibition, bedding, glass. Prune 4,5 , or 42 .

Gloire de Chédane=Guinoisseau (H.P.), ChédaneGuinoisseau, 1907.-Crimson. Vigorous. Exhibition. Prune 6 or 7.

Gloire de Dijon ( $T$.), Jacotot, 1850.-Buff, or salmon yellow. Vigorous climber. Garden, standard, bush, pergola, town, pegging down, $\mathrm{N}$. and $\mathrm{E}$. walls. A good old climbing rose. Very fragant. Prune 33 or 39 . 
G. Nabonnand (T.), Nabonnand, 1889.-Pale Hlesh, shaded rose. Vigorous. Garden, standard, bedding. Good in autumn. Fragrant. Prune 6.

Golden Emblem (Pernet), S. McGredy \& Son, 1916.-Grolden yellow. Vigorous. Standard, bedding, glass. A very fine Rose. Prune 20.

Golden Ophelia (H.T.), B. R. Cant \& Sons, 1917. -Yellow, edges of petals cream. Vigorous. Bedding. Prune 4.

Golden Spray (H.T.), Hugh Dickson, 1916.Rich golden yellow. Vigorous. Low walls. Very lovely semi-single flowers. Prune 4.

Goldfinch (mult. ramb.), Paul \& Son, 1908.Deep yellow in bud, changing to cream. Vigorous climber. Pillar, arch, pergola. Early summerflowering. Prune 27.

Gorgeous (H.T.), Hugh Dickson, 1915.-Orange yellow, flushed reddish copper. Vigorous. Exhibition, garden, standard, bedding, glass. Prune 4, 5, or 42 .

Gruss an Teplitz (H.T.), Geschwind, 1897.Bright crimson. Very vigorous. Garden, standard, bedding, bush, pillar, E. wall, hedge, pegging down, town. One of the best roses for general garden purposes. A grand autumn rose. Fragrant. Prune 10,39 , or 41 .

Gustav Grünerwald (H.T.), P. Lambert, 1903.Carmine pink, with yellow base. Vigorous. Garden, standard, bedding, town. A good all-round rose. Very fragrant. Prune 6.

Gustave Régis (H.T.), Pernet fils-Ducher, 1890. -Nankeen yellow. Very vigorous. Garden, standard, bush, pillar, pegging down. One of the best buttonhole roses. Prune 39 or 41 . 
Hadley (H.T.), Waban Conservatories, 1914.Dark velvety crimson. Vigorous. Bedding, standard, glass. Fragrant. Prune 4 or 42.

Harrisonii (Austrian Briar), Harrison, 1830.--Soft golden yellow. Vigorous. Garden, bush, dwarf hedge. Semi-double. Summer-flowering. Prune 19.

H. D. M. Barton (H.T.), Hugh Dickson, 1917.Velvety crimson. Vigorous. Garden, bedding. Showy. Prune 4.

Hebe's Lip (Species). - White, with picotee edge of purple. Vigorous. Bush, hedge. Semi-double. Summer-flowering. Prune 28.

Helen Chamberlain (H.T.), W. Easlea, 1917.Creamy yellow, shaded orange. Moderate. Exhibition, bedding. Fine large petals. Prune 5.

Henrietta (H.T.), Merryweather, 1917.-Fiery orange crimson. Vigorous. Garden, bedding. Semi-double. Prune 4.

Hiawatha (wich. ramb.), M. H. Walsh, 1905.Rich crimson with white eye. Very vigorous climber. Pillar, arch, pergola, weeping standard, screen. Single-flowered. Late summer-flowering. Prune 37 or 39 .

Hoosier Beauty (H.T.), Dorner \& Sons, 1915.Fiery crimson. Vigorous. Garden, bedding, glass. Prune 4 or 42.

Horace Vernet (H.P.), J. B. Guillot, fils, 1866 .Scarlet-crimson, dark shaded. Moderate. Exhibition. Fragrant. Prune 3.

H. P. Pinkerton (H.'T.), Hugh Dickson, 1916.Brilliant scarlet. Vigorous. Exhibition, bedding. Prune 5. 
Hugh Dicksion (H.P.), Hugh Dickson, 1904.Crimson, shaded scarlet. Very vigorous. Exhibition, garden, standard, bedding, pillar, hedge, town, pegging down. Very fragrant. Prune 6, 7, or 41 .

Hugonis (Species), J. Veitch \& Sons, 1908.Sulphur-yellow. Very vigorous. Bush. Singleflowered. Very early. Summer-flowering. Prune 28.

H. V. Machin (H.T.), A. Dickson \& Sons, 1914. Scarlet crimson. Moderately vigorous. Exhibition. Prune 3.

Independence Day (Pernet), Bees, Ltd., 1919.Deep orange. Vigorous. Bedding, glass. Delightful bud. Prune 4 .

Irish Afterglow (H.T.), A. Dickson \& Sons, 1917. -Tangerine passing to pink. Vigorous. Bedding. A sport from Irish Elegance. Single-flowered. Prune 4.

Irish Elegance (H.T.), A. Dickson \& Sons, 1905. -Shades of apricot, buds orange-scarlet. Vigorous. Garden, bedding, bush. Ore of the best singleflowering roses. Prune 4.

Irish Fireflame (H.T.), A. Dickson \& Sons, 1913. -Garden, bedding. A more brilliant "Irish Elegance." Single-flowered. A fine decorative rose. Prune 4.

Irish Fireflame, Climbing (H.T.), A. Dickson and Sons, 1916.-Fiery orange. Very vigorous. Wall. , Fine colour. Single flowered. Prune 10.

Isobel (Pernet), S. McGredy \& Son, 1916.Orange scarlet. Vigorous. Garden, standard, bedding, decorative. Single-flowered. Prune 4.

Janet's Pride (S. Briar), Paul \& Son, 1892.White, shaded and tipped crimson. Very vigorous. Bush, hedge. Early. Semi-single. Early summerflowering. Prune 22 (b). 
J. B. Clark (H.'T.), Hugh Dickson, 1905. Scarletcrimson, shaded black. Very vigorous. Exhibition, garden, standard, bush, pillar, hedge, pegging down, wall, town. Prune 6,7 or 41 .

Jean Guichard (wich. ramb.), Barbier, 1905.Coppery carmine, opening to salmon and rose. Very vigorous climber. Pillar, arch, pergola, creeping, weeping standard. Early summer-flowering. Prune 37 or 39 .

Jeanie Deans (S. Briar) (Lord Penzance), Keynes, Williams \& Co., 1895.--Rosy crimson. Very vigorous. Garden, bush, hedge. Early summer-flowering. Prune 22 (b).

Jessie (poly. pom.), H. Merryweather \& Sons, 1909. - Bright crimson-scarlet. Dwarf. Garden, halfstandard, bedding, edging. Continuous flowering. Prune 34.

Jersey Beauty (wich. ramb.), Manda, 1899.-Rich yellow, opening to cream. Very vigorous climber. Garden, pillar, arch, pergola, creeping, heđge, screen. Single-flowered. Early summer-flowering. Evergreen. Prune 37 or 39 .

Joanna Bridge (H.T.), Elisha J. Hicks, 1916.Pale straw. Vigorous. Garden, bedding. Semisingle. Prune 4.

Jonkheer J. L. Mock (H.T.), Leenders \& Co., 1909.-Deep rose, carmine reverse. Vigorous. Exhibition, garden. Very fragrant. Prune 4 or 5.

Joseph Billard (wich. ramb.), Barbier, 1905.Crimson, centre rich yellow, fading to cream. Vigorous. Garden, pillar, weeping standard. Singleflowered. Early flowering and sometimes flowers again in autumn. Prune 37. 
Joseph Hill (H.T.), Pernet-Ducher, 1903.-Coppery yellow, shaded salmon pink. Vigorous. Exhibition, garden, standard, bedding. Beautiful colour and fine foliage. Fragrant. Prune 4.

Joseph Lamy (wich. ramb.), Barbier, 1906.White, edged mauve. Vigorous climber. Pillar, pergola. Rather scanty in foliage. Summer-flowering. Prune 37 or 39.

Julia Mannering (S. Briar) (Lord Penzance), Keynes, Williams and Co., 1895.-Pearly pink. Very vigorous. Garden, bush, hedge. Early summerflowering. Prune 22 (b).

Katharine Zeimet (poly. pom.), P. Lambert, 1901. -Pure white. Dwarf. Garden, standard, bedding, edging. A pretty, free flowering variety. The best white polyantha. Fragrant. Prune 34.

K. of K. (H.T.), A. Dickson \& Sons, 1917.Brilliant scarlet crimson. Vigorous. Garden, bedding, standard. Semi-single. Prune 4.

Kootenay (H.T.). A. Dickson \& Sons, 1917.-Pale lemon. Vigorous. Exhibition, garden. Prune 3.

Lady Alice Stanley (H.T.), S. McGredy \& Son, 1909.-Silvery pink, reverse of petals rose. Vigorous. Exhibition, garden, bedding, glass. Very fragrant. Prune 4 , 5, or 42

Lady Ashtown (H.T.), A. Dickson d Sons, 1904. -Pure deep pink. Vigorous. Exhibition, garden, standard, bedding. Free flowering. A useful rose. Prune 4 or 5.

Lady Ashtown, Climbing (H.T.), F. M. Bradley, 1909. - S. or W. wall. A climbing sport from " Lady Ashtown." Prune 10. 
Lady Curzon (D.), Turner, 1902.-Pale pink. Vigorous. Bush, pillar, hedge. A good Hybrid Damask. Single-flowered. Early summer-flowering. Prune 14 (a) or 39.

Lady Gay (wich. ramb.), M. H. Walsh, 1905.Rose pink. Very vigorous climber. Pillar, arch, pergola, creeping, weeping standard, hedge, screen. Very much like "Dorothy Perkins," which see. Prune 37 or 39 .

Lady Godiva (wich. ramb.), Paul \& Son, 1908.Pale blush, deeper centre. Vigorous climber. Pillar, arch, pergola, weeping standard. Late flowering. Almost evergreen. A sport from "Dotothy Perkins." Prune 37 or 39 .

Lady Greenall (H.T.), A. Dickson \& Sons, 1911. -Saffron-orange, bordere'd cream. Vigorous. Garden, glass. Fragrant. Prune 2 or 42.

Lady Hillingdon (T.), Lowe \& Shawyer, 1910.Bright golden yellow, shaded fawn. Vigorous. Garden, standard, bedding, glass. One of the best yellow roses. A good decorative and buttonhole rose. Prune 4 or 42 .

Lady Hillingdon, Climbing ( $\mathrm{H} . \mathrm{T}$.), Elisha J. Hicks, 1917.-Bright golden yellow. Very vigorous. $\therefore$. or W. wall. A climbing sport from "Lady Hillingdon" ; good. Prune 10.

Lady Penzance (S. Briar) ( $\mathrm{L}$,ord Penzance), Keynes, Williams and Co., 1894.-Coppery yellow. Vigorous. Garden, bush, hedge. The most distinct Penzance briar. Single-flowered. Early summerflowering. Prune 22 (b).

Lady Pirrie (H.T.), Hugh Dickson, 1910.-Delicate coppery salmon. Vigorous. Garden, standard, bedding, glass. A delightful combination of colour. A fine decorative rose. Prune 4. 
Lady Plymouth ('T.), A. Dickson \& Sons, 1914.Ivory cream, flushed pink. Vigorous. Exhibition. Prune 2 or 3.

Lady Roberts (T.), F. Cant \& Co., 1902.Apricot, with coppery yellow base. Vigorous. Exhibition, garden, standard, bedding, glass. A good buttonhole rose. Fine under glass. A sport from "Anna Olivier." Prune 4, 5, or 42.

Lady Waterlow (H.T.), Nabonnand, 1903.-Pale salmon blush, edged carmine. Semi-climber. Garden, standard, bush, pillar, pegging down. E. wall. A fine pillar rose. Semi-double. Prune 6,39 , or 41 .

La France (H.T.), J. B. Guillot, fils, 1867.Silvery rose, with pale lilac shading. Vigorous. Garden, standard, bedding, glass. Very fragrant. Prune 6, 7, or 42 .

Lamia (H.T.), W. Easlea, 1918.-Reddish orange. Vigorous. Bedding. A fine novelty. Semi-double. Prune 4.

La Tosca (H.T.), V. Schwartz, 1900.-Pale blusb white, rose centre. Vigorous. Garden, standard. bedding, bush, town. Good in autumn. Prune 6 .

Laurent Carle (H.T.)., Pernet-Ducher, 1907.-Deep carmine. Vigorous. Garden, standard. Very fragrant. Prune 4.

Laurette Messimy (C.), Guillot, 1887.-Pale rose, with yellow base. Vigorous. Garden, standard, bedding, bush. Fine for massing. Prune 31.

Lemon Pillar (Paul's) (H.N.), Paul \& Son, 1915. -Sulphur yellow. Very vigorous. Pillar. Prune 18 or 39

Léonie Lamesch (poly. pom.), Lambert, 1899.Bright coppery red, with golden centre. Vigorous Garden, standard, bush. Fragrant. Prune 34. 
Léontine Gervais (wich. ramb.), Barbier, 1906.Salmon rose, tinted yellow. Vigorous climber. Pillar, arch, pergola. A fine variety. Early summerflowering. Very fragrant. Prune 37 or 39.

Liberty (H.T.), A. Dickson \& Sons, 1900.-Crimson. Maderately vigorous. Garden, bedding, glass. Well-shaped bloom. A good buttonhole rose. Very fragrant. Prune 4 or 42 .

Lieutenant Chauré (H.T.), Pernet-Ducher, 1910. -Rich crimson. Vigorous. Exhibition, garden, bedding, glass. Fine foliage. Keeps its colour well. Very fragrant. Prune 4 or 42.

Lilian Moore (H.T.), Hugh Dickson, 1916.Pure deep Indian yellow. Moderate. Bedding. Prune 4 or 5.

Little Meg (poly. pom.), W. Easlea, 1917.-Pure white. Vigorous. Bedding. Pretty in the bud. Prune 34.

Lord Penzance (S. Briar), (Lord Penzance), Keynes, Williams \& Co., 1894.-Fawn. Vigorous. Garden, bush, pillar, hed'ge, shrub. Single-flowered. Summer-flowering. Prune 22 (b).

Los Angeles (H.T.), Howard \& Smith, 1916.Salmon rose, shaded apricot. Vigorous. Garden, bedding, glass. Very fine. Fragrant. Prune 4 or 42.

Louise Baldwin (H.T.), S. McGredy \& Son, 1919. -Golden yellow. Vigorous. Bedding, glass. Prune + or 42 .

Louise Catherine Breslau (Pernet), PernetDucher, 1912.-Salmon pink, suffused copper. Vigorous. Standard, garden, bedding. Prune 20.

Louise Crette (H.F.), Chambard, 1915.-White. Vigorous. Exhibition, standard. Very fine. Prune 
lucida (Species), N. America, 1724.-Bright rose pink. Vigorous. Garden, bush, hedge. Red foliage in autumn. Single-flowered. Summer-flowering. Prune 28.

lucida plena (Species), Veitch, 1884.-Rose. Vigorous. Garden, bush, hedge. A double form of the above. A pretty buttonhole variety. Summerflowering. Prune 28.

Lyon Rose (Pernet), Pernęt-Ducher, 1907.Salmon rose, suffused yellow. Moderately vigorous. Exhibition, standard, glass. Fragrant. Prune 5, 20, or 42 .

Mabel Drew (H.T.), A. Dickson \& Sons, 1911.Creamy yellow. Vigorous. Exhibition. Fragrant. Prune 4 or 5 .

macrantha (Hybrid of Species).-Flesh. Climber. Bush, pillar. One of the best of the single-flowered roses. Mid-season. Summer-flowering. Prune 29 (14) or 39.

Mme. Abel Chatenay (H.T.), Pernet-Ducher, 1895.- Pale salmon pink with deeper centre. Vigorous. Garden, standard, bedding, glass, town. One of the best garden roses. Continuous flowering. A good buttonhole rose. Very fragrant. Prune 4 or 42.

Mme. Abel Chatenay, climbing (H.T.), W. Easlea, 1916.-Pale salmon pink, with deeper centre. Tery vigorous. Pillar, arch, low wall. A very fine Rose. Very fragrant. A reliable sport from "Mme. Abel Chatenar." Prune 10.

Mme. Alfred Carrière (H.N.), Schwartz, 1879.White, shaded blush. Vigorous climber. Pillar, arch, pergola, E. wall. The best white hardy climber. Flowers again in autumn. Very fragrant. Prune 18 or 39 
Mme. Antoine Mari ( $T$.), A. Mari, 1902.-Rose, shaded white, opening blush-white. Vigorous. Garden, standard, bed'ding. Foliage almost evergreen. Mildew proof. Good in autumn. Prune 6.

Mme. Constant Soupert (T.), Soupert et Notting, i905.-Deep yellow, shaded peach. Moderately vigorous. Exhibition, glass. A fine Tea in a dry season. Prune 2, 3, or 42 .

Mme. C. Martel (H.T.), Pernet-Ducher, 1917.Sulphur yellow. Exhibition. Fragrant. Prune 3.

Mme. d'Arblay (Hybrid Musk).-Flesh, changing to white. Very vigorous. Pillar, arch, pergola. Summer-flowering. Fragrant. Prune 18 or 39.

Mme. Edouard Herriot (Pernet), Pernet-Ducher, 1913.-Vivid terra-cotta, passing to clear strawberry rose. Semi-double. Garden, standard, bedding, -glass, town. Glossy green folliage. The "Daily Mail" Rose. Prune 20.

Mme. Eugène Resal (C.), Guillot, 1895.-Coppery rose, shaded orange. Vigorous. Garden, standard, bedding, bush. Striking colour. Prune 31.

Mme. Jean Dupuy ('T.), P. Lambert, 1902.Yellow, edged rose. Vigorous. Garden, standlard. A good buttonhole Rose. Prune 6 .

Mme. Jules Bouché (H.T.), Croibier, 1911.-Porcelain white. Vigorous. Garden, bedding. Prune 4.

Mme. Jules Gouchault (poly. pom.), Turbat, 1914. -Orange pink. Vigorous. Bedding. Prune 34.

Mme. Jules Gravereaux ( $T$.), Soupert et Notting, 1901.-Flesh, shaded yellow. Very vigorous. Exhibition, pegging down, wall. Free-flowering. Prune 6, 7 , or 41 . 
Mme. Jules Grolez (H.T.), Guillot, 1897.-Bright silvery rose. Moderately vigorous. Garden, standard, bedding. A good and distinct garden rose. Rather subject to mildew. Fragrant. Prune 4.

Mme. Léon Pain (H.T.), Guillot, 1904.-Silvery flesh. Vigorous. Garden, standard, bedding, town. A good garden Rose. Prune 4.

Mme. Ravary (H.T.), Pernet-Ducher, 1899.-Pale orange yellow, deeper centre. Vigorous. Garden, standiard, bedding, glass, town. A good bedding rose. Prune 4 or 42 .

Mme. Segond=Weber (H.T.), Soupert et Notting, 1908.-Light rose salmon, deeper centre. Vigorous. Garden, standard, bedding, glass. A good bedding rose. Prune 4 or 42.

Maiden's Blush (Alba), 1797.-Blush, deeper centre. Vigorous. Garden, bush. Hardy and fragrant. Summer-flowering. Prune 15.

Maman Cochet (T.), Cochet, 1893.-Deep flesh, shaded fawn, outer petals suffused light rose. Vigorous. Exhibition, garden, standard. One of the best and most reliable of the Teas. Prune 6,7 , or 42 .

Maman Turbat (poly. pom.), Turbat \& Co., 1911. -Peach pink. Bed'ding, edging. Very free-flowering. Prune 34.

Marcella (H.T.), W. Paul \& Son, 1913.-Salmon buff. Exhibition, glass. Prune 4, 5, or 42 .

Marchioness of Ormonde (H.T.), Hugh Dickson, 1918. Straw yellow. Moderately vigorous. Exhibition. Prune 3.

Maréchal Niel (N.), Pradel, 1864.--Deep bright golden yellow. Vigorous climber. Exhibition, standard, S. or W. wall, glass. Best under glass. Requires a warm situation out of doors. Tery fragrant. Prune 32 or 42. 
Margaret (H.T.), W. Paul \& Son, 1909.-Soft salmon-rose. Vigotous. Exhibition, garden, glass. Prune 4, 5, or 42.

Margaret Dickson Hamill (H.T.), A. Dickson and Sons, 1915.-Pale straw. Vigorous. Garden, standard, bedding. A very fine Rose. Prune 4.

Marie Pavie (poly. pom.), Alégatière, 1888.-Pale flesh, rose tinted. Vigorous. Garden, bedding. Prune 34.

Marie van Houtte (T.), Ducher, 1871.-Lemon yellow, petals edged with rose. Vigorous. Garden, standard, bedding. A reliable Tea. Good on a dwarf sunny wall. Fragrant. Prune 6.

May Marriott (Pernet), T. Robinson, 1917.Orange red. Vigorous. Garden, bedding. Prune 20.

Medea (T.), W. Paul \& Son, 1891.-Lemon yellow. Moderate. Exhibition. Best in warm weather. Prune 5.

Meg Merrilies (S. Briar) (Lotd Penzance), Keynes, Williams and Co., 1893.-Bright crimson. Very vigorous. Bush, pillar, hedge. One of the best of its class. Single-flowered. Early summer-flowering. Prune 22 (b).

Mélanie Soupert (H.T.), Pernet-Ducher, 1905.Pale yellow, suffused amethyst. Vigorous. Exhibition, garden, standard, bedding, glass. A refined and beautiful colour. Best in a cool season. One of the most beautiful of all Roses. Prune 4, 5, or 42.

Mélanie Soupert, Climbing (H.T.), J. Burrell and Co., 1914.-Pale yellow, suffused amethyst. Very vigorous. Pillar, arch, $\mathrm{S}$ or $\mathrm{W}$. wall. A reliable free flowering climber. Prune 10 or 39. 
Melody (H.T.), A. Dickson \& Sons, 1911.-Deep saffron yellow, with primrose border. Moderate. Best under glass. Fragrant. Prune 42.

Mermaid (Hy. Brac.), W. Paul \& Son, 1917.Pale sulphur yellow. Vigorous. Garden, bedding, wall, fence, bush. Lovely large single blooms. Hardy. A very fine rose. Prune 44.

Mildred Grant (H.T.), A. Dickson \& Sons, 1901. -Ivory white, tinted peach. Robust. Exhibition. A fine exhibition variety. Prune 3.

Minnehaha (wich. ramb.), Walsh, 1905.-Deep pink. Very vigorous climber. Pillar, arch, pergola, weeping standard. An exceptionally large truss. Rosette flowers. Late summer-flowering. Prune 37 or 39 .

Miriam (H.T.), Rev. J. H. Pemberton, 1919.Capucine. Vigorous. Bedding. Prune 4.

Miss Willmott (H.T.), S. McGredy \& Son, 1916. Soft creamy white. Vigorous. Exhibition, glass. Very lovely. Prune 4 or 42.

Modesty (H.T.), S. McGredy \& Son, 1916.Pearly white, shaded rose. Vigorous. Exhibition, garden, standard. Prune 4 or 5.

Molly Bligh (H.T.), A. Dickson \& Sons, 1917.Orange pink. Vigorous. Exhibition. Fragrant. Prune 3.

Molly Sharman=Crawiord (T.), A. Dickson and Sons, 1908.-White, shaded eau-de-nil. Vigorous. Exhibition, garden, standard, bedding, glass. A very good Tea. Prune 4, 5, or 42 .

Moonlight (Hybrid Musk), Rev. J. H. Pemberton, 1913.-Lemon white. Garden, bush, hedge. Perpetual-flowering. Flowers in clusters. Semi-single. Prune 18. 
moschata (Species).-White. Very vigorous climber. Arch, pergola. The wild briar of the Himalayas. Bears large clusters of single white flowers. Summer-flowering. Fragrant. Prune 28 or 39.

moschata alba (Hybrid of Species).-White, with yellow stamens. Vigorous climber. Garden. Bears clusters of large single white flowers. Prune 29.

moschata fl. pl. (Hybrid of Species).-White Vigorous climber. Arch, pergola. A double-flowered variety of " moschata." Summer-flowering. Prune 29 or 39.

moschata himalayica (Hybrid of Species), Rev. Prebendary Earle.-White, with yellow stamens. Very vigorous climber. Arch, pergola. A rampant climber. Single-flowered. Summer- flowering. Prune 29 or 39 .

Mos,s, Common (Moss), 1596.-Pale rose. Vigorous. Garden, bush. The best pink moss. Summerflowering. Prune 12 (a).

\section{Moss, Crested.-See "Crested Moss."}

Moss, Perpetual White (Perpetual Moss), Laffay. -Pure white. Vigorous. Garden, bush. A good white moss. Summer-flowering. Prune 12 (b).

Moyesii (Species), J. Veitch \& Sons, 1909.Brownish scarlet. Bush. Distinct in flower and foliage. Single-flowered. Unique in colour. Summerflowering. Prune 28.

Mrs. Aaron Ward (H.T.), Pernet-Ducher, 1907.Indian yellow, edge of petals white. Vigorous. Garden, bedding. Prune 4.

Mrs. A. E. Coxhead (H.T.), S. McGredy \& Son, 1910.-Claret red. Vigorous. Exhibition, garden, standard. Good in autumn. Very fragrant. Prune 2 or 3. 
Mrs. Alfred Tate (H.T.), S. McGredy \& Son, 1909. - Coppery salmon, shaded fawn. Vigorous. Garden. A fine semi-double decorative Rose. Prune 4.

Mrs. Ambrose Ricardo (Pernet), S. McGredy and Son, 1914.--Honey yellow. Vigorous. Garden, bedding. Prune 20.

Mrs. Bertram J. Walker (H.T.), Hugh Dickson, 1915.-Cerise pink. Vigorous. Garden, bedding. Sweetly perfumed. Prune 4.

Mrs. Bryce Allan (H.T.), A. Dickson \& Sons, 1916.-Coppery pink. Vigorous. Exhibition, garden. Very fragrant. Prune 4 or 5.

Mrs. Campbell Hall (T.) (Dr. Campbell Hall), A. Dickson \& Șons.-Vigorous. Exhibition. Prune 5 .

Mrs. C. Russell (H.T.), Waban Conservatories, 1913.-Rosy carmine, deeper centre. Vigorous. Exhibition, garden, glass. Prune 2, 3, or 42.

Mrs. C. V. Haworth (H.T.), A. Dickson \& Sons, 1919.-Crimson orange. Vigorous. Bedding. Semidouble. Prune 4.

Mrs. Dunbar=Buller (H.T.), A. Dickson \& Sons, 1919.-Rosy carmine. Vigorous. Bedding. Prune 4.

Mrs. Dunlop Best (H.T.), Elisha J. Hicks, 1916.Coppery bronze. Vigorous. Garden, bedding. A fine rose. Prune 6.

Mrs. E. G. Hill (H.T.), Soupert et Notting, 1906. -Pale pink, with coral-red reverse. Vigorous. Garden, bedding. An erect growing "Grand Duc A. de Luxembourg." Prune 4. 
Mrs. Elisha J. Hicks (H.T.), Elisha J. Hicks, 1919.-Flesh. Moderate. Exhibition, glass. Fragrant. Prune 3.

Mrs. E. Powell (H.T.), Bernaix, 1911.-Scarlet, shaded purplish crimson. Vigorous. Garden, standard, bediding, town. Deep purple-tinted foliage. A fine bedding variety. Fragrant. Prune 4.

Mrs, Farmer (Pernet), Pernet-Ducher, 1919.Orange yellow. Vigorous. Bedding. Prune 4.

Mrs. Foley Hobbs (T.), A. Dickson \& Sons, 1910. -Ivory white. Vigorous. Exhibition, standard. A grand Tea. Prune 5.

Mrs. Franklin Dennison (H.T.), S. McGredy and Son, 1915.-Porcelain white. Vigorous. Exhibition. Prune 5.

Mrs. George Norwood (H.T.), Elisha J. Hicks, 1914.-Bright rich pink. Moderately vigorous. Exhibition. Very fragrant. Ptune 3.

Mrs. George Shawyer (H.T.), Lowe \& Shawyer, 1911.-Pale rose. Vigorous. Garden, standard, bedding, glass. Prune 4 or 42 .

Mrs. G. Marriott (H.T.), S. McGredy \& Son,. 1917.-Cream, suffused pink. Vigorous. Exhibition,. garden. Very large, Prune 4 or 5.

Mrs. Henry Morse (H.T.), S. McGredy \& Son,. 1919.-Silvery rose pink. Vigorous. Exhibition, garden, standlard, bediding, glass. A very fine Rose. Prune 3 or 42.

Mrs. H. D. Greene (H.T.), W. Easlea, 1917.Coppery orange. Vigorous. Bedding, glass. Prune 42. 
Mrs. Herbert Stevens (T.), S. McGredy \& Son, 1910.-White. Vigorous. Garden, bedding, standard, glass. A good buttonhole rose. Pointed flowers. Hardy. I beautiful decorative rose. Fragrant. Prune \pm or 42 .

Mrs. J. H. Welch (H.T.), S. McGredy \& Son, 1911.-Rose pink. Vigorous. Exhibition. Semidouble. Prune 3.

Mrs. John Laing (H.P.), Bennett, 1887.-Rosypink. Vigorous. Exhibition, garden, standard, bedding, glass, town. One of the finest all-round H.P.'s. Very fragrant. Prune 4, 5, or 42.

Mrs. Mackellar (H.T.), A. Dickson \& Sons, 1915. -Citron to pale primrose. Vigorous. Exhibition, garden. Prune 4 or 5 .

Mrs. Mona Hunțing (H.T.), Hugh Dickson, 1916. -Chamois yellow. Vigorous. Garden, bedding. Prune 4.

Mrs. O. G. Orpen (D.), (O. G. Orpen), B. R. Cant \& Sons, 1906.--Pale rosy pink. Vigorous semiclimber. Bush, pillar. Subject to mildew. Singleflowered. Summer-flowering. Prune 14 (a) or 39.

Mrs. R. D. McClure (H.T.), Hugh Dickson, 1913. Clear pink. Vigorous. Exhibition. Prune 5.

Mrs. Rediord (H.T.), S. McGredy \& Son, 1919. -Apricot orange. Vigorous. Garden, standard, bedding. Prune 4.

Mrs. Rosalie Wrinch (H.T.), W. \& J. Brown, 1915.-Shell pink. Very vigorous. Pillar. Semisingle. Prune 10.

Mrs. Theodore Roosevelt (H.T.), The E. G. Hill Co., 1903.-Flesh, tinted pink. Moderate. Exhibition. Prune 5. 
Mrs. Wakefield Christie=Miller (H.T.)., S. McGredy \& Son, 1909.-Soft blush, outside of petals deep rose. Vigorous. Garden, bedding. Good in autumn. Prune 4.

Mrs. Wemyss Quin (Pernet), A. Dickson \& Sous, 1914.-Canary yellow. Vigorous. Garden, standard, bedding, decorative. A very fine Rose. Prune 20.

Mrs. W. H. Cutbush (poly. pom.), W. Cutbush and Son, 1906.-.Bright deep pink. Dwarf. Garden, standard, bedding, edging, glass. Very continuous bloomer. One of the best pink varieties of its class. Prune 34 or 42 .

Mrs. W. H. Cutbush, Climbing (poly. pom.).Very vigorous. Pillar, arch. A climbing sport from “Mrs. W. H. Cutbush." Prune 34 or 39.

Mrs. W. J. Grant, Climbing (H.T.), The E. G. Hill Co., 1899.--Pillar, glass, wall. A fine climbing sport of "Mrs. W. J. Grant." Good in autumn. Prune 10,39 , or 42 .

multiflora (Species), from Japan, 1781.-Pure white. Very vigorous climber. Arch, pergola. Large bunches of small white single flowers. Known also as "polyantha simplex." Early summer-flowering. Prune $28(27 a)$ or 39.

multiflora grandiflora (Hybrid of Species), Bernaix, 1886.- Pure white. Very vigorous climber. Arch, pergola. A large flowered variety of "multiflora." Single-flowered. Known also as " polyantha grandiflora." Early summer-flowering. Prune 29 (27), or 39.

Nellie Parker (H.T.), Hugh Dickson, 1916.Creamy white, shaded copper. Vigorous. Garden, bedding. Prune 4. 
Niphetos, Climbing ( $T$.), Keynes, Williams and Co., 1889.-White. Vigorous climber. A fine greenhouse climber. Prune 10.

Noblesse (H.T.), S. McGredy \& Son, 1917.Primrose yellow. Vigorous. Garden, bedding. Prune 4.

Nova Zembla (rug.), Mees, 1907.-Pale silvery rose. Very vigorous. Garden, standard, shrubbery. Prune 35.

Old Crimson China (C.), T. Evans, 1810.-Deep velvety crimson. Moderate. Garden, edging. Prune 31.

Old Gold (H.T.), S. McGredy \& Son, 1913.—Old gold, tinted scarlet. Moderately vigorous. Garden, standard, decorative. Semi-double. A good decorative Rose. Prune 4.

Ophelia (H.T.), W. Paul \& Son, 1912.-Salmon flesh. Vigorous. Garden, standard, bedding, glass. A very fine Rose. Very fragrant. Prune 4 or 42 .

Orleans Rose (poly. pom.), Levavasseur, 1909.Vivid rosy crimson. Bushy. Garden, standard, bedding. Large truss, very free, good foliage. Perpetual flowering. One of the best dwarf polyanthas. Prune 34.

Orleans Rose, Climbing (poly. pom.), Levavasseur \& fils, 1913.-Vigorous. Pillar, arch. A climbing sport from "Orleans Rose." Prune 34.

Papa Gontier, Climbing (H.T.), Cherrier, 1904.Rosy crimson. Vigorous. S. or W. wall. A climbing sport from "Papa Gontier." Prune 10.

Paul Lédé, Climbing (H.T.), 1913.-Very vigorous. Pillar, S. or W. wall. A climbing sport from "Paul Lédé." Prune 10 or 39. 
Pax (Hybrid Musk), Rev. J. H. Pemberton, 1918.-Pure white. Vigorous. Bedding, bush. Very free flowering. A magnificent Rose. Prune 18.

Perle d'Or (poly. pom.), Dubreuil, 1883.--Nankeen yellow. Dwarf. Garden, standard, bedding. One of the best of its class. Prune 34 .

Persian Yellow (Austrian Briar), Willock, 1838. -Deep golden yellow. Vigorous. Garden, bush. Quite a distinct shade of yellow. Summer-flowering. Prune 19.

Petit Constant (poly pom.), Soupert et Notting, 1890.-Deep salmon pink, orange buds. Dwarf. Bedding, edging. Fragrant. Prune 34.

Pharisäer (H.T.), Hinner, 1901.-Rosy white, shaded pale salmon. Vigorous, upright growth. Exhibition, garden, standard, bedding, glass. An excellent Rose. Very fragrant. Prune 4 or 5.

pimpinellifolia (Species).--Native of Britain. Blush white. Moderately vigorous. Garden. Very early single Scotch Rose. Known as the "Burnet Rose." Summer-flowering. Prune 28 (21).

Pissardii (Species).-White. Semi-climber. Pillar. A perpetual-flowering pillar Rose. Fragrant. There is also a pink form of this variety, more vigorous but less perpetual. Prune 28 or 39 . flora."

polyantha grandiflora.-See " multiflora grandipolyantha simplex.-See " multiflora."

pomifera (Species).-Blush. Vigorous. Garden, bush. Large hips. Single-flowered. Summer-flowering. Prune 28. 
President Bouché (Pernet), Pernet-Ducher, 1917. -Coral red. Vigorous. Garden, bedding, glass Prune 4 or 42.

President Wilson (H.T.), Easlea, 1917.-Shrimp pink. Vigorous. Bedding. Prune 4.

Prima Donna (H.T.), W. Paul \& Son, 1917.Orange-yellow. Vigorous. Garden, bedding. Prune 4.

Prince Charming (H.T.), Hugh Dickson, 1916.Copper and old gold. Vigorous. Bedding. A beautiful variety. Semi-double. Prune 4.

Prince de Bulgarie (H.T.), Pernet-Ducher, 1902. -Pale tinted rose, shaded apricot. Vigorous. Garden, standard, bed'ding, glass, town. Variable in colour. A fine garden rose. Fragrant. Prune 4 or 42.

Princess Mary (H.T.), Elisha J. Hicks, 1915.Scarlet crimson. Moderate. Bedding, d'ecorative. Single-flowered. Prune 4.

Provence, Common (Prov.), 1596.-Rosy pink. Vigorous. Garden, bush. Known as the old "cabbage" rose. Summer-flowering. Very fragrant. Prune 11.

Provence, White.-See “White Provence."

Purity (H, wich.), Hoopes \& Thomas, 1917.-Pur. white. Very vigorous. Pillar, arch, pergola, weeping standard. Handsome foliage, flowers large and fragrant. Prune 39.

Queen Mab (C.). W. Paul \& Son, 1896.-Rosy apricot, shaded orange. Moderately vigorous. Garder. bedding. Prune 31 . 
Queen of Musk (Paul's) (Hybrid Musk), Paul and Son, 1912.-Pale pink. Vigorous. Garden, bedding. Prune 18.

R. arvensis (Ayr).-Pure white. Very vigorous. Arch. Prune 23.

Raymond (Pernet), Pernet-Ducher, 1917.-Peach, salmon centre. Vigorous. Bedding. Prune 20.

Red Cross (H.T.), A. Dickson \& Sons, 1916.Orange scarlet. Vigorous. Bedding. Prune 4.

Red Letter Day (H.T.), A. Dickson \& Sons, 1914. -Glowing scarlet crimson. Vigorous. Garden, standard, bedding, decorative. Prune 4.

Refulgence (S. Briar), W. Paul \& Son, 1908.Scarlet crimson. Vigorous. A good dark Hybrid sireet Briar. Hardy. Almost single. Prune 22.

Reine Olga de Wurtemberg (H.T.), Nabonnand, 1881.-Bright crimson. Vigorous climber. Pillar, arch, pergola, wall. Nearly evergreen. Prune 10 or 39.

Réné André (wich. ramb.), Barbier, 1900.-Dark saffron yellow, shaded orange red. Vigorous. Pillar, arch, pergola, weeping standard, wall, screen. Early summer-flowering. Fragrant. Prune 37 or 39.

Rêve d'Or (N.), Ducher, 1869._-Buff yellow. Vigorous climber. Arch, pergola, S. or W. wall. Flowers again freely in the autumn. Prune 32 (b) or 39.

Richmond (H.T.), The E. G. Hill Co., 1905.Bright light crimson. Vigorous. Garden, standard, 1)edding, glass. Very free flowering. A good buttonhole Rose. Very fragrant. Prune 2. 
Rödhätte (poly. pom.), Poulsen, 1912.-Rosy crimson. Drarf. Bedding. Fragrant. Prune 34.

Rosa Mundi (Gallica).--Red, striped white.Vigorous. Garden. The best of the striped Roses. The variety often grown as " York and Lancaster." Summer-flowering. Fragrant. Prune 14 (b).

Rubella (Species). - Rose. Yigorous. Bush. Very early summer-flowering. Single-flowered. Prune 2ri

rubrifolia (Species), Dr. Swainson, 1803.--Soft rose. Vigorous. Garden. Stems and foliage very distinct in colour. Crown for the effect of foliage only. Summer-flowering. Known also as "ferruginea." Prune 28.

rugosa alba (rug.).-White. Vigorous. Bush, hedge, dell, shrubbery, town. A white sport from "rugosa rubra." Single-flowered. Fine foliage. Prune 35.

Sanders' White (wich. ramb.), Sanders, 1915.Pure white. Arch, pergola, weeping standard. The best white rambler. Prune 37 .

Sarah Bernhardt (H.T.), Dubreuil, 1907.-Scarlet crimson, shaded. Vigorous. Garden, pillar, bush, hedge, pegging down. Semi-double. Prune 6 or 41 .

Scarlet Climber (Paul's) (Hybrid wichuraiana), IV. Paul \& Son, 1916.--Scarlet. Very vigorous. Pillar, arch, glass. Semi-double. Prune 20, 39, or 43.

Seagull (H. wich.), Pritchard, 1907.-.Vigorons. Pillar, arch. Fragrant. Prune 39.

sericea, pteracantha (Species), Vilmorin, 1905.White. Garden, bush. Noteworthy for its transhucent and huge red thorns on the young wood. Very early summer-flowering. Prune 28. 
sericea (Species).-White. Vigorous. Garden, bush. Has only four petals. Prune 28.

Severine (Pernet), Pernet-Ducher, 1919.-Vivid orange. Vigorous. Garden, bediding. Prune 20.

Shower of Gold (wich. ramb.), Paul \& Son, 1910. -Golden yellow, changing to pale yellow. Vigorous climber. Pillar, arch, pergola. Early mid-season. Effective foliage. Prune 37 or 39.

sinica Anemone (Hybrid of Species), F. Schmitt, 1895. - Silvery-pink, shaded rose. Vigorous. Semiclimber. Screen, 'S. or W. wall. A charming singleflowered rose. Early summer-flowering. Prune 29.

Source d'Or (wich ramb.), Turbat \& Co., 1912.Golden yellow. Very vigorous. Arch, pergola. Fine colour. Prune 37

Stanwell Perpetual (Perpetual Scotch) Lee.-Pale blush. Vigorous. Garden, standard, hedge. Flowers very early, and is also good in autumn. Very fragrant. Prune 36.

Sunburst (H.T.), Pernet-Ducher, 1911.-Orange or cadmium yellow to creamy white. Vigorons. Glass. Very variable in colour. Prune 4 or 42.

Sunburst, Climbing (H.T.), Howard Rose Co., 1915.--Very vigorous. Pillar, S. or W. wall, close fence. A climbing sport from "Sunburst." Prune 10.

Sweet Briar, Common (S. Briar).-Pale pink. Vigorous. Bush, hedge. Fragrant foliage. Early stummer-flowering. Prune 22 (a).

Tausendschön (mult. ramb.), Schmidt, 1907.Rose-pink. Semi-climber. Pillar, pergola. Semidouble. Summer-flowering. Prune 27 or 39. 
Tea Rambler (mult. ramb.), Paul \& Son, 1903.Coppery pink. Very vigorous. Pillar, arch, pergola, bedge. Summer flowering. Prune 27 or 39.

T. F. Crozier (H.T.), Hugh Dickson, 1918.Canary yellow. Vigorous. Exhibition. Reliable. Prune 3.

The Garland (Hybrid Musk), Wells.-Blush, changing to white. Vigorous climber. Pillar, arch, pergola. One of the best summer-flowering climbers. summer-flowering. Prune 18 or 39.

The Queen Alexandra Rose (Pernet), S. McGredy and Son, 1917.-Vermilion, reverse of petals old gold. - Vigorous. Bedding. Unique colours; best in autumn. Very fragrant. Prune 20.

Thoresbyana.-See "Bennett's Seedling."

Toreador (H.T.), W. Paul \& Son, 1919.-Crimson and yellow. Vigorous. Bedding. Semi-double. Prune 20.

Trier (Hybrid Musk), P. Lambert, 1904.-Creamy white, edged fawn.-Semi-climber. Bush, pillar. Flowers again in the autumn. Prune 18 or 39.

Triomphe d'Orleanaise (poly. pom.), J. Peanger, 1912.-Brilliant carmine. Vigorous. Garden, bedding. Prune 34.

Ulrich Brunner (H.P.), Levet, 1881.-Cherry red. Very vigorous. Exhibition, garden, town. A good all-round Hybrid Perpetual. Very fragrant. Prune 6 or 7 .

Una (H. Briar), Paul \& Son, 1900.--Pale cream. Vigorous semi-climber. Bush, pillar, arch, hedge. Semi-single. Summer-flowering. Prune 29 or 39.

Victor Hugo (H.P.), Schwartz, 1884.-Dazzling crimson, shaded. Moderate. Exhibition, garden. Grand colour. Fragrant. Prune 2 or 3. 
Viscountess Folkestone (H.T.), Bennett, 1886.Cream.y-white, shaded flesh. Vigorous. Garden, standard, bedding, glass. One of the best bedding Roses. Very fragrant. Prune 4 or 42.

W. C. Clark (H.T.), W. Paul \& Son, 1917.-Deep maroon crimson. Very vigorous. Garden, bedding. Very dark. Prune 6.

W. C. Gaunt (H.T.), A. Dickson \& Sons, 1916.Scarlet crimson Vigorous. Garden, bedding. A most useful variety. Prune 4.

White Manan Cochet (T.), Cook, 1897.-White, tinged lemon. Vigorons. Exhibition, garden, standard, glass. An especially good Tea. Prune 6, 7, or 42 .

White Provence (Prov.).-Grimwood, 1777.Paper white. Vigorous. Garden, bush. Summerflowering. Prune 11.

White Rambler (Pemberton's) (Hybrid multiflora), Rev. J. H. Pemberton, 1914.-Pure white. Very vigorous. Pillar, arch. Late flowering. Prune 27 or 39 .

wichuraiana (wich. ramb.), Species from Japan, 1880.-White, with yellow anthers. Very vigorous. Creeping. Late summer-flowering. Single-flowered. Fragrant. This was first discovered by Dr. Wichura in 1860, and introduced into Europe about 1873. Prune 37.

William Allen Richardson (N.), Veuve Ducher, 1878.-Deep orange yellow, white edge. Vigorous climber. Garden, standard, bush, pillar, pergola, pegging down, $\mathrm{S}$. or $\mathrm{W}$. wall. Unique in colour but variable. Good under glass. A good buttonhole Rose. Prune 32 (a), 39, 41, or 42 . 
William Shean (H.T.), A. Dickson \& Sons, 1906. -Creanly pink. Vigorous. Exhibition, glass. Prune 5.

W. R. Smith (T.), Henderson, 1908.-White, tinged blush. Vigorous. Exhibition, garden, standard. Prune 4 or 5 .

xanthina (Species).-Vigorous. Garden, hedge. Lemon yellow. Single-flowered. Early summerflowering. Known also as " Ecr." Prune 28.

York and Lancaster (D.).-Pale rose or white, sometimes striped. Vigorous. Garden. Summerflowering. Fragrant. "Rosa Mundi" is generally, but incorrectly, known under this name. Prune $\overline{14}$.

Yvonne Rabier (poly. pom.), Turbat \& Co., 1910. -White. Garden, bedding, bush. Very free and perpetual flowering. Prune 34.

Yvonne Vacherot (H.T.), Soupert et Notting, 1906.-Porcelain white, tinted blush. Moderately vigorous. Exhibition. Prune 5.

Zéphirine Drouhin (H.B.), Bizot, 1873.-Bright carmine pink. Vary vigorous. Garden, pillar, hedge, $S$. or W. wall, town. Thornless. Very fragrant. Prune 10 or 39 .

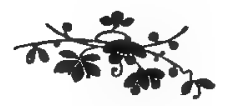




\section{A Descriptive List of some of the most promising Newer Roses.}

Archie Gray (H.T.), Hugh Dickson, 1920.-Intense scarlet. Vigorous. Exhibition, garden. Prune 3.

Captain Georges Dessirier (H.T.), Pernet-Ducher, 1920.-Orange yellow. Vigorous. Bedding. Prune 4.

Coral Cluster (poly. pom.), R. Murrell, 1920.-Pale coral pink. Vigorous. Bedding, glass. Unusually large truss. Very beautiful. Prune 34.

Florinda Norman Thompson (H.T.), A. Dickson \& Sons, 1920.-Rose pink, shaded lemon. Vigorous. Exhibition. Prune 3.

Francklin (Pernet), Pernet-Ducher, 1920.-Rosy flesh. Vigorous. Garden, bedding. Prune 20.

Glowworm (Pernet), W. Easlea, 1920.-Orange scarlet. Vigorous. Bedding, decorative. Singleflowered. Prune 20.

Kosters Orleans (poly. poll.), Koster, 1920.scarlet. Vigorous. A deeper coloured "Orleans." Very fine. Prune 34.

Lady Maureen Stewart (H.T.), A. Dickson \& Sons, 1920,-Dark crimson. Vigorous. Bedding. Fragrant. Prune 4.

Lulu (H.T.), W. Easlea, 1920.-_Orange salmon. Vigorous. Bedding. Semi-double. Prune 4. 
Manifesto (H.T.), S. McGredy \& Son, 1920.Flesh pink, shaded salmon. Vigorous. Exhibition, garden, bedding. Prune 4.

Mrs. Arthur Johnson (H.T'), S. McGredy \& Son, 1920.-Bright yellow. Vigorous. Bedding. Very fine. Prune 4.

Mrs. Charles Lamplough (H.T.), S. McGredy \& Son, 1920.-Lemon chrome. Very vigorous. Exhibition, garden. A fine Rose. Prune 3.

Mrs. Curnock Sawday (H.T.), Elisha J. Hicks, 1920.--Rosy white. Vigorous. Bedding. Prune 4.

Mrs. H. R. Darlington (H.T.), \&. McGredy \& Son, 1920.-Lemon white. Vigorous. Exhibition. Prine 3.

Mrs. W. Lindsay (H.T.), Hugh Dickson, 1920.Fawn yellow. Vigornis. Bedding. Prine 4.

Victory (H.T.), S. McGredy \& Son, 1920.-Intense scarlet. Vigorous. Exhihition, standard, bedding. Prune 3.

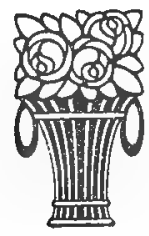




\section{Roses Mentioned in Descriptive List.}

Arranged accorting to the classes to which they belong.

\section{Alba.}

Celestial

Maiden's Blush.

Austrian Briar (R. lutea).

Austrian Copper

Harrisonii

Yellow

Persian Yellow

Ayrshire (R. arvensis).

Bennett's Soedling (Thoresbynna) Rআa arrenkis

Dundee Rambler

Banksia (R. Banksia).

White

Yellow

China (R. indica).

Charlotte Klemm

Common China (Old Monthly)

Comtesse du Cayla

Cramoisie Supérienre (t'limbinn)

Fabvier
Fellenberg

Laurette Messimy

Mme. E. Resal

old Crimson

Queen Mab

\section{Damask ( R. Damascena).}

Commandant Beaurepaire

Lady Curzon
Mrs. O. G. Orpen

York and Lancaster 


\title{
Gallica (R. Gallica). \\ Rosa Mundi
}

Hybrid Bourbon ( $R$. indica Bourboniana).

Zéphirine Drouhin

\section{Hybrid Brockata.}

Mermaid

\begin{abstract}
Hybrid Briar (R. canina).
Una
\end{abstract}

Clytemnestra

Danäe

Mme. D'Arblay

Moonlight
Pax

Queen of Musk (Paul's)

Trier

The Garland

\section{Hybrid multiflora.}

White Rambler (Pemberton's)

\section{Hybrid Noisette.}

Lemon Pillar (Paul's)

Mme. Alfred Carrière

\section{Hybrid Perpetual.}

Ards Rover

Candeur Lyonnaise

Captain Hayward

Commandant Félix Faure

Coronation

Frau Karl Druschki

Général Jacqueminot
Gloire de Chédane-Guinoisseau Horace Vernet Hugh Dickson Louise Crette Mrs. John Laing Ulrich Brunner

Victor Hugo 


\section{Hybrid Tea.}

Admiral Ward

Alesander Emslie

Antoine Rivoire

Archio Gray

Augustus Hartmann

Augustine Guinoisseau

Avoca

Benedicte Sequin

Bessie Brown

Betty

Captain Georges Dessirier

Caroline Testout

Charles E. Shea

Charles K. Douglas

Château de Clos Vougeot

Cheerful

Cherry Page

Chrissie Mackellar

Clarice Goodacre

C'limbing Caroline Testout

., Mrme. Abel Chatenay

, Lady Ashtown

, Mélanie Soupert

" Mrs. W. J. Grant

, Papa Gontier

, Paul Lédé

, Sunburst

Colonel ()wwalk Fitzgerald

Countess of Warwick

Countess of Shafteshury

Covent Garden

Cupid

C. V. Haworth

Cynthia Forde

- Dean Hole

Donald McDonald

Doithy Page-Roheris

Dowager Countess of Rorlen
Dr. Joseph Drew

Duchess of Wellington

. , "Westminster

Ecarlate

Edel

Edgar M. Burnett

Edith "Cavell

E. Godfrey Brown

Elegante

Emma Wright

Flame of Fire

Florence Forrester

$$
\begin{array}{ll}
\text { " } & \text { H. Veitch } \\
\text { " } & \text { Pemberton }
\end{array}
$$

Florinda Norman Thompson

Frances Gaunt

François Crousse

G. C. Waud

General McArthur

George Dickson

Gladys “Holland

Golden Ophelia

, Spray

Gorgeous

Gruss an Teplitz

Gustav Grünerwald

Gustare Régis

Jadley

II. D. M. Barton

Helen Chamberlain

Henrietta

U. V. Machin

II. P. Pinkerton

Hoosier Beanty

Irish Afterglow

. Irish Elegance

, Tireflame

, Fireflame (climbing) 
Hybrid Tea-continued.

J. B. Clark

Joanna Bridge

Jonkheer J. L. Mock

Joseph Hill

$\mathrm{K}$. of $\mathrm{K}$.

Kootenay

Lady Alice Stanley

, Ashtown

" Greenall

, Maureen Stewart

"Pirrio

,Waterlow

La France

"Tosca

Lamia

Laurent Carle

Liberty

Lieutenant Chaur6

Lilian Moore

Los Angeles

Louise Baldwin

-Lulu

Mabel Drew

Mme. Abel Chatenay

C. Martel

", Jules Bouch6

, Jules Grolez

"Léon Pain

, Ravary

, Segond-Weber

Manifesto

Marcella

Marchioness of Ormonde

Margaret

Margaret Dickson Hamill

Mélanie Soupert

Melody

Mildred Grant

Miriam

If iss Willmott
Modesty

Molly Bligh

Mrs. Aaron Ward

"A. E. Coxhead

" Alfred Tato

" Arthur Johnson

, Bertram J. Walker

" Bryce Allan

" Charles Lamplough

" Charles Russell

" Curnock Sawday

" C. V. Haworth

" Dunbar Buller

"Dunlop Best

, E. G. Hill

, Elisha J. Hicks

, E. Powell

, Franklin Dennison

" George Norwood

, " Marriott

" "Shawyer

, Henry Morse

, H. D. Greene

, H. R. Darlington

, J. H. Weleh

, Mackellar

" Mona Hunting

, R. D. McClure

, Redford

, Rosalie Wrinch

, Theodore Roosevelt

, Wakefield Christie-Miller

" W. Lindsay

Nellie Parker

Noblesse

old Gold

Ophelia

Pharisäer

President Wilson

Prima Donina 
Hgbrid Tea-continued.

Prince Charming

Prince de Bulgarie

Princess Mary

Red Cross

Red Letter Day

Reine Olga de Wurtemberg

Richmond

Sarah Bernhardt

Sunburst

Emily Gray
Purity

Emily Gray
Purity

\author{
T. F. Crozier \\ Toreador \\ Victory \\ Viscountess Folkestone \\ W. C. Clark \\ W. C. Gaunt \\ William Shean \\ Yronne Vacherot
}
Hybrid wichuraiana.
Scarlet Climber (Paul's)
Seagull

Moss (R. Centifolia muscosa).

Blanche Moreau

Common Moss

Crested Moss

Perpotual White

multiflora rambler (R. multiflora).

Aglaia

Blush Rambler

Carmine Pillar

Crimson Rambler

Goldfinch

Tausendschön

Tea Rambler

Noisețte (R. moschata Noisettiana).

Aimeé Vibert (climbing)

Alister Stella Gray

Fortune's Yellow
Maréchal Niel

Rêve d'Or

William Allen Richardson

\section{Pernetiana (R. 1 utea).}

Arthur R. Goodwin

May Marriott

Christine

Constance

Francklin

Glowworm

Golden Emblem

Independence Day

Isobel

Louise Catherine Breslau

Lyon Rose

Mme. Edouard Herriot

Mrs. Ambrose Ricardo

" Farmer

"Wemyss Quin

President Bouché

Raymond

Severine

The Queen Alexandra Rose 
Perpetual Scotch (R. spinosissima).

Stanwell Perpetual

\section{Polyantha (pompon) (R. multiflora).}

Ennchen Müller

Aschenbrödel

Canarienvogel

Cecile Brunner

Coral Cluster

Ellen Poulsen

Eugénie Lamesch

George Elgar

Jessie

Katharine Zeimet

Koster's Orleans

Léonie Lamesch

Little Meg
Maman Turbat

Marie Pavio

Mme. Jules Gouchault

Mrs. W. H. Cutbush

Mrs. W. H. Cutbush (climbing)

Orleans Rose

Orleans Rose (climbing)

Perle d'Or

Petit Constant

Rödhätte

Triomphe d'Orleanaise

Yvonne Rabier

Common Provence

Provence (R. centifolia).

White Provence

rugosa (R. rugosa).

Blanc double de Coubert

Conrad F. Meyer

Nova Zembla

rugosa alba

, rubra

Sempervirens (R. sempervirens).

Félicité-et-Perpétue

Species and Hybrid of Species.

alpina

, pyrenaica

altaica

Andersoni

Hebe's Lip

Hugonis

lucida.

s plena

macrantha moschata

$$
\begin{array}{ll}
" & \text { alba } \\
, & \text { (fl. pl.) } \\
" & \text { himalayica }
\end{array}
$$

Moyesii

pimpinellifolia

pissardii

polyantha grandiflora

, simplex 
Species and Hybrid of Species-cuntinued.

pomifera

Rubella

rubrifolia

sericea sericea pteracantha sinica Anemone xanthina

Sweet Briar and its Hybrids (R. rubiginosa).

Amy Robsart

Anne of Geierstein

Flora McIvor

Janet's Pride

Jeanie Deans

Julia Mannering
Lady Penzance

Lord

Meg Merrilies

Refulgence

Sweet Briar (Common)

Tea (R. indica odorata).

A. Hill Gray

Anna Olivier

Bouquet d'Or

Climbing Niphetos

Corallina

Glaire de Dijon

G. Nabonnand

Lady Hillingdon

, Hillingdon (climbing)

, Plymouth

, Roberts

Mme. Antoine Mari
Mme. Constant Soupert

" jean Dupuy

, Jules Gravereaux

Maman Cochet

Marie van Houtte

Medea

Molly Sharman-Crawford

Mrs. Campbell Hall

„ Foley Hobbs

, Herbert Stevens

White Maman Cochet

W. R. Smith

wichuraiana Ramblers ( $R$. wichuraiana).

Albéric Barbier
American Pillar
Aviateur Bleriot
Cliatillon Rambler
Christine Wright

Coronation

Débutante 
wichuraiana Ramblers-continued.

Diabolo

Dorothy Perkins

Dr. Van Fleet

Elisa Robichon

Ethel

Evangeline

Excelsa

François Guillot

, Juranville

Gardenia

Gerbe Rose

Hiawatha

Jean Guichard

Jersey Beauty
Joseph Billard
", Lamy
Lady Gay
' Godiva
Léntine Gervais
Minnehaha
Réné André
Sanders' White
Shower of Gold
Source d'Or
wichuraiana

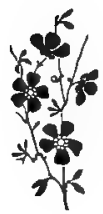



INSTRUCTIONS

FOR

PRUNING ROSES. 
Plate I.

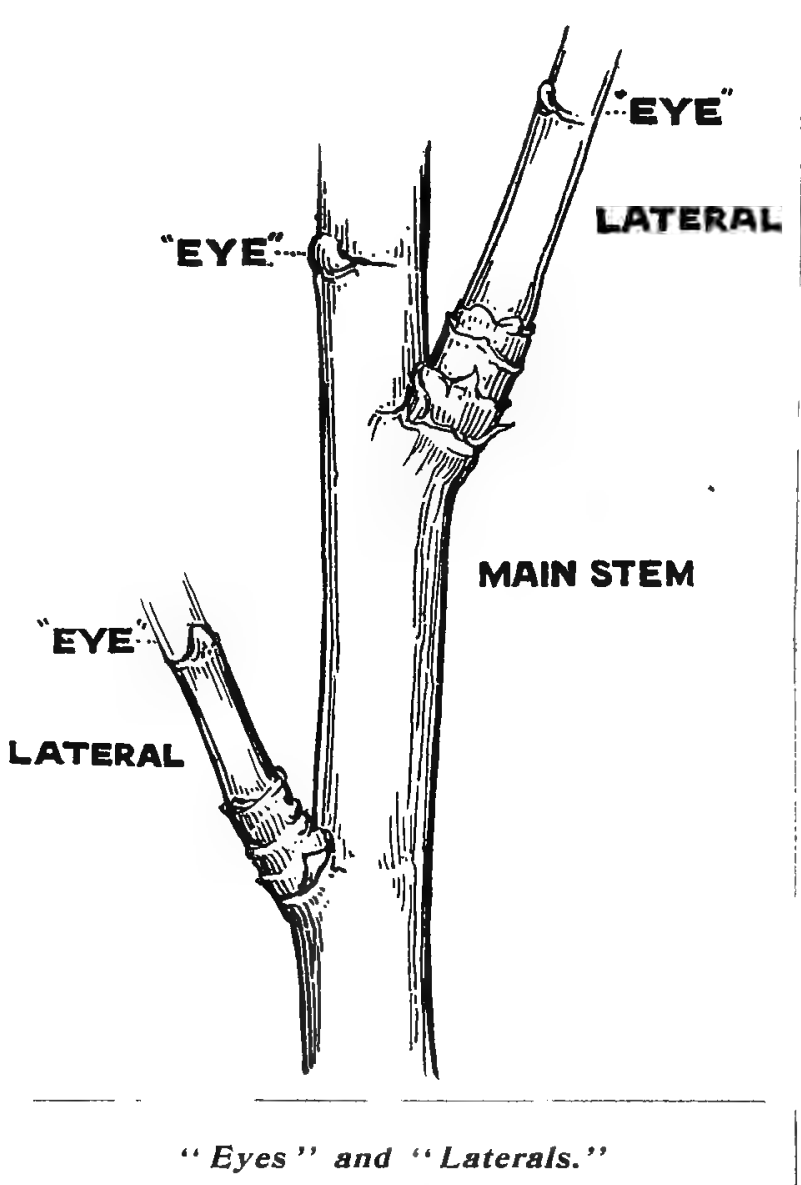

These terms are frequently used throughout these instructions and may require explanation. As will be seen by the above Illustration, by an "Eye" is meant a bud on the main stem or any of the other shoots. By a "Lateral" is meant a side shoot on any leading branch or shoot. 


\title{
INTRODUCTORY REMARKS.
}

\author{
THE following general directions and explanations \\ should be carefully read before proceeding to carry \\ out the instructions afterwards given, or the reader \\ may fail to grasp the meaning they are intended to \\ convey.
}

The late Rev. A. Foster-Melliar in his "Book of the Rose" pointed out that the necessity for pruning arises in a great measure from the natural growth of the Rose. "By watching," he said, "an unpruned Rose-tree, either wild or cultivated, it will be found that the first strong shoot flowers well the second season, but gets weaker at the extremity in a year or two, and another strong shoot starts considerably lower down, or even from the very base of the plant, and thus soon absorbs the majority of the sap, and will eventually starve the original shoot and be itself thus starved in succession by another. A Rose in a natural state has thus every year some branches which are becoming weakened by the fresh young shoots growing out below them. This is one of the principal reasons why pruning is necessary. A Rose is not a tree to grow onwards and upwards, but a plant which in the natural course every year or two forms fresh channels for the major portion of the sap, and thus causes the branches and twigs abnve the new shoots to diminish in vitality." 
From this it may be gathered that Nature's method of getting rid of the undesirable parts of the plant is the slow one of gradual starvation; and it is wasteful, in that during the process even the strong shoots are likely to become weakened, injured or diseased.

The Rosarian's aim is to have healthy, well-formed plants; and from them to obtain either large perfect blooms or many beautiful ones. To do this he must prune. The art of pruning is the scientific cutting away of those parts of the plant which the study of Nature has shown to be useless for producing the best results, and thus to throw the whole strength of the plant into the most satisfactory shoots.

Pruning consists of two distinct operations: (I) Thinning out, which is the complete removal of all dead, weak, misplaced, unripe, or otherwise useless shoots ; (2) The shortening of those shoots which are left, to such an extent as to give the best chance of obtaining what is desired.

The most frequent errors made in pruning are (I) leaving too many shoots when thinning out; (2) pruning severely the shoots of varieties which require little, if any, shortening ; (3) pruning lightly the varieties which require severe pruning; (4) leaving Roseplants crowded with shoots and cutting these to a uniform length all over the plant in the way that a hedge is clipped.

In thinning out a shoot it should be either cut clean away to the base of the plant, or to its starting point on the older shoot from which it springs, as the case may be. When the plant has been pruned the shoots should be left as nearly as possible equi-distant 
from each other, and regularly arranged round it so that it presents a well-balanced appearance on all sides. When pruning a shoot it should always be cut to a dormant leaf bud, or "eye" (Plate 2), as it is called, pointing outwards. In order to keep the plants in a healthy and vigorous condition some of the shoots which are more than two years old should each year be removed to make room for younger and more vigorous growths.

Roses require to be somewhat differently pruned according to the purpose for which they are grown. For instance, shoots must be cut back severely if the plant be required for the production of exhibition Roses, but if for ordinary garden decoration the shoots must be left longer; if to form bushes they must be left still longer, and if to clothe pillars, arches, \&c.,. some of the shoots will scarcely require shortening at all.

To cut down Rose-plants as is advised in the following pages in order to obtain exhibition blooms may seem to the beginner likely to endanger the life of the plants, whereas in practice the harder the shoots are cut in the Spring the stronger will be the new growths made during the Summer.

When pruning a Rose for the decoration of the garden it should be borne in mind that it is a decorative garden plant, whether it be the large bush or pillar to stand alone, or the dwarfer kind to be massed with others in a bed. As a large range of kinds is available for decorative purposes it is necessary in pruning, as will be seen by the instructions which follow, to take into account their individual habits and. requirements. 
Plate II.

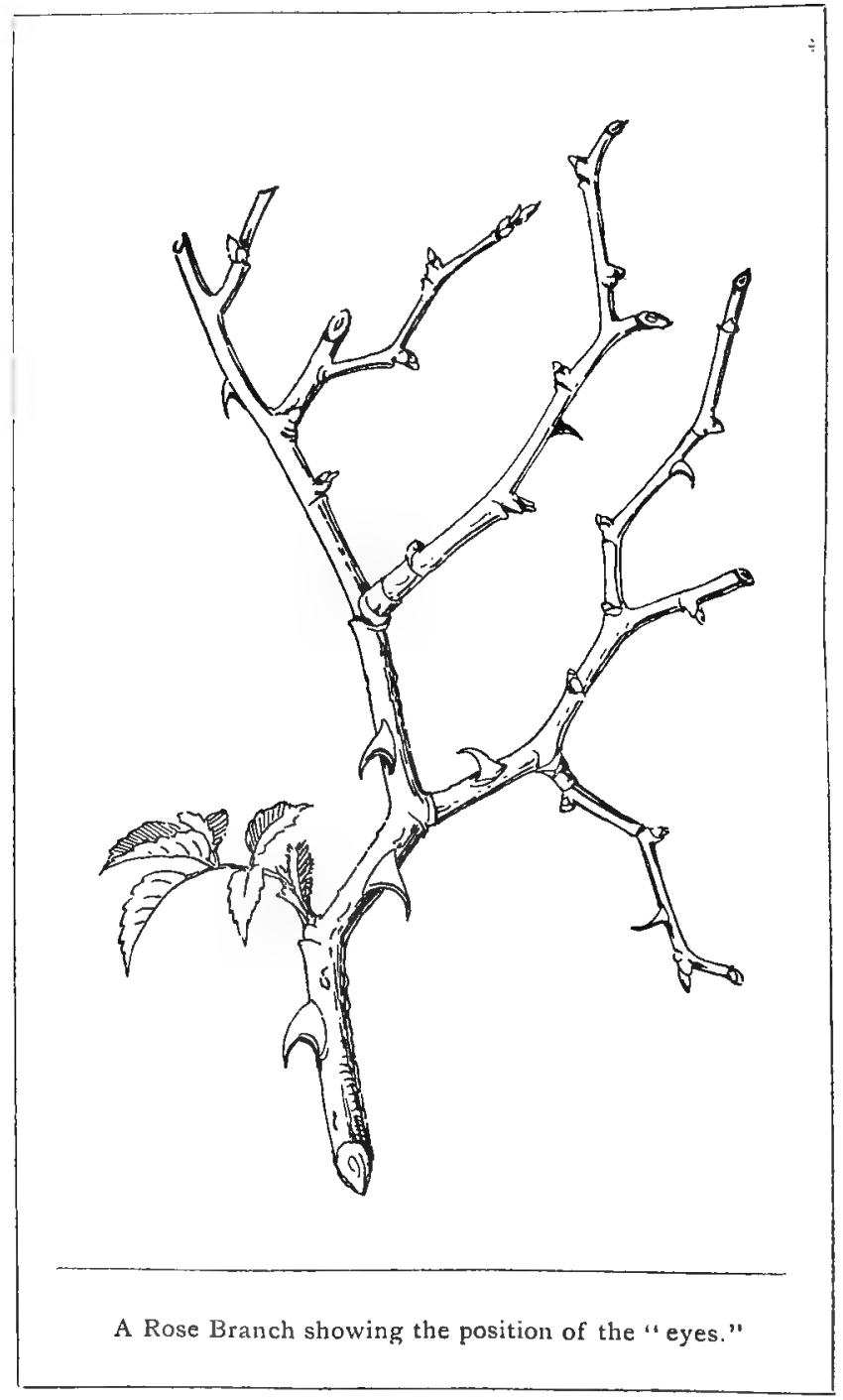





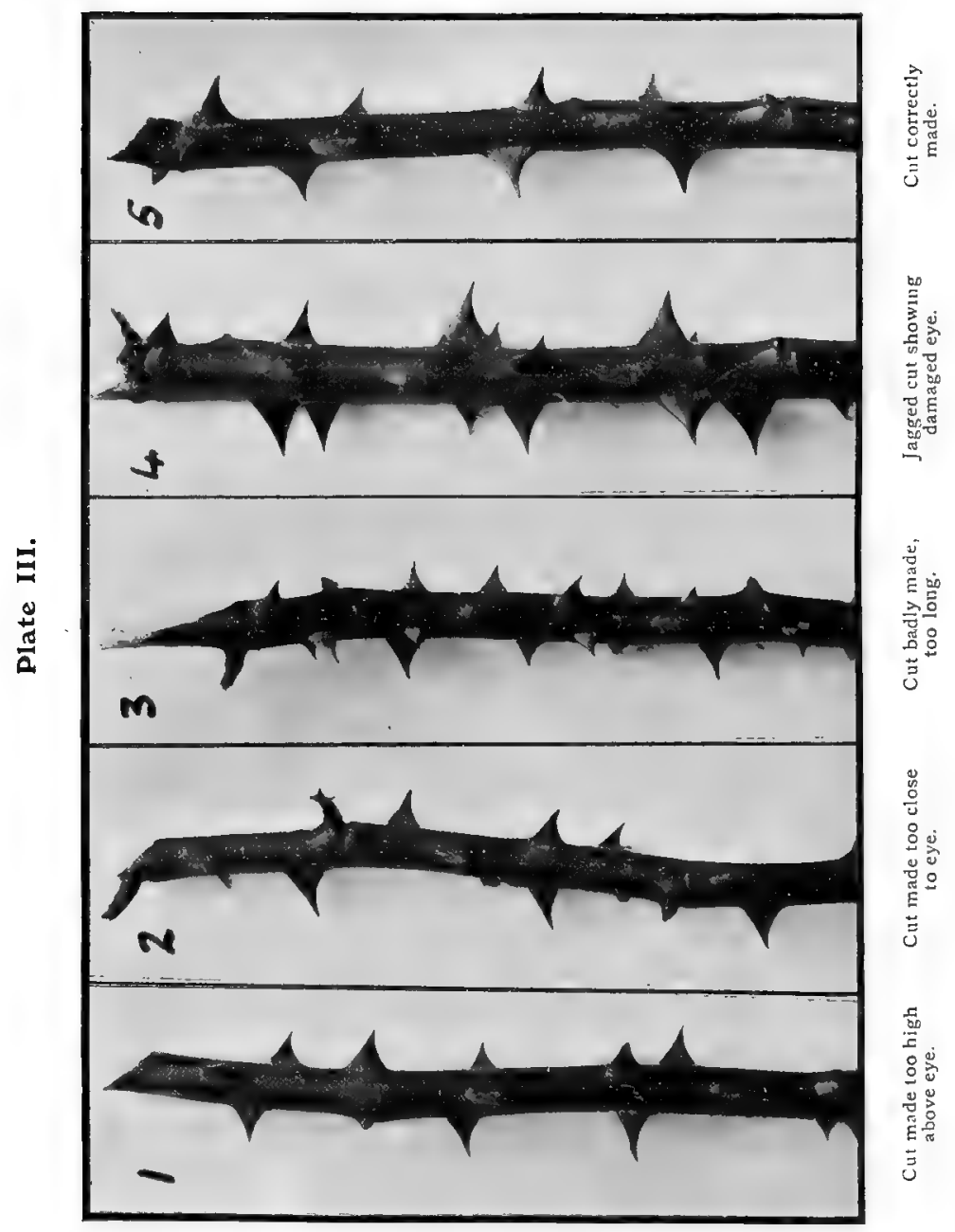


A large and strong-growing Rose usually requires some two or three years after planting to reach its full size, and it is therefore during those first years that the plant has to be built up, and its framework, as it were, formed

\section{INSTRUMENTS FOR PRUNING.}

These need be but few in number, viz., a pruning knife of moderate size, a hone on which to sharpen it, a kneeling pad, a small saw, and an easy pair of garden. ing gloves. It is very important that the knife be kept with a keen edge. This is best done by first obtaining a small piece of old linoleum and spreading upon it some Wellington knife powder. An occasional rub or two of the pruning knife over the surface will produce a much keener cutting edge than that produced by rubbing on an ordinary oil stone. Secateurs may be used for the removal of the hard old wood, but they must be used with great care for general pruning, or a bruising of the cut shoot will result. Only those secateurs should be used which are sharp and have both blades accurately adjusted.

\section{HOW TO USE THE INSTRUCTIONS.}

Refer to the Alphabetical Descriptive List of Roses for the name of the variety to be pruned. At the end of its description will be found a reference, or references to the method, or methods of pruning recommended. Thus: Prune 4 and 5, means prune according to Instructions 4 and 5 .

Occasionally a short list of varieties is introduced after an "Instruction" as typical of the varieties requiring the treatment indicated, but not as a complete list. 


\section{THE TIME OF PRUNING}

\section{HYBRID PERPEUALS, HYBRID TEAS, AND} TEAS AND NOISETTES.

Dwarf and Standard Hybrid Perpetuals, Hybrid Teas and Pernetianas

Should be pruned during the month of March.

Duarf and Standard Teas and Noisettes

Should be pruned during the month of April.

Climbing Yarieties of Hybrid Perpetuals, Hybrid Teas, and Teas and Noisettes

Should be thinned out after flowering in the Summer and pruned in March.

For the time of pruning other classes of Roses see Instructions I I -37 . 



\title{
HOW TO PRUNE THE DIFFERENT KINDS OF ROSES.
}

\author{
INSTRUCTION I.
}

HOY TO PRUNE THE FIRST TIME AFTER PLANTING.

All Roses the first time after planting should be pruned severely, that is to say they should be cut down to within 5 or 6 inches of the ground. Even in the case of strong growing climbing varieties only the strongest shoot should be left more than $\mathrm{r}$-ft. in length. (See Plate IV.)

Roses planted in the Autumn should be pruned in the following Spring, and if planted in the Spring should be pruned at the time of planting.

After the first year they should be pruned in accordance with the Instructions which follow. 


\section{INSTRUCTION 2.}

\section{HYBRID PERPETUALS, HYBRID TEAS, AND TEAS AND NOISETTES.}

\section{Yarieties which require hard pruning.}

For time of pruning see page 88.

For Garden Purposes.-Where the plants are grown chiefly for the ornamentation of the garden and high quality of individual blooms is not required, the method of pruning to be employed will be as follows :All the dead, weak and unripe shoots must be cut clean away to the base whence they started, and the centre of the plant thinned out sufficiently to allow room for the new growth which is to come after pruning. This done only the stronger and well-ripened shoots of the previous year's growth will be left, and these should be cut back to within four to six "eyes" from the base from whence the shoot started. In pruning Roses tor garden purposes greater attention should be given to the symmetrical formation of the plant, and more shoots (according to the vigour of the variety) from the base should be allowed to remain than when pruning for exhibition purposes. 



\section{INSTRUCTION 3 .}

\section{HYBRID PERPETUALS, HYBRID TEAS, AND TEAS AND NOISETTES.}

\section{Yarieties which require hard pruning.}

For time of pruning see page 88.

For Exhibition Purposes.-Roses which are weak growers require to be cut hard back at the time of pruning, by which it is intended to convey that all the dead, weak and unripe shoots must be cut clean away to the base whence they started, and the centre of the plant thinned out sufficiently to allow room for the new growth which is to come after pruning. This done only the stronger and well-ripened shoots of the previous year's growth will be left, and these should be cut back to within three or four, or less, "eyes" from the base whence the shoot started. It may be necessary, probably about the third week in May, as soon as the flower buds can be seen, to remove with the fingers (or carefully with a knife) some of the surplus shoots, especially those which are blind (that is without flower buds at the termination of the shoot), leaving only those which are most promising and well placed. 


\section{INSTRUCTION .4•}

\section{HYBRID PERPETUALS, HYBRID TEAS, AND TEAS AND NOISETTES.}

\section{Varieties which require moderate pruning.}

For time of pruning see page 88 .

For Garden Purposes.-Where the plants are grown chiefly for the ornamentation of the garden and high quality of individual blooms is not required, the method of pruning to be employed will be as follows:-Cut away to the base all dead, weak and unripe shoots. All shoots which have a tendency to cross one another must be cut out so that the plant may not become too crowded, a point which has special importance in dealing with the centre of the plant. The pruner will now have only the stronger and wellripened shoots of the previous year's growth to deal with, and these should be cut back so as to leave from six to eight "eyes" on each shoot. In pruning Roses for garden purposes greater attention should be given to the symmetrical formation of the plant, and more shoots (according to the vigour of the variety) from the base should be allowed to remain than when pruning for exhibition purposes. 


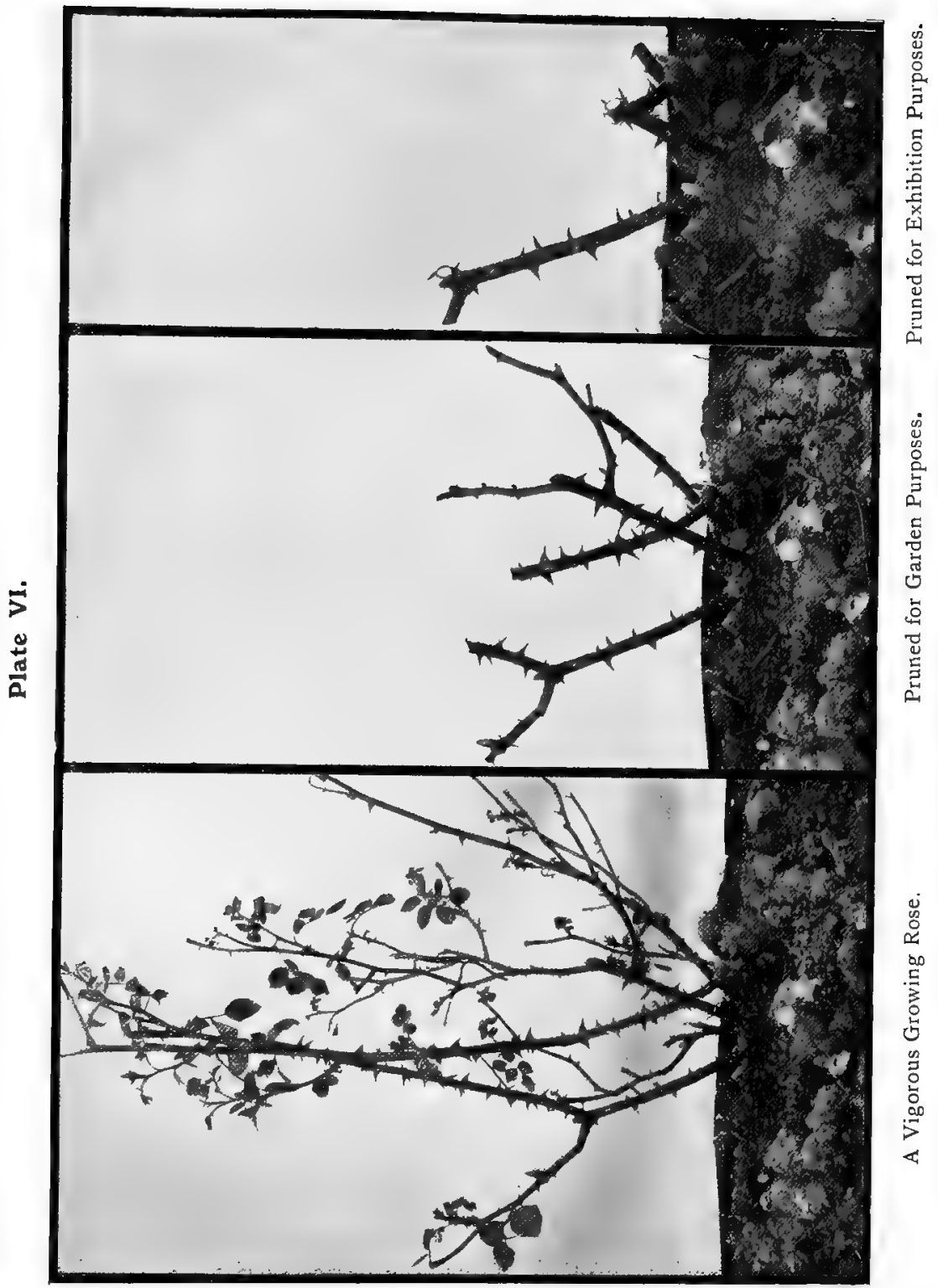



INSTRUCTION 5 .

\section{HYBRID PERPETUALS, HYBRID TEAS, AND TEAS AND NOISETTES.}

\section{Yarieties which require moderate pruning.}

For time of pruning see page 88.

For Exhibition Purposes. - These Roses need less severe pruning than those in Instruction 3. Cut away to the base all dead, weak and unripe shoots. All shoots which have a tendency to cross one another must be cut out so that the plant may not become too crowded, a point which has special importance in dealing with the centre of the plant. The pruner will now have only the stronger and wellripened shoots of the previous year's growth to deal with, and these should be cut back so as to leave from four to five "eyes" on each shoot. It may be necessary, probably about the third week in May, as soon as the flower buds can be seen, to remove with the fingers (or carefully with a knife) some of the surplus shoots, especially those which are blind (that is without flower buds at the termination of the shoot), leaving only those which are most promising and well placed. 
INSTRUCTION 6.

\section{HYBRID PERPETUALS, HYBRID TEAS, AND TEAS AND NOISETTES.}

\section{Yarieties which require light pruning.}

For time of pruning see page 88.

For Garden Purposes.-Where the plants are grown chiefly for the ornamentation of the garden and high quality of individual blooms is not required, the method of pruning to be employed will be as follows: All dead, weak and unripe shoots must be cut away to the base. Shoots which have a tendency to cross one another must be cut out, so that the plant, especially the centre, may not become too crowded. The longer and stronger shoots coming from the base should be reduced in length to about twelve inches; while from four to five "eyes" may be left on the laterals on the remaining older shoots. In pruning Roses for garden purposes greater attention should be given to the symmetrical formation of the plant, and more shoots (according to the vigour of the variety) from the base should be allowed to remain than when pruning for exhibition purposes. 


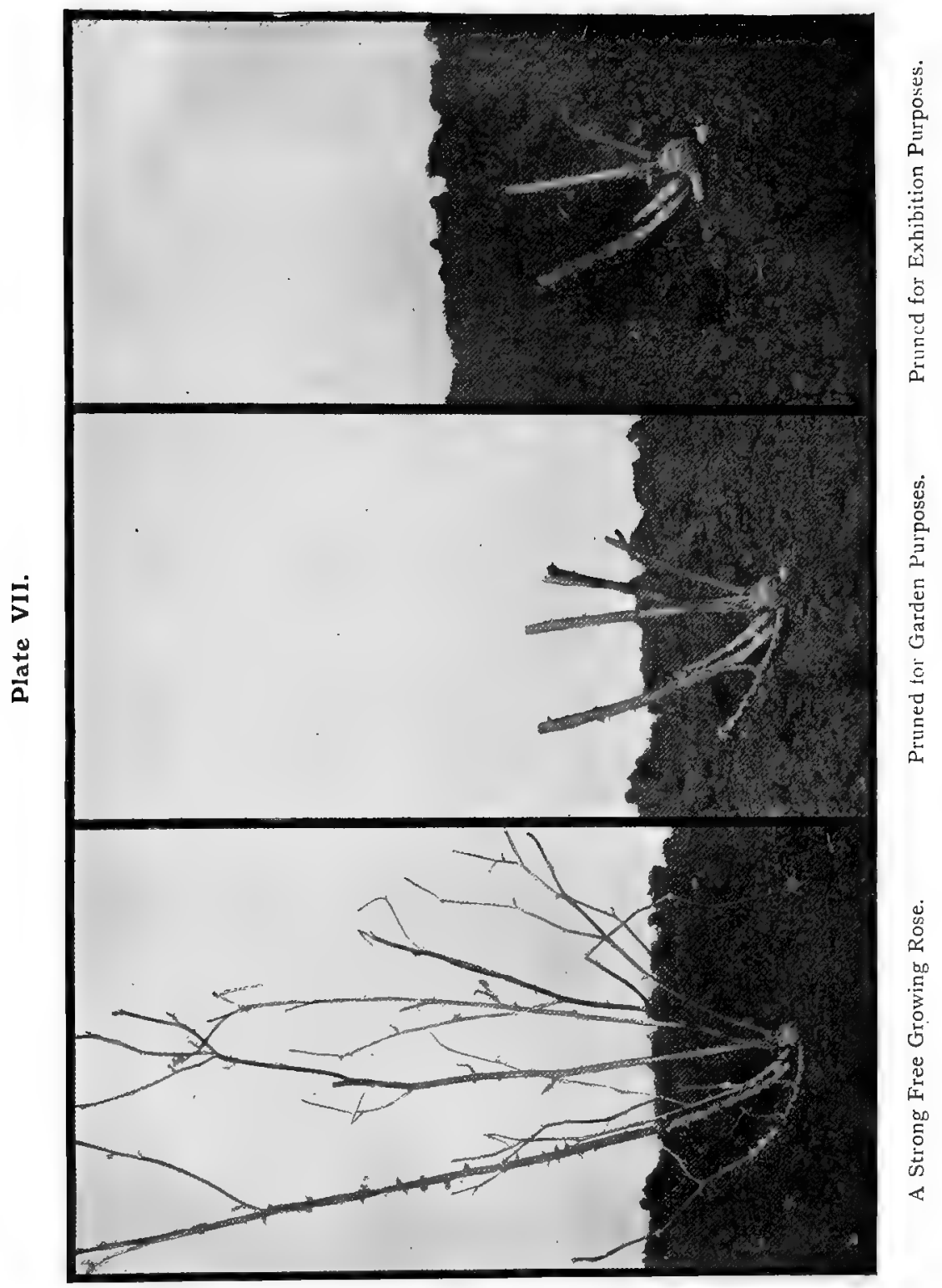





\section{INSTRUCTION 7 .}

\section{HYBRID PERPETUALS, HYBRID TEAS, AND TEAS AND NOISETTES.}

\section{Yarieties which require light pruning.}

For time of pruning see page 88.

For Exhibition Purposes.-In dealing with a class of Roses which are still stronger and freer growing than those referred to in the previous Instructions, a still less severe treatment is necessary. All dead, weak and unripe shoots must be cut away to the base. Shoots which have a tendency to cross one another must be cut out, so that the plant, especially the centre, may not become too crowded. The longer and stronger shoots coming from the base should be reduced in length to about eight inches; while from two to three "eyes" may be left on the laterals on the remaining older shoots. It will usually be necessary in May, as soon as the flower buds can be seen, to remove with the fingers (or carefully with a knife) some of the surplus shoots, especially those which are blind (that is without flower buds at the termination of the shoot), leaving only those which are most promising and well placed. 
INSTRUCTION 8.

\section{TEAS AND HYBRID TEAS}

With a habit of growth spreading rather than upright.

\section{Require moderate pruning.}

For time of pruning see page 88.

For Garden Purposes.-Follow generally the lines laid down in Instruction 4. But, the habit of growth being more horizontal, there is room to leave more stems without overcrowding than in plants of more upright growth. 


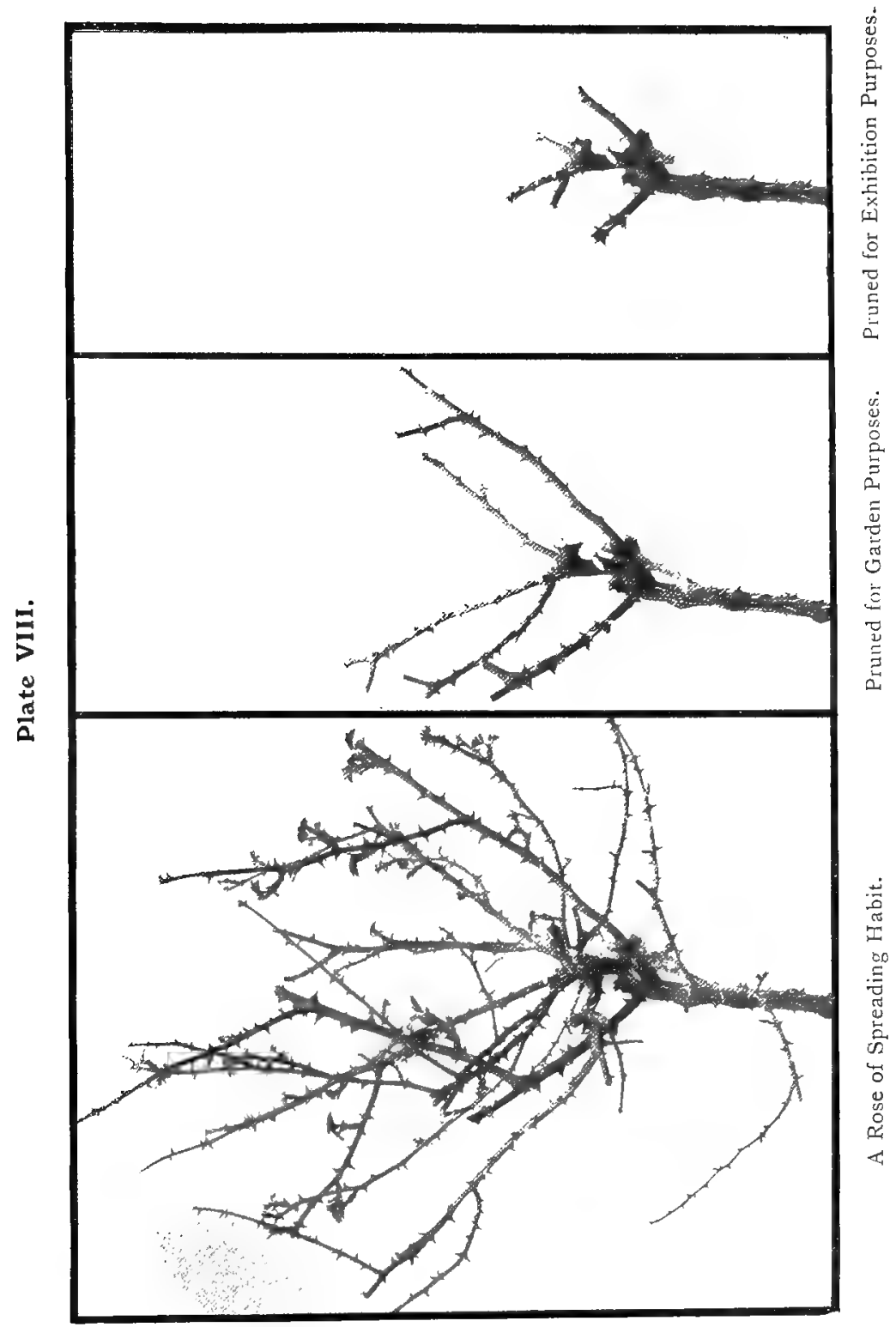





\section{INSTRUCTION 9 .}

\section{TEAS AND HYBRID TEAS}

With a habit of growth spreading rather than upright.

\section{Require moderate pruning.}

$$
\text { For time of pruning sse page } 88 .
$$

For Exhibition Purposes.-Follow generally the lines laid down in Instruction 5. But, the habit of growth being more horizontal, there is room to leave more stems than in plants of more upright growth, without overcrowding. 


\title{
INSTRUCTION Io.
}

\section{HYBRID PERPETUALS, HYBRID TEAS, AND TEAS.}

\section{Climbing Roses.}

\author{
For time of pruning see page 88.
}

All the Roses in this section require very little pruning, and with a few exceptions may be left pretty much to their natural habit of growth. Merely thin out the dead wood and sufficient of the shoots which are more than two years old to keep the shoots from becoming too crowded. The removal of such of the older shoots as may not be required may well be done directly after the plants have flowered in the Summer, the strong young shoots being lightly tied in to take their place. Some of the new growths, if crowded, may also be removed, and strong shoots which are misplaced should either be tied in to fill up blank spaces or cut clean out if not needed at all. The base of the plant frequently becomes bare after a time; this may be obviated by a careful manipulation of the shoots of the past year's growth--bending them down and tying them in to cover the bare part, or by shortening one or two of the shoots coming up from the base. 


\section{INSTRUCTION II.}

\section{PROY ENCE ROSES.-Prune in February or March.}

Remove the old wood and thin out some of the older shoots where at all crowded. Vigorous young base shoots will be found, which, with the best laterals of the twoyear-old wood, should be shortened to some four or six "eyes," leaving the plant a bush from 18 -in. to 24 -in. high.

\section{INSTRUCTION I2.}

\section{MOSS ROSES.-Prune in February or March.}

These may be pruned almost exactly as the Provence, Instruction I I, with some exceptions. (a) The stronger growers should be left somewhat taller. (b) The perpetual Mosses taking more after the Hybrid Perpetuals, may be pruned after the manner described in Instruction 4 for Decorative H.P.'s.

(a)

Blanche Moreau.

Common Moss.

Laneii.

White Bath. (b)

Mme. Edouard Ory. Perpetual White.

Salêt.

\section{INSTRUCTION I3.}

\section{MINIATURE PROVENCE.-Prune in February or March.}

Thin well out and prune to within five or six inches of the ground, only taking care that the plant shall have a certain symmetry when in bloom. 


\section{INSTRUCTION I4.}

\section{FRENCH AND DAMASK ROSES.-Prune early in March.}

The list of these given on page 75 really comprises three classes, but for pruning purposes can be treated as two. There is (a) the Lady Curzon type, (b) the Rosa Mundi type. (a) This is the taller section, and the varieties in it may be treated as tall bushes or even as 5 -ft. to 6-ft. pillars. Thin out the shoots, keeping the best one and two-year-old wood and the best laterals.

(b) Prune the dwarfer growers similarly, but so as to leave the bushes not more than $2 \frac{1}{2}-\mathrm{ft}$. or 3 -ft. high.

$$
\text { (a) }
$$

Lady Curzon.

Mrs. O. G. Orpen. (b)

Rosa Mundi. Red Damask.

\section{INSTRUCTION 15 .}

ROSA ALBA.-Prune early in March.

These may be grown as 5 -ft. to 6 -ft. bushes or bushy pillars. Always thin out the weak wood; the best blooms will be produced by laterals on old shoots. These laterals should be left 9-in. to I2-in. long.

\section{INSTRUCTION 16.}

\section{THE HYBRID CHINAS.-Prune early in March.}

The Hybrid Chinas make some of our best large bushes or bushy pillars, and the shoots may be left about 5-ft. to 6-ft. long. The laterals on the three-year-old wood should be shortened to three or four " eyes," and some of the old wood should be removed. Blairii No. 2 will not require much shortening, and very little thinning.

Blairii No. 2.

Charles Lawson.

Coupe d'Hébé.
Fulgens.

Mme. Plantier. 


\section{INSTRUCTION I7.}

\section{THE HYBRID BOURBONS.-Prune in March.}

These should be left as 4 -ft. to 5 -ft. bushes, and, like the true Bourbons, they bloom best as older plants, so that a good many of the two and three-year-old shoots, with their laterals, may be left with the best of the young base shoots, and all laterals should be left moderately long. (See also Instruction 40.)

\section{INSTRUCTION I8.}

\section{THE HYBRID NOISETTES AND HYBRID MUSKS.-Prune in March.}

The Roses in this section require but little pruning. Thin out only some of the older wood, and tie in the young vigorous shoots where most required to fill any vacant space in the plant. (See also Instruction 40.)

\section{INSTRUCTION 19.}

AUSTRIAN BRIARS (Rosa lutea).-Prune in March.

Here we have to look two years ahead. These briars vary very much in vigour according to the nature of the soil, but they are generally best as bushes from 4-ft. to 6-ft. high. Only the dead wood should be cut out. The strong one-year and two-year shoots from the base should be left, together with the three-year lateral bearing wood, on which much of the bloom is produced. 


\section{INSTRUCTION 20.}

\section{PERNETIANA.-Prune in March.}

This new race of Roses was first obtained by crossing Soleil d'Or with a H.T., and are really hybrids of Austrian Briar (Rosa lutea); but the Council of the National Rose Society have decided that they must now be separately classified, and have given them the name of Pernetiana (R. Pernetiana). They require the same pruning treatment as the Hybrid Teas, but as their growths are somewhat pithy and very liable to be damaged by frost, care must be taken when pruning to see that the cut is made well below the damaged part.

\section{INSTRUCTION 2 I.}

\section{SCOTCH BRIARS.-Prune in March.}

The Scotch Briars may be left about $3-\mathrm{ft}$. high or a little higher. The young suckers, which are freely produced, may be cut shorter, so as to clothe the base. Remove only the old worn out and weak wood; in fact, hardly any pruning at all is required. 


\section{INSTRUCTION 22.}

SWEET BRIARS.-Prune early in March.

Sweet Briars proper (a) should be left about 4 -ft. high. All weak wood and the old naked shoots should be cut away. Penzance Briars $(b)$ are far more vigorous and may be left much taller-from 6-ft. to Io-ft. They throw up long massive base shoots, which may be left to the height required, and those of last year, which will have produced long laterals, should be reduced considerably in height and the remaining laterals left long. Lord and Lady Penzance are less vigorous, and should be left about 6-ft. high. Some thinning will generally be desirable. Used for hedges they must be cut shorter and the long shoots laid down horizontally to keep the base of hedge clothed. (See also Instruction 40.)

(a)

Common Sweet Briar. Hebe's Lip. (b)

Janet's Pride.

Penzance Briarts.

\section{INSTRUCTION 23.}

AYRSHIRES. - Prune in February.

The Ayrshires form a very strong growing section. They require no pruning proper, but should be thinned out sparingly and the dead wood removed.

\section{INSTRUCTION 24.}

\section{SEMPERYIRENS (The Exergreen Rose).-Prue in} February.

The Sempervirens should be treated almost exactly as the Ayrshires (Instruction 23). They are, however, more pendulous and the shoots more slender, so that the laterals may be slightly shortened. These are very good Roses for banks, where they may be left to ramble almost untouched. 


\section{INSTRUCTION 25 .}

THE BOURSA ULTS.-Prune in February.

The Boursaults are huge growers, throwing up bold base shoots which are very rigid. The shoots of the previous year will have produced very strong laterals at regular intervals. They will require no thinning, but otherwise may be treated as the Penzance Briars and the shoots left 6-ft. to Io-ft. long (Instruction 22); alpina flore pleno, a dwarfer grower, belongs to this division and should be grown as a broad, bold bush.

INSTRUCTION 26.

THE BANKSIANS.-Prune in April.

The Banksians do not require pruning, except in the case of old plants, which it will be sometimes necessary to cut back a bit hard to restore vigour. Grown on a wall, the main shoots should be distributed at intervals and the laterals tied in, as it is from these laterals that the bloom will be produced on little 2 -in. flower stems the third year. So do not prune at all for the first three years.

\section{INSTRUCTION 27.}

MULTIFLORA RAMBLERS.-Prune as soon as possible after flowering ceases, and in early spring.

The Ramblers, derived from R. multiflora, date their importance from the introduction of Crimson Rambler. Typically they are rampant growers, throwing out, every year, long, bold shoots both from the base, and from various parts of the stems of previous years. It is from these fresh shoots that the flowering laterals will spring in the following season. 
The plant must be well thinned as early as possible in the autumn. Retain only the very best first and second year wood. The first year shoots may be left long or shortened as the appearance of the plant requires. The tendency of the plant to become bare at the base may be counteracted by shortening some of the lesser stems to induce the lower buds to break. On the second year stems only sound and strong laterals should be retained, and they may be left long or shortened as required. The shortening should be done in the early spring.

Varieties which are the result of hybridising with Wichuraiana roses have the characteristics of that group, and should be treated as advised in Instruction 37 .

The perpetual flowering varieties like Trier are less. rampant in growth and require little more than the removal of old, unripened and unnecessary shoots. This should be done in the winter or early spring.

\section{INSTRUCTION 28.}

\section{SPECIES AND THEIR VARIETIES.-Prune in February or March.}

These need, as a rule, no pruning beyond the removal each year of some of the dead wood. The strong young shoots from the base of the plants will sometimes require shortening back to well-ripened wood, as their tips are apt to die back after the winter frosts. Where any Rose requires exceptional treatment instructions are given after the name of it.

alpina. Treat as a 4 -ft. to 5 -ft. bush. Tip the strong shoots and shorten the laterals and weaker base shoots. It will not require much thinning.

alpina pyrenaica. A dwarf growing variety, with thorns. Treat in the same way as alpina. 
altaica. As a bush 5 -ft. to 6 -ft. The base shoots are numerous and should be pruned to various lengths, and nearly all the older wood can be cut away. Makes a good hedge.

\section{Banksiæ. (See Instruction 26.)}

blanda flore-pleno. Leave the one-year shoots long and shorten the laterals.

bracteata (The Macartney Rose). Train on a warm wall, about 4 -ft. to 5 -ft. high, laying the laterals in. Requires protection in winter and little if any pruning.

canina complicata. Erect growing bush 4 -ft. to 5 -ft. high.

humilis. Dwarf habit. May be treated like R. altaica.

indica. The original China Rose.

lucida. Do not prune except to keep the plant in shape.

lucida flore-pleno. Treat as lucida.

lutea. (See Instruction r9.)

moschata (including Brunonii, Brunonis himalayica). An immense grower, so let it ramble over a summer house or tall arch. There will always be some dead wood to cut away. The bold massive laterals are not numerous, and may be left from 9 -in. to 2 -ft. long.

moschata alba. Leave the one year shoots long, shortening laterals on two-year-old wood.

multiflora. (See Instruction 27.)

nitida. There are two forms, of which the dwarf is best. Makes an effective dwarf hedge, or edging. Requires the removal of old wood but little pruning. The best way to grow it is to peg down the young shoots and cut out the old annually. 
Nuttaliana. Makes a fine bush, which requires thinning out only.

\section{pimpinellifolia. (See Instruction 2I.)}

pomifera. As a 5-ft. to 6-ft. bush. Blooms upon the laterals, which may be left 6 -in. long.

rubrifolia. As a 4 -ft. to 5 -ft. bush. Treat otherwise as alpina.

setigera. Best allowed to ramble over a low tree or wall. It will not require much pruning, except for the removal of old wood; or grow it as a loose wild pillar.

wichuraiana. (See Instruction 37.)

xanthina. Does well pegged down, otherwise treat as an Austrian Briar. (See Instructions 19 and 39.)

\section{INSTRUCTION 29.}

HYBRIDS OF SPECIES.-Prune in February or March. Duponti (gallica $x$ moschata). Treat in the same way as moschata alba. (See Instruction 28.)

humilis $x$ rugosa. A perpetual flowering bush. Should be kept in shape only and occasionally thinned.

macrantha. See French and Damask (Instruction I4).

moschata $x$ multiflora. A high-growing variety. This is sometimes known as polyantha grandiflora. (See Instruction 27.)

moyesii. May be pruned to about 4 -ft. or allowed to grow if space permits, taking out old wood from time to time.

omissa. Vigorous growth. Treat as Instruction $r_{4}(a)$.

sinica Anemone (sinica $x$ indica). Do not prune beyond cutting away dead wood, only train.

Una. A Hybrid from canina. Best as a 5-ft. to 8-ft. loosely-grown pillar. Leave the laterals 9 -in. to I5-in. long. 


\section{INSTRUCTION 30.}

\section{BOURBONS.-Prune in March.}

These bloom mainly on laterals from the old wood, so that a good deal of the best lateral-bearing wood should be left in. They make fine bushes and bushy standards. Do not prune back too hard, but thin well.

(a) Hermosa, also known as Armosa. A variety with all the freedom of the Chinas, to be grown as a low spreading bush, some 2-ft. high. Fellenberg (China) may be pruned much in this way, or it will make a good medium pillar.

(b) Large flowering kinds, making bold fine bushes, very fine standards, and excellent for pegging down. They should be pruned much in the way of the taller of the previous year, and should be well thinned of all weak and cankered shoots, whether they be new or old, as garden H.P.'s (see Instruction 6), keeping Malmaison rather dwarfer.

(a)

Hermosa or Armosa. (b)

Mme. Isaac Pereire.

Mrs. Paul.

Souvenir de la Malmaison.

\section{INSTRUCTION 3 I.}

CHIN AS. - Prune end of March or April.

Chinas must be thinned rather than hard pruned, but some of the stronger shoots may be cut back to encourage new growth from the base of the plant. Another way is to thin out the thin growths after the summer flowering, and a third to cut them closely down in April, when they will flower well but somewhat later. 


\section{INSTRUCTION 32.}

\section{NOISETTES.-Prune in March.}

The Noisettes are mostly moderate climbers, and when used as pillar Roses need to be gradually built up to the required size. It will be necessary to cut away some of the wood which is unsound. As the flowers are produced upon well-ripened laterals, all the healthy old wood must be retained.

(a) Kinds of which the laterals may be fairly closely pruned, and which make 5 -ft. to 6 -ft. pillars, and also make good beds, when pegged down, and large bold standards.

(b) Stronger growers for tall arches and throwing up sturdy base shoots, 7 -ft. to Io-ft., and which also make bold standards.

Lamarque requires a wall. Fortune's Yellow (tender) blooms only once. This variety also requires a wall. In the colder parts of the country it is best under glass. Ophirie and Rêve d'Or should be pruned as little as possible. Maréchal Niel blooms on the shoots. 'The best last year's wood should be retained and left as long as possible. (See also Instruction 40.)

(a)

Aimée Vibert (dwf. perpetual.) William Allen Richardson. (b)

Aimée Vibert (climbing).

A. S. Gray.

Céline Forestier. 


\section{INSTRUCTION 33 . \\ DIJON TEAS.-Pryme in March.}

These varieties of the Gloire de Dijon type make fine large-headed standards, bold massive bushes and good arch and wall Roses. The strongest shoots from the base may be left 6 -ft. to 7 -ft. long, others less strong 4 -ft. to 5 -ft., and some 2 -ft. to 3 -ft., so as to clothe the base. The occasional laterals can be left from 9 -in. to 2 -ft. long according to need. (See also Instruction 40.)

\section{INSTRUCTION 34 . \\ POLYANTHÁS (Pompon).-Prune in March.}

Little dwarf miniature Roses. They will be found to consist of dwarf bushy growths, surmounted by bold flower stalks. All the pruning that is really necessary is to cut away these old flower stems and thin out some of the old wood, when they will break again from the top "eyes" and from the base. When used in small beds they may be cut over close to the ground. The climbing varieties require merely the old dead wood cut out and sufficient of the shoots that are more than two years old removed to keep the plant from becoming too crowded.

\section{INSTRUCTION 35 .}

RUGOSA. - Prune in February.

These throw up numerous suckers from the base; a few of the best of these may be retained about 4 -ft. long, so as to form a bold bush. They will flower and grow freely from the heads of these shoots, and will always break away from the base. An excellent way of growing them is to cut them down each year almost to the ground, then they bloom rather late, but very freely. 


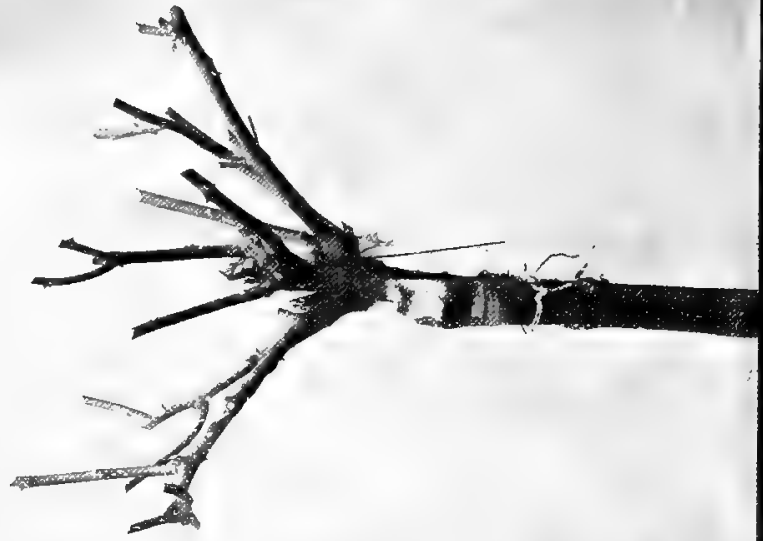

$x$
0
0
0 

Conrad F. Meyer. This is perhaps best as a tall pyramidal bush, some 6-ft. to 9 -ft. high. It makes a splendid pillar, but its Dijon blood betrays itself in a tendency to bareness towards the base which must be counteracted in pruning. In the case of Hybrid rugosas budded on rugosa or other stocks, all suckers below the graft must be suppressed. (See Instruction 33.)

INSTRUCTION 36.

\section{PERPETUAL SCOTCH.-Prune in March.}

This is undoubtedly best grown as a bold halfstandard or tall bush, as it is slightly pendulous in habit. It matters little how it is pruned, except for the sake of symmetry, since it will always flower.

\section{INSTRUCTION 37 .}

\section{WICHURAIANA RAMBLERS (Rosa Wichuraiana).} Prune in the Autumn.

Rosa wichuraiana is a species of rampant trailing growth. It sends up from the base every year a number of strong shoots often Io to I 5 - $\mathrm{ft}$. in length. Established plants also send similar long shoots (continuing laterals) from the long shoots of previous years, and these may be given off at any distance from the base.

The wichuraiana ramblers are hybrids of this species, and are mostly similar in habit of growth, though some of them have rigid upright stems in contrast with the trailers of the parent species. As the flowering laterals of next season will spring from the long shoots just formed, it is essential that these long shoots should be preserved when pruning. The pruning should be done in the early autumn, or the flowering laterals are liable to be damaged, as they begin to start into growth very early in the year. 
If a plant of moderate size is wanted, all the old wood should be cut right away to the base, so that the plant is left with only the long basal shoots of the last season's growth. These may be shortened or left full length as desired; if overcrowded some of them may be removed to the base. If the number of these basal shoots is deficient they may be supplemented by leaving some of the older shoots which carry long continuing laterals and bending back these laterals to furnish the parts deficient.

If a large plant is required (as for a pergola, for covering a wall or for festoons on ropes.or chains), sufficient of the new basal shoots should be left to furnish the lower part of the plant, and the older stems which carry long continuing laterals should be left with these laterals to furnish the more distant parts; but from these old stems all the small spindly laterals should be cut right away. All the old stems which do not carry long continuing laterals should be cut right away to the base.

\section{WICHURAIANA POMPONS.}

The wichuraiana pompons should be treated in the same manner as the polyantha pompons. (See Instruction 34.)

\section{INSTRUCTION 38. \\ STANDARD ROSES.}

For general garden purposes only strong growing varieties should be employed.

(a) Such sorts as Gloire de Dijon, Bouquet d'Or and W. A. Richardson will require judicious thinning, i.e., removal of worn out or superfluous shoots, preserving the long well-ripened shoots made the previous season 


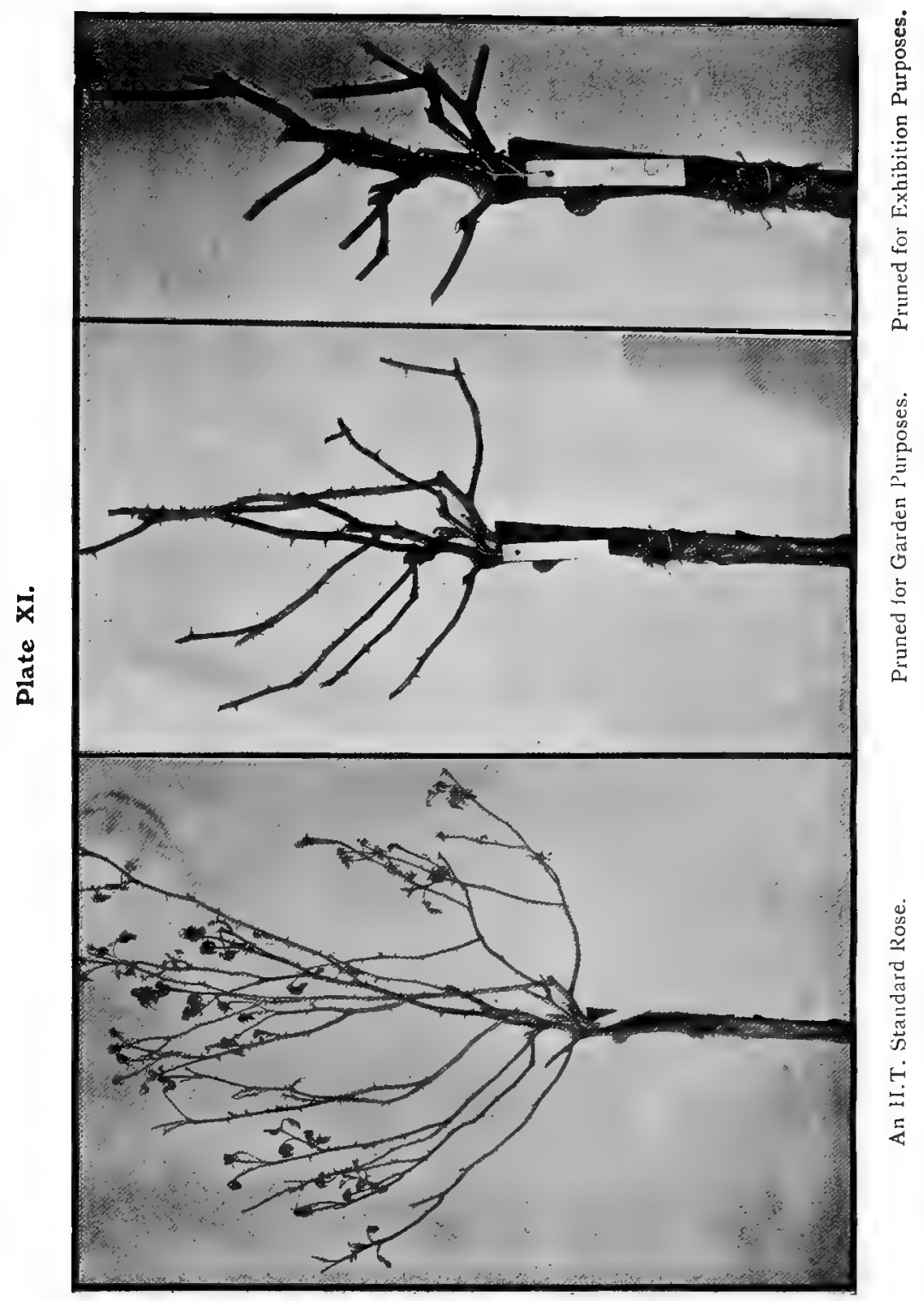


for almost their entire length. These may be shortened slightly, as unripened tips are often damaged by frost, but on no account cut hard back, or the result will merely be the formation of long flowerless shoots.

(b) Standard H.P.'s and H.T.'s. (See Plates ro and II.) These may, generally speaking, be pruned in accordance with the instructions given for dwarf plants. It is important to have regard to the formation of a shapely head and to keep the centre of the plant well open. On Standards it will be observed that fresh shoots from the point of union of the Rose with the stock are not so frequently made as in dwarf plants. The pruning will therefore, as a rule, mean cutting back the shoots made the previous season, nearly to last year's point of pruning. If left long the head will soon become unshapely.

The weaker growing Teas will require severe pruning, and with this class no harm is done in cutting back almost to the union of the Rose with the stock. In all cases weak unripe or frosted shoots should be entirely removed.

\section{Weeping Standard Roses.-Prune in Autumn.}

(c) When the plant is well supplied with long strong shoots of current year's growth (see illustrations), remove all the older shoots as near to the head of the stock as possible. It may be necessary to leave a few of the older shoots to preserve the balance of the plant, but from them cut away the old twiggy flower stems. These old stems will produce foliage if not many flowers. If the new strong shoots are very numerous some of them may be removed to prevent overcrowding. 
When the shoots are so long as to trail on the ground they may be shortened, as they are likely to be injured by strong winds. See illustrations.

\section{INSTRUCTION 39.}

\section{ROSGS FOR PERGOLAS AND PILLARS.}

A pergola has been well described as " that pleasant shape of covered way that we have borrowed from the Italians."

This covered way is a series of linked arches, more or less roughly roofed in, on which ramble all kinds of climbing plants. It should be built substantially, or, just when the plants are attaining their full size, the supports may begin to give, and much of the work will have to be done again.

The pergola should serve a definite purpose. Properly it should lead from one point to another, and where possible this should be kept in view. A pergola is sometimes useful in providing a screen, or to form the boundary between the Flower Garden proper and a rougher section of the garden or paddock.

It is of the first necessity that a pergola should be well clothed, and it is to ensure this result that suitable Roses should be selected and a proper system of pruning adopted.

Among the larger growing climbing Roses are a select few which are particularly fitted for this purpose. These are kinds which, whilst rambling far and wide, will clothe the sides as well as, the top of the arches. They should be floriferous and of luxuriant foliage, such as the Ayrshires and Sempervirens. Amongst the most perfect pergola Roses are the wichuraiana 


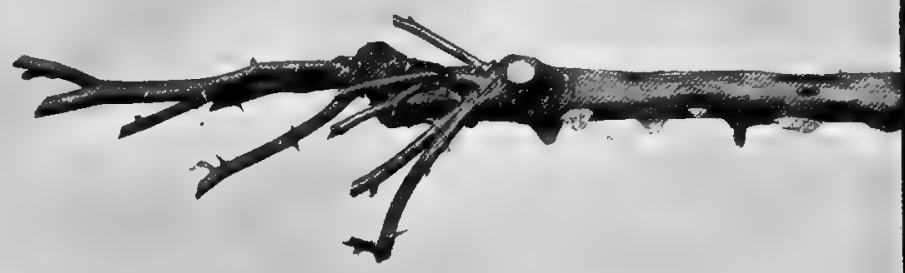

莕

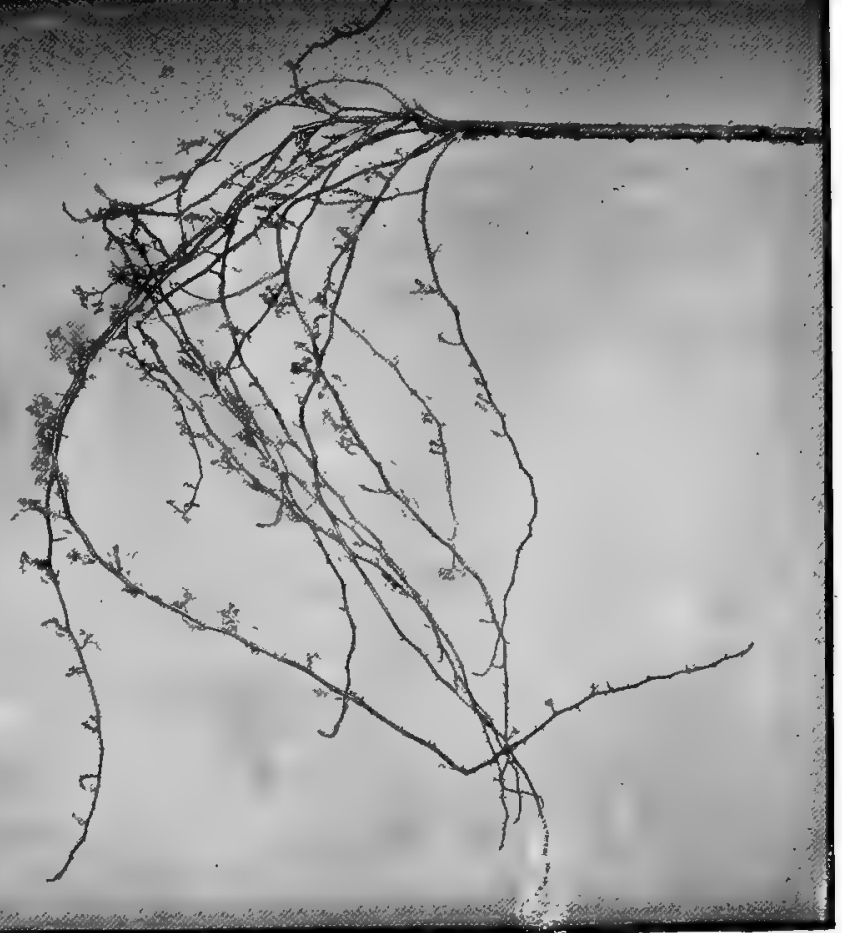

व 



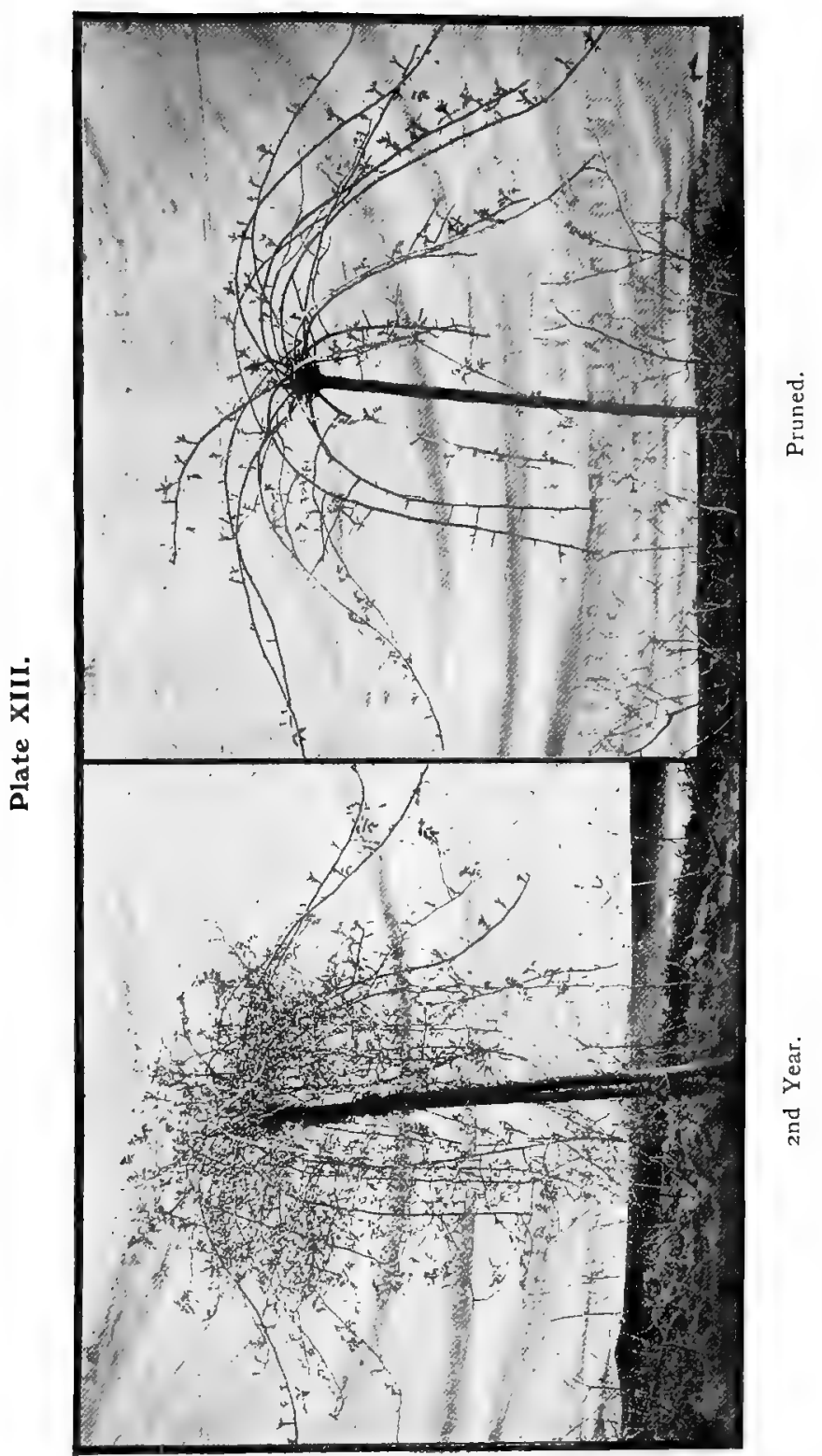





\section{Plate Xiv.}

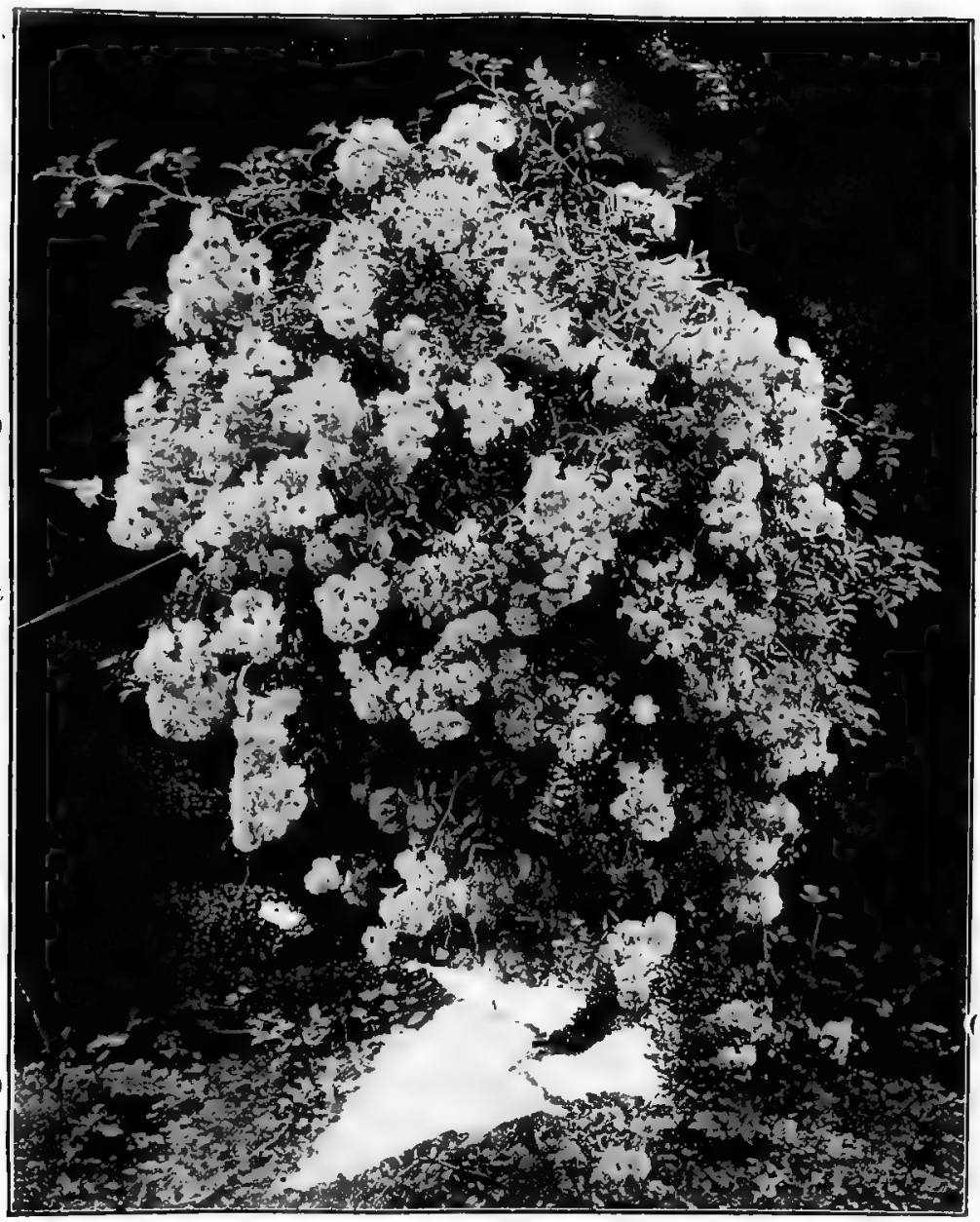

Third year, perfection.

Photographed in Mr. E. F. Hicks' Nursery, Hurst, Twyford. 

and multiflora ramblers. These have close, almost evergreen foliage, and produce, on long pendulous shoots, numerous bunches of highly coloured and fragrant flowers. Some are perpetual; all bloom over a period of some weeks; they break continually from the base, and the laterals hang down with the abandon necessary to a pergola.

As to the treatment of Roses grown in this way:-

The first point is to clothe the pergola as quickly as possible; therefore, rapid growing varieties should be chosen, which may afterwards be supplemented, when necessary, as recommended above. During the spring, after planting, the branches should be shortened according to the size of the plant and the effects of the previous winter; the second year thin out carefully, retaining the best shoots of the previous year for the sake of the blooms and the best and strongest baseshoots to reach overhead. When thoroughly established, the plants should be pruned in the early autumn as follows:-

Begin by cutting away all inferior or dead wood, care being taken that the shoots left are not too numerous. At the same time it is very important that those shoots which will carry some flower overhead should be retained.

When the pruning is completed and when re-tying the shoots, avoid tying too closely, as air and light are the chief factors in causing the lateral "eyes" to break. Reference to pages 30 to 74 will direct the reader to the treatment for any particular variety, but as a general rule Roses on pergolas will require less pruning, and more training and tying, than is the case with those on 
ordinary pillars. Kinds like the Ayrshires may often be left two or three years practically untouched, when some thinning will become necessary. Flowers and foliage, and plenty of them, are the qualities most to be desired in pergola Roses.

\section{INSTRUCTION 40. \\ EARLX AUTUMN THINNING.}

Most of the climbing and strong growing Roses are much benefited by pruning after their summer flowering. This is best done in the late summer or early autumn, say in August.

The summer flowering varieties should have the wood that has flowered cut right away. The majority of these varieties produce the best blooms and the best display of flowers from wood of the preceding year's growth, or on short laterals from it, and the object of this pruning is to encourage the young growth starting from or near the base of the plant, and give it room to ripen. The strong young shoots so produced may if necessary be tied in to prevent their breaking, but should on no account be shortened or have their growth checked at this period of the year.

Summer Roses of a less vigorous habit, i.e., those which are bushes rather than climbers, may receive similar treatment in a modified form. The Moss Rose may be taken as an example of this class. Here a considerable thinning out of shoots that have flowered is beneficial, but the young growth is less robust, and it is not usually practicable or even desirable to cut out all the shoots that have flowered.

The Briars require rather different treatment. These flower as a rule on the sub-laterals, moreover the shoots 
thrown up from the base are, except in the case of some of the Penzance Briars, less vigorous than those of the summer flowering climbers. A thorough thinning out every three or four years will suffice. It is well not to tie up the young growth in the summer before it has ripened, and Briars should never be pruned in the ordinary sense of the expression, that is by cutting back the shoots.

Perpetual Roses. The strong growing Hybrid Perpetuals, and some of the Hybrid Teas which resemble them in habit of growth, should be looked over, and any old wood that has produced weak and sprayey growth removed, leaving three or four strong shoots in each plant to grow and ripen. The extent to which this late summer thinning should be carried will depend partly on the amount and character of the autumn flowering that is desired, but it is surprising how much judicious thinning out of old wood can be done without materially interfering with the autumn display.

Where any of the Dijon Teas are grown as pillars or bushes a moderate summer thinning out of old wood is beneficial, but on walls and screens it is best to leave them till the spring pruning.

Cultivation with the Dutch hoe should be continued regularly throughout the summer, and in case a drought set in a few copious waterings should be administered. But when done at all watering should be very thorough; a watering that only moistens the upper four or five inches of soil is worse than none at all. After watering stir up the soil to see if your water has got well down, and give an extra hoeing the following day. Liquid manure should not be given after July. 


\section{INSTRUCTION 4 I.}

\section{PEGGED DOWN ROSES.-Prune in March.}

Only strong growing Roses should be employed for pegging down. A few of the longest and ripest shoots, not more than three or four to a plant, are to be retained, cut to the length required, and pegged down carefully as nearly horizontally as possible, and the remainder of the shoots cut right out. In the summer several young shoots from the base of the plant are to be allowed to grow out, and in the following spring the shoots that have flowered are cut right away and a similar number of the young shoots that have grown up are to be pegged down in their place.

Pegged down Roses require extra good cultivation.

The following Roses are recommended for pegging down :-

Frau Karl Druschki.

Gruss an Teplitz.

J. B. Clark.
Mme. Isaac Pereire. Mme. Jules Gravereaux. Zéphirine Drouhin.

\section{INSTRUCTION 42.}

\section{ROSES IN POTS UNDER GLASS.}

Prune at end of December or early in fanuary for blooming about three months later.

It is assumed that the pots have been plunged in a bed of ashes or other material, and standing out of doors in a suitable place all the summer and autumn.

The soil in the pots should be allowed to become fairly dry before the plants are pruned. Some growers prune the plants before taking them in under glass, others house the plants a few days before pruning. 
It is important that the plants should have cool treatment before, and for some time after pruning, so that conditions are made to approximate closely to those prevailing out of doors, say in March, with regard to the outdoor plants. It is only necessary just to exclude frost. A hurried start will mean weak shoots and poor blooms. The first year the plants must be pruned hard back. All sappy, weak and crowded shoots must be removed, and the well ripened ones that remain shortened back to two or three "eyes." In the second year, if the plants have made a sufficient number of well ripened growths from the base, old wood may be removed and these growths shortened to three or four "eyes." In the absence of these new growths from the base the existing shoots should be shortened nearly back to the point of pruning last year. Avoid leaving shoots long, or a straggling and unmanageable plant will be the result, whereas the aim should be to secure a well-balanced and dwarf plant with good shoots as equally distributed round it as possible. To a large extent this will be arranged later, when the thinning out of the growing shoots is done. These instructions apply to the Hybrid Perpetuals, Hybrid Teas, and Teas, and generally the weaker the grower the more severe the pruning may be.

Varieties in other sections must be dealt with on similar lines to those described in Instructions I I to 37 . 


\section{INSTRUCTION 43 .}

\section{CLIMBING ROSES UNDER GLASS.}

Climbing Roses under glass will require treatment according to the class to which they belong.

For instance, Maréchal Niel, Climbing Niphetos, Fortune's Yellow, and W. A. Richardson should be pruned after flowering. The wood which has flowered should be cut hard back to induce the formation of long growths, which will be well ripened during the summer and trained in for flowering the following spring.

Generally the practice will be to remove worn out or superfluous shoots as early as possible and to give all the light and air to a sufficient number of the new shoots, from the laterals of which flowers are expected the following season.

Whether the Climbing Roses are planted out, or in pots, the treatment will be the same, except that those in pots should be plunged in ashes out of doors during the summer and autumn. (See also Instructions $\mathrm{IO}_{2}$ 32, and 33.) 


\section{INSTRUCTION 44.}

\section{HYBRID BRACTEATA.}

This is a new race of Roses, of which that charming variety Mermaid may be given as a typical example. No matter how hard the shoots are cut back the young growths are sure to bloom. The best way to treat them is to thin out the old wood early in the spring and shorten back the young shoots that may be damaged by frosts. The type is best grown as climbers against any wall or building, aspect not material, but south-west wall for preference, or as a pillar or large bush in the open. They are quite hardy, Mermaid having withstood 30 degrees of frost without injury. They grow and flower very freely in the autumn. 


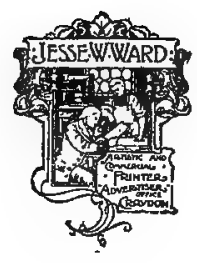





9-

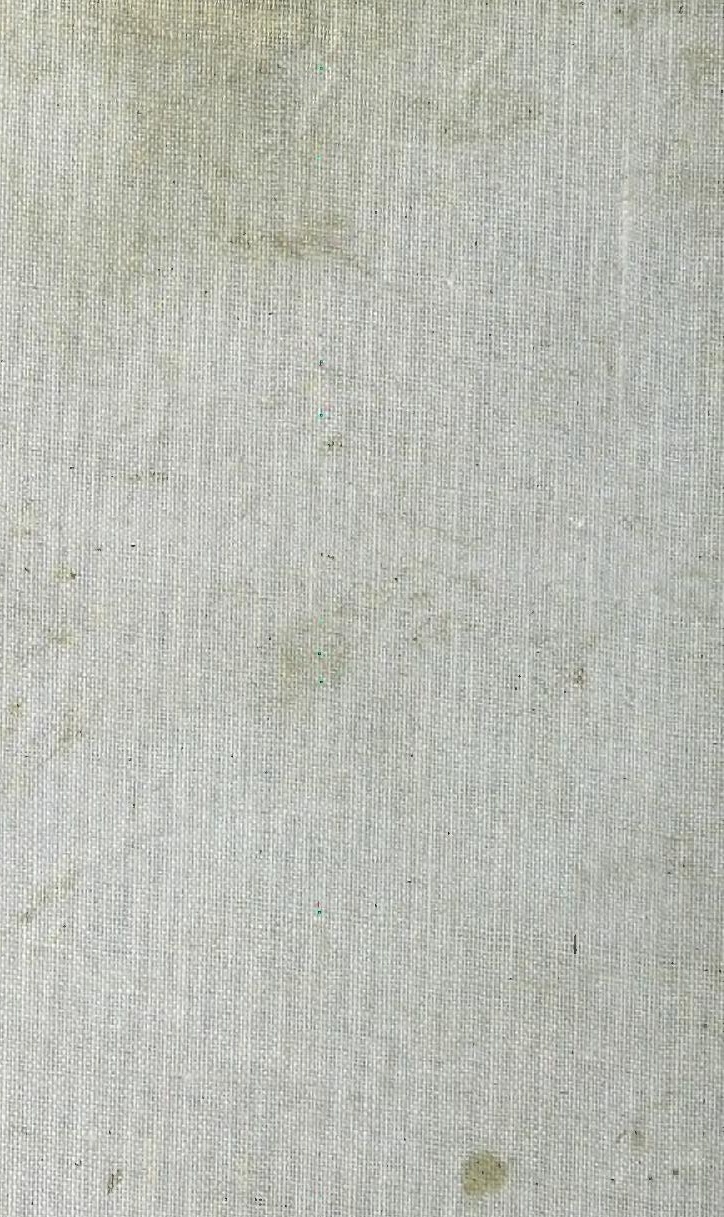

